\title{
ROBOTIC TOOLING CALIBRATION BASED ON LINEAR AND NONLINEAR FORMULATIONS
}

\author{
By \\ Mohamed Helal, B.Eng \\ Aerospace Engineering \\ Ryerson University, 2013
}

A thesis presented to Ryerson University
in partial fulfillment of the requirement for the degree of
Master of Applied Science
in the Program of
Aerospace Engineering

Toronto, Ontario, Canada, 2015

(C) Mohamed Helal, 2015 


\begin{abstract}
AUTHOR'S DECLARATION
I hereby declare that I am the sole author of this thesis. This is a true copy of the thesis, including any required final revisions, as accepted by my examiners.

I authorize Ryerson University to lend this thesis to other institutions or individuals for the purpose of scholarly research.

I further authorize Ryerson University to reproduce this thesis by photocopying or by other means, in total or in part, at the request of other institutions or individuals for the purpose of scholarly research. I understand that my thesis may be made electronically available to the public.
\end{abstract}




\title{
ABSTRACT \\ Robotic Tooling Calibration Based on Linear and Nonlinear Formulations
}

\author{
Mohamed Helal \\ A thesis of the degree of \\ Masters of Applied Science, 2015 \\ Department of Aerospace Engineering, Ryerson University
}

Industrial robot calibration packages, such as ABB CalibWare, are developed only for robot calibration. As a result, the robotic tooling systems designed and fabricated by the user are often calibrated in an ad-hoc fashion. In this thesis, a systematic way for robotic tooling calibration is presented in order to overcome this problem. The idea is to include the tooling system as an extended body in the robot kinematic model, from which two error models are established. The first error model is associated with the robot, while the second error model is associated with the tooling. Once the robot is fully calibrated, the first error will be reduced to the required accuracy. Thus, the method is focused on the second error model. For the tool error calibration, two formulations were used. The first is a linear formulation based on conventional calibration as well as self-calibration methods while the second is a nonlinear formulation. The conventional linear formulation was extensively investigated and implemented while the selfcalibration was proven to be inadequate for the tooling calibration. Moreover, the nonlinear formulation was demonstrated to be very effective and accurate through experimental result. The end-effector position estimation as well as the tool pose estimation were obtained using a 3D vision system as an off-line error measurement technique. 


\section{ACKNOWLEDGEMENTS}

I would like to express my deepest appreciation to my supervisor Dr. Jeff $\mathrm{Xi}$, for his extensive guidance, support and encouragement not only during my master's degree but also during my undergraduate degree. His great knowledge and patience have been really helpful for guiding me towards the right path to solve all the problems I faced. It has been a great pleasure for me to work under his supervision.

I would also like to offer my specials thanks to Mr. Yu Lin for all his help, time and effort during my research period.

In addition, I would like to express my gratitude to all my co-workers at Amel Group especially Ms. Johanna Malisani, Mr. Peter Cresnik and Mr. Khalid Saleh for their help and support.

Last but not least, I would like to thank my family and friends for their support, encouragement and understanding during the past school years. 


\section{TABLE OF CONTENTS}

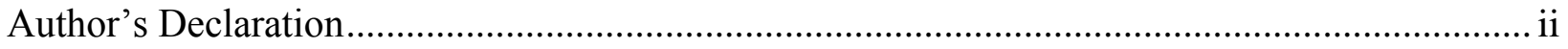

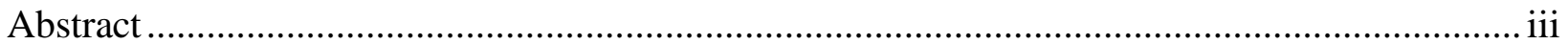

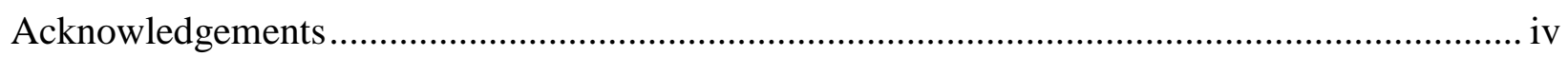

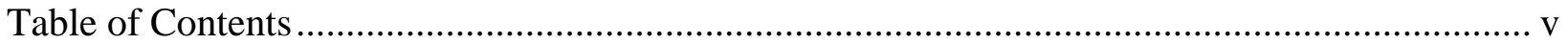

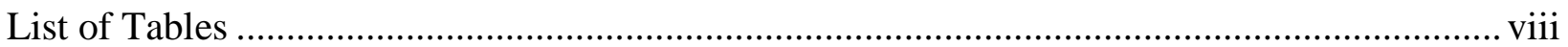

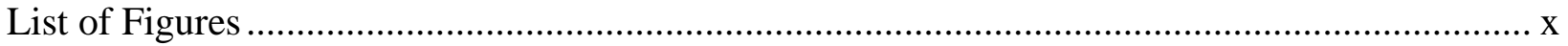

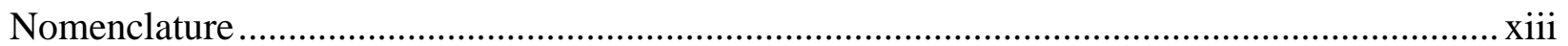

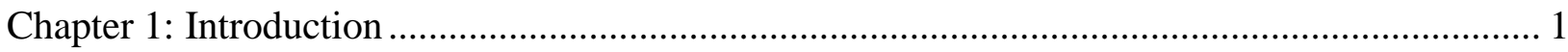

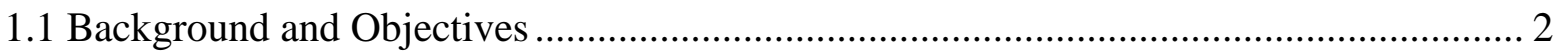

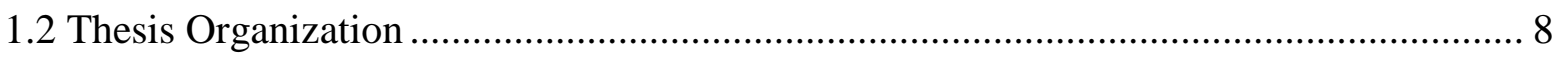

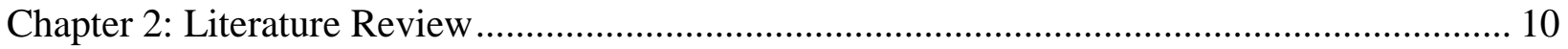

2.1 Kinematic Modeling for Manipulator Calibration......................................................... 11

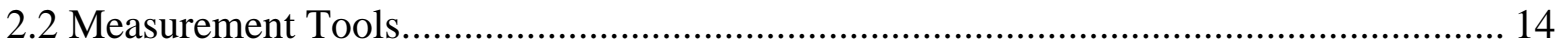

2.2.1 Laser Tracking Systems............................................................................... 16

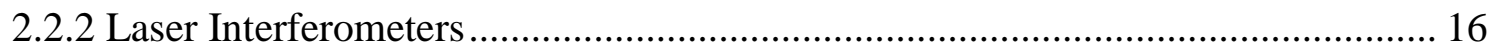

2.2.3 Coordinate Measuring Machines (CMMs) …………........................................ 17

2.2.4 Camera Type Devices........................................................................................ 19

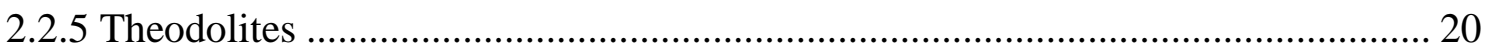

2.2.6 Wire-Draw Encoders .................................................................................... 21

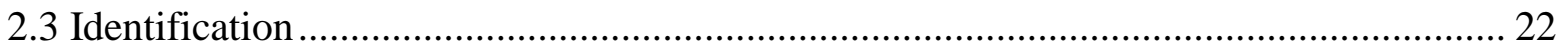

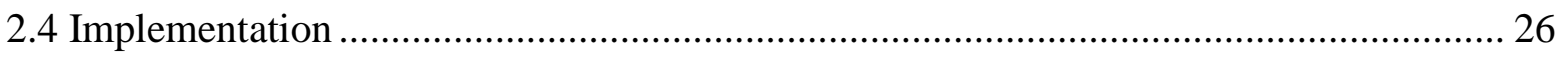

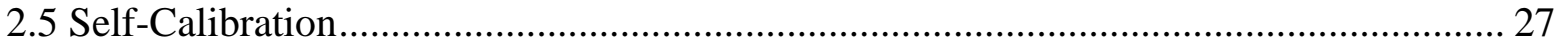




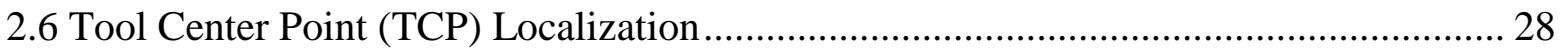

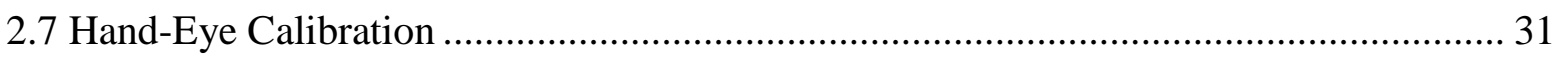

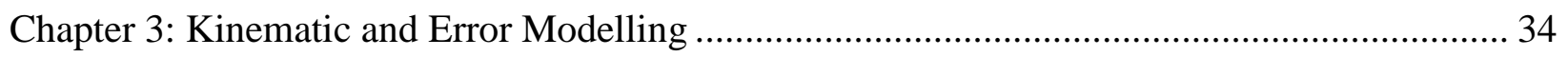

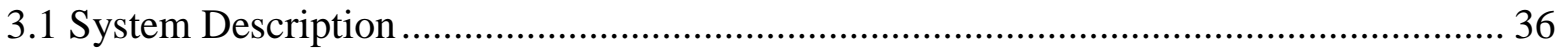

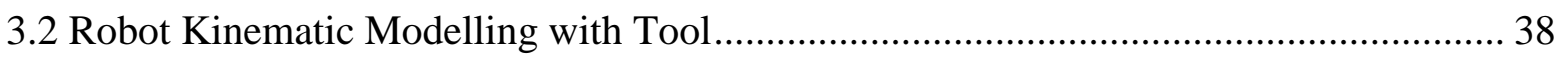

3.2.1 Position and Orientation ................................................................................... 38

3.2.2 Calibration System Kinematic Modeling …………........................................... 40

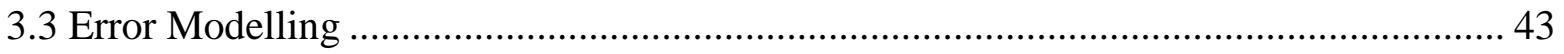

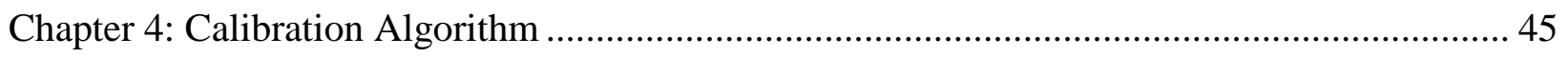

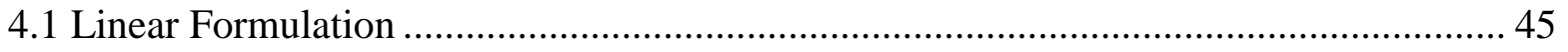

4.1.1 Eye-to-Hand vs. Eye-in-Hand ............................................................................ 47

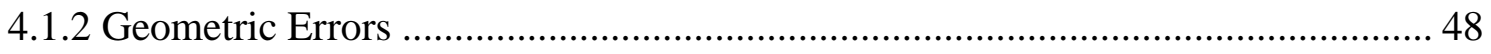

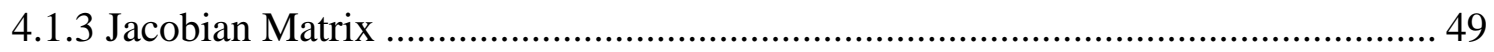

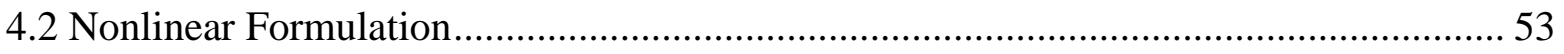

4.2.1 Genetic Algorithm .......................................................................................... 54

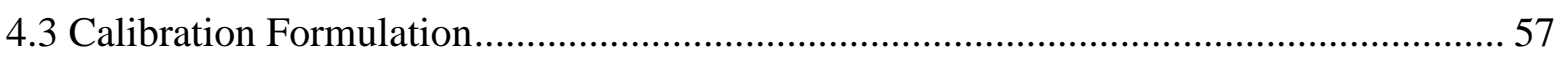

4.4 On-line vs. Off-line Error Measurement........................................................................... 59

4.4.1 On-line Error Measurement for Robots ................................................................ 59

4.4.2 On-line Error Measurement for Robotic Tooling Calibration ................................ 62

4.4.3 Off-line error measurement for Non-linear Tool Calibration formulation ............. 67

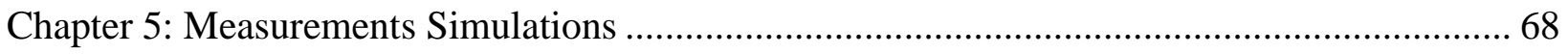

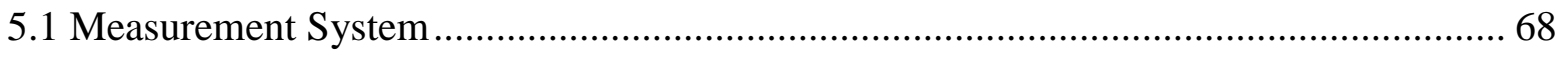

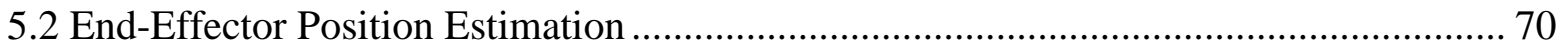




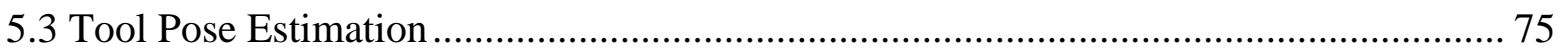

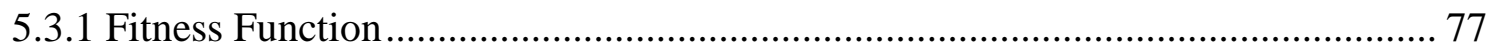

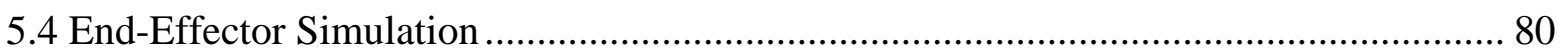

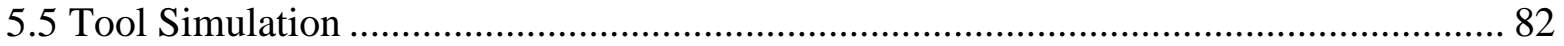

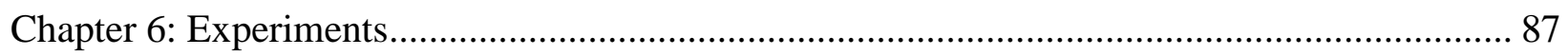

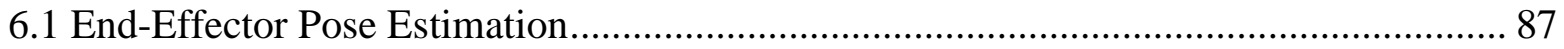

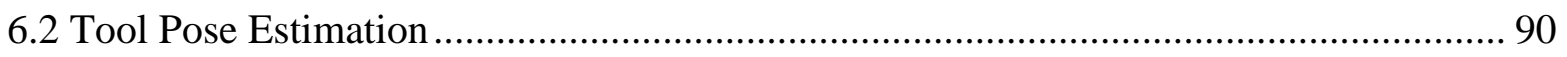

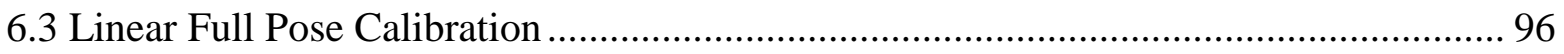

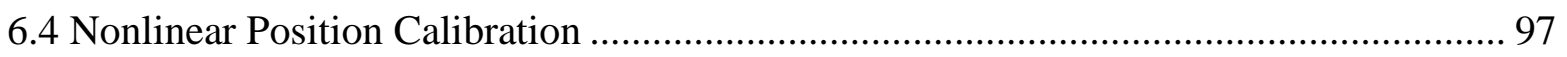

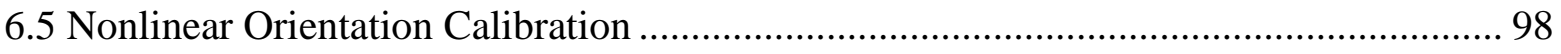

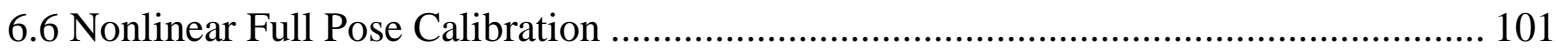

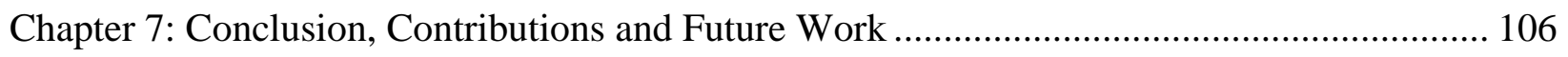

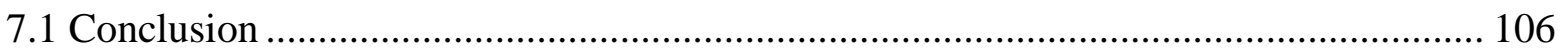

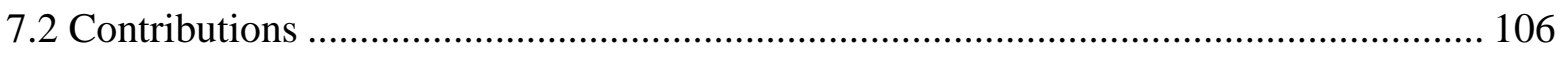

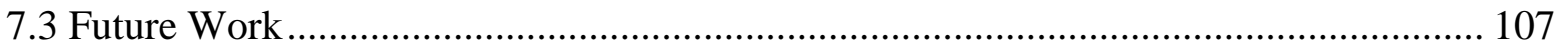

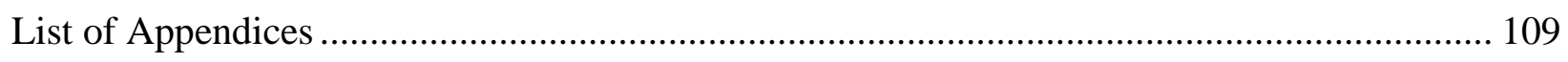

Appendix A - Plane Fitting .............................................................................................. 109

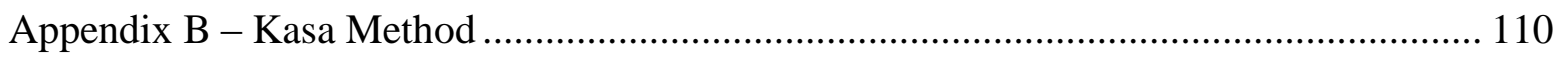

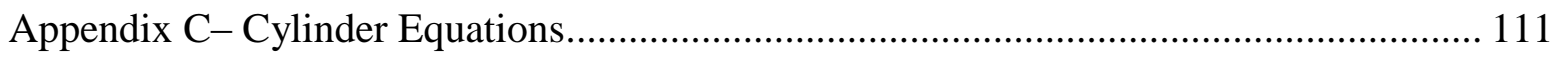

Appendix D - Robot End-Effector in Base Frame............................................................ 113

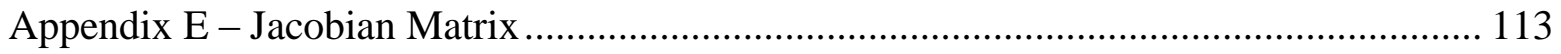

Appendix F - 3D Vision System................................................................................... 114

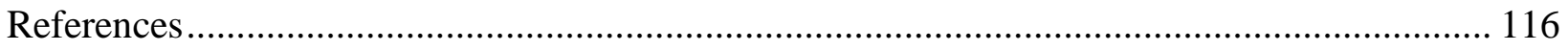




\section{LIST OF TABLES}

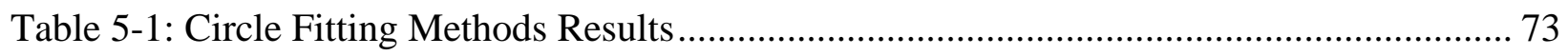

Table 5-2: Simulated Cylinder Data Points Parameters ........................................................ 79

Table 5-3: Estimated GA Cylinder Parameters ............................................................ 80

Table 6-1: End-Effector Location with Respect to the Camera Frame .................................... 88

Table 6-2: Cylinder GA Results with respect to the Intermediate Frame................................. 92

Table 6-3: Monte Carlo Simulation Results ........................................................................ 94

Table 6-4: Measured and Nominal Rotation Matrices and Position Vectors ............................ 95

Table 6-5: Linear Formulation Estimated Geometric Errors ............................................... 97

Table 6-6: Calibrated Tool Pose Rotation Matrix and Position Vector.................................... 97

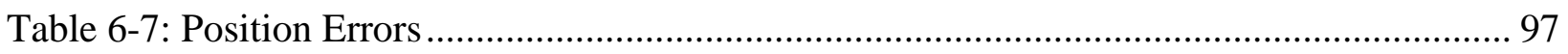

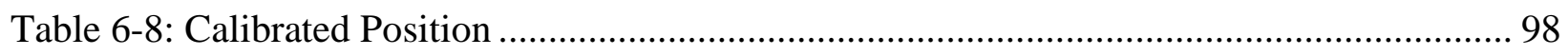

Table 6-9: Orientation Errors for 500 Generations and 100 Population Size........................... 99

Table 6-10: Measured, Nominal and Calibrated Rotation Matrices with respect to the Camera

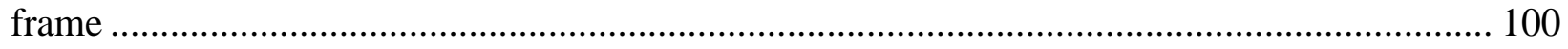

Table 6-11: Errors with 500 Generations and 300 Population Size...................................... 101

Table 6-12: Position and Orientation Errors Based on 500 Generations and 100 Population Size 102

Table 6-13: Calibrated Rotation Matrix and Position Vector Based on 500 Generations and 100

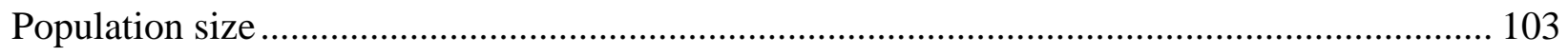

Table 6-14: Position and Orientation Errors with 500 Generations and 300 Population Size ... 103

Table 6-15: Calibrated Rotation Matrix and Position Vector Based on 500 Generations and 300 Population Size .............................................................................................. 104

Table 6-16: Position and Orientation Errors with 500 Generations and 500 Population Size ... 104 
Table 6-17: Calibrated Rotation Matrix and Position Vector Based on 500 Generations and 500

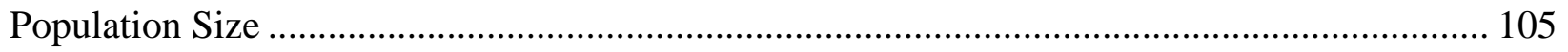

Table A-1: End-Effector Parameters with respect to the Base Frame .................................... 113

Table A-2: Rotation Matrix and Position Vector of the End-Effector with respect to the Base

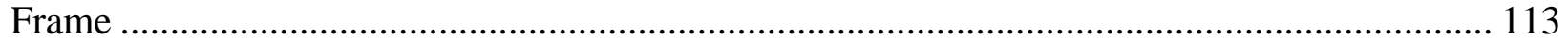




\section{LIST OF FIGURES}

Figure 2-1: Laser Tracking Concept Diagram [5] .............................................................. 16

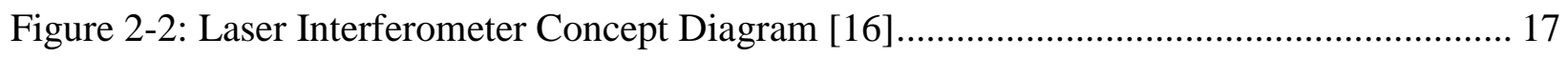

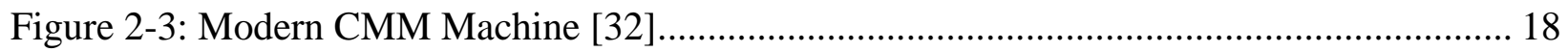

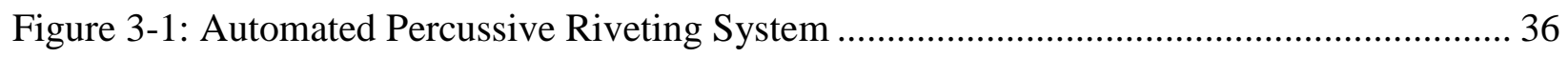

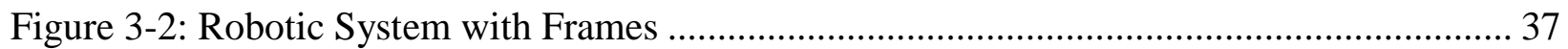

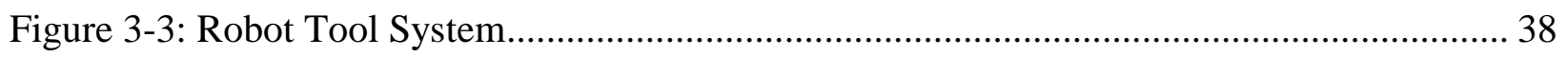

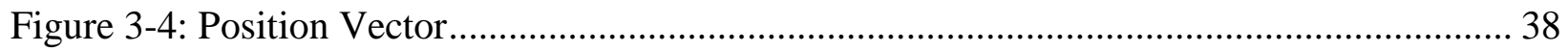

Figure 3-5: Robot - 3D Vision System Diagram ....................................................................... 41

Figure 4-1: Tool - End-Effector Relation ............................................................................ 48

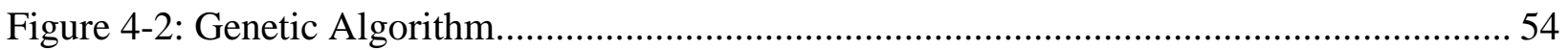

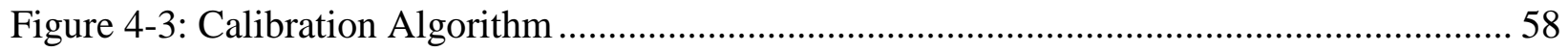

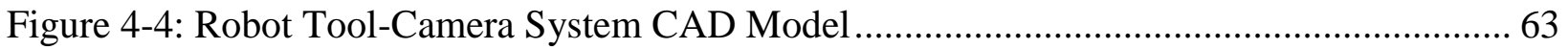

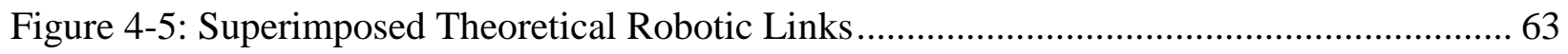

Figure 4-6: Final Theoretical model diagram. ............................................................................ 64

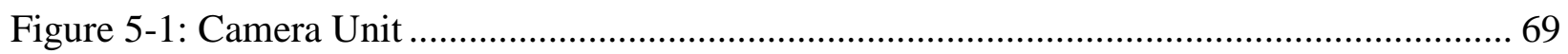

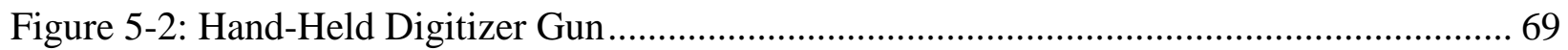

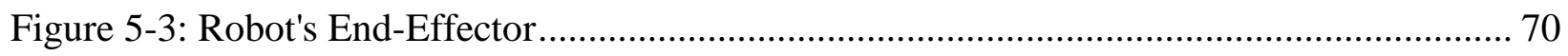

Figure 5-4: Circle Fitting using Kasa Method vs. Least Square Method ..................................... 73

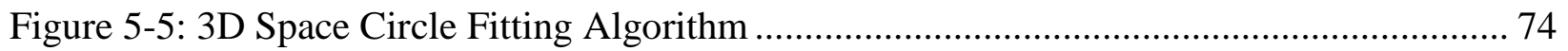

Figure 5-6: Simulated 3D Circle Fitting Algorithm ………...................................................... 75

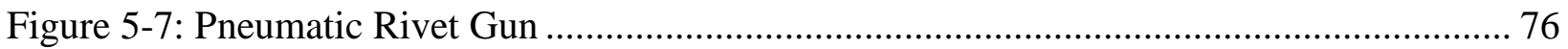

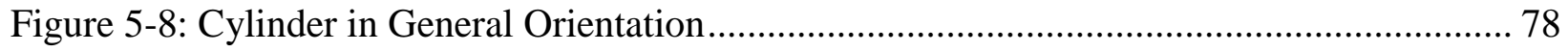


Figure 5-9: Generated Point Cloud and Best Fit Cylinder. 79

Figure 5-10: Cylinder Fitting GA with 500 Generations and 500 Population Size.................... 80

Figure 5-11: Circle Simualtion Data Points.................................................................. 81

Figure 5-12: Circle Center Accuracy vs. Number of Points ................................................ 82

Figure 5-13: Cylinder Point Cloud with Noise ................................................................ 83

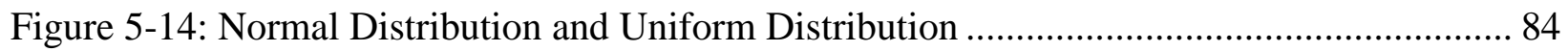

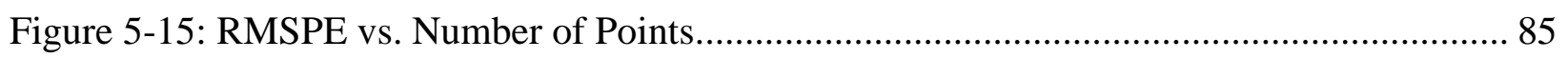

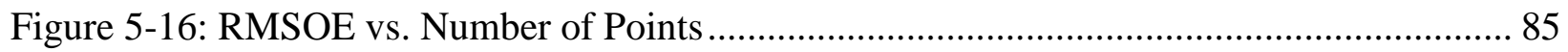

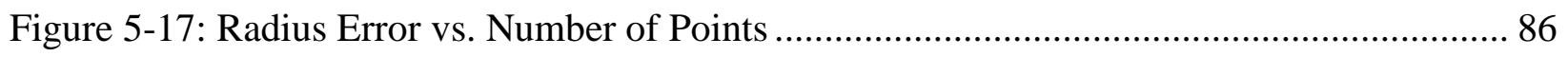

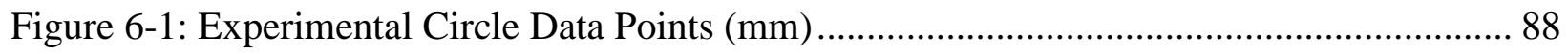

Figure 6-2: End-Effector Center and Frame Determination ............................................. 89

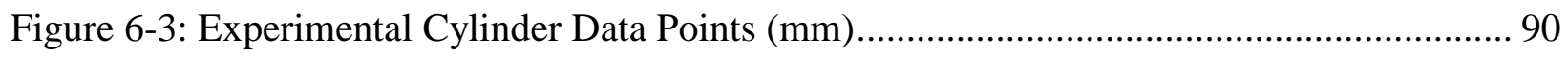

Figure 6-4: Introducing an Intermediate Frame Diagram .............................................. 91

Figure 6-5: Cylinder Fitting GA with 500 Generations and 500 Population Size..................... 93

Figure 6-6: Cylinder Fitting Experimental Point Cloud .................................................... 94

Figure 6-7: Camera, End-Effector and Gun Set-up Diagram ............................................. 95

Figure 6-8: Linear Calibration RMSE vs. Number of Iterations .......................................... 96

Figure 6-9: Position Only Calibration with 500 Generations and 100 Population Size .............. 98

Figure 6-10: Orientation Only Calibration with 500 Generations and 100 Population Size........ 99

Figure 6-11: Orientation Only Calibration with 500 Generations and 300 Population Size...... 101

Figure 6-12: Full Pose Calibration with 500 Generations and 100 Population Size ................. 102

Figure 6-13: Full Pose Calibration with 500 Generations and 300 Population Size ................. 103

Figure 6-14: Full Pose Calibration with 500 Generations and 500 Population Size ................. 104 
Figure A-1: Best Fit Plane Generated for Multiple Points

Figure A-2: Cylinder Rotation Sequence.

112 


\section{NOMENCLATURE}

\section{Symbols}

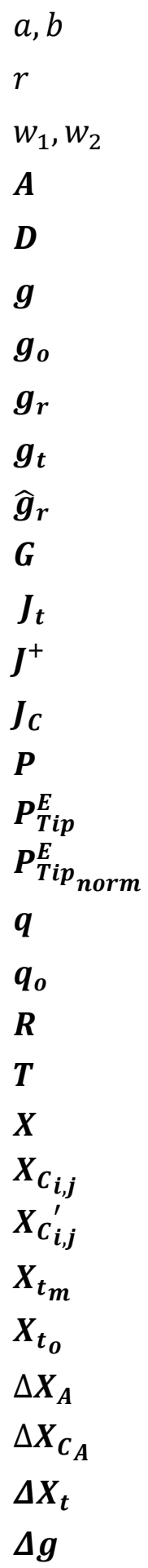

\section{Definition}

Center Location Corresponding to X and Y Axes

Radius

Weighting Factors

Augmented Diagonal Matrix of Rotation Matrices

Self-Calibration Measurements Matrix

Robot Geometric Parameters

Nominal Geometric Parameters

Nominal Robot Geometric Parameters

Nominal Tool Geometric Parameters

Calibrated Robot Geometric Parameters

Self-Calibration Jacobian Matrix

Tool Jacobian Matrix in the Base Frame

Jacobian Pseudo Inverse

Jacobian Matrix for Camera Frame

Position Vector

Tool Length Position Vector

Normalized Tool Length Position Vector

Joint Variables

Nominal Joint Variables

Rotation Matrix

Homogeneous Transformation

Robot Tip Pose

Measured Target Position

Actual Target Position

Measured Tool Tip Pose

Nominal Tool Tip Pose

Augmented Tip Errors Vector

Augmented Tip Errors Vector for Self-Calibration

Robot Tip Pose Errors

Robot Geometric Parameters errors 


$\begin{array}{ll}\Delta \boldsymbol{p} & \text { Robot Joint Variables Compensation } \\ \boldsymbol{\Delta} \boldsymbol{q} & \text { Compensation Errors } \\ \mathfrak{R}_{\boldsymbol{E}}^{\boldsymbol{B}} & \text { Block Diagonal Matrix of Rotation Matrices } \\ \boldsymbol{\theta} & \text { Orientation Angles Vector }\end{array}$

\section{Sub/Superscripts Definition}

$\begin{array}{ll}C & \text { Camera Frame } \\ m & \text { Measured } \\ o & \text { Nominal } \\ R & \text { Robot Frame } \\ r & \text { Robot } \\ T & \text { Tool Frame } \\ t & \text { Tool } \\ T i p & \text { Tool Tip Frame } \\ V & 3 \text { D Vision System Frame }\end{array}$




\section{CHAPTER 1: INTRODUCTION}

Presented in this thesis is a development of a novel method for robotic manipulator tool center point calibration. This chapter is composed of two main sections that provide a full and quick overview of the topics discussed in the following thesis.

The first section of this chapter provides a general overview of the thesis background regarding multiple topics. It starts by a brief history of the industrial automation development during the last few decades. It also provides a complete description of the robotic manipulators and their characteristics as well as their advantages and disadvantages. It progresses by discussing the main disadvantages focusing on the lack of accuracy of its main sources and potential solutions available. Subsequently, an introduction to the calibration process steps is discussed. Calibration process is also introduced from a mathematical point of view as an optimization problem and its possible solutions. The user problem regarding tool center point determination is also explained. Finally, this section ends by listing all the assumptions taken into consideration as well as the objectives and contributions of the thesis.

The second section of this chapter includes a full outline of the thesis and a brief explanation of each of the following chapters. 


\subsection{Background and Objectives}

Major industries such as aerospace and automotive industries are mainly focusing on increasing their productivity rates by increasing the efficiency of their industrial processes. As a result, the main trend is directed towards the automation of most of these processes. Since the use of the first automated robotic system in the early 1960s and until the current date, industrial automation using robotic systems has been rapidly developed and perfected to perform several production tasks [1]. Spray painting, welding, assembly and material handling are some examples of the tasks that could be performed by industrial robots today.

The development of industrial robotic systems is a result of the progress in several disciplines such as mechanical, electrical, programming and control sciences. An industrial robot is a mechanically actuated mechanism that is controlled by a means of a computer system. Unfortunately, an exact definition of an industrial robot is not clearly provided as it is occasionally disputed amongst specialists [2]. In general, an industrial robot is a multipurpose mechanical system that can be programmed to execute various tasks. They are also characterized by operating in wide workspace compared to the volume they occupy. For example a computer numerical controlled (CNC) machine is not considered to be an industrial robot because it is specifically built for performing particular tasks. Their volumetric space is also much larger than their workspace field [1].

Industrial robotic manipulator is a mechanical structure that resembles the human arm. It consists of a series of links connected together with joints. It is fixed at the base on one end and at the other side it ends with an end-effector or tool. The movement of the robotic manipulator is being actuated at each axis of motion such as rotational and/or translational axis. The position 
and the velocity of each of the robot's joints are being determined by a means of internal sensors and encoders. The information determined by the sensors and encoders are fed back to the robot's controller which in turn calculates the predicted position and orientation of the endeffector. The controller then commands the actuators to implement the identified program under the guidance of a human control to drive the end-effector to reach the intended position and orientation. Due to several sources of inaccuracies, there is always a discrepancy between the actual end-effector's pose and the intended pose.

There are two main terms that are considered to be an attribute of the performance of robotic manipulators; accuracy and repeatability. The understanding of these two terms is mainly dependent on the understanding of the different error types. Errors can be divided into two main types; random errors and systematic errors. Random errors are due to unpredictable sources and they tend to vary the measurements uniformly around a true value. While the systematic errors sometimes called biases is the deviation of the mean value away from the true value. The repeatability of a robotic manipulator is the measurement of the precision or randomness of achieving the same pose of the robot's end-effector multiples of times under the exact same conditions. The accuracy of the robot's end-effector is the measurement of the closeness of the actual pose to the required pose, which is a direct representation of the system systematic errors [3]. In general, industrial robotic manipulators are characterized by their high repeatability which usually varies between the values of 0.1 to $0.03 \mathrm{~mm}$. On the other hand, the accuracy values of industrial manipulators are considered to be significantly worse than their repeatability values which usually lie in the order of $1 \mathrm{~mm}[4]$. 
Robotic manipulator's accuracy is an important factor in various industry applications. Welding, drilling and machining are some applications that require high accuracy systems to be performed. The position/pose of the end-effector with respect to the base of the robot or to an external frame of reference has to be accurate enough for performing these jobs. The inaccuracies encountered in any robotic manipulator could be divided into two main sources, geometric and non-geometric. First, geometric errors are a result of the robotic geometric parameters. Second, the non-geometric sources could be mapped to different factors such as gears backlash, encoders' accuracy, links flexibility and errors due to thermal and wear effects [5].

Any robotic manipulator could be mathematically represented as a non-linear function of the robot geometric parameters and joint variables. The robot geometric parameters are the parameters that represent the geometry of the links and their connection together. However, the joint variables are the representation of the joints displacements, for example, a revolute joint would be presented by an angle and a prismatic joint would be presented by a length. Hence, there are two main approaches investigated by researchers and used by industry to significantly increase the accuracy of robotic manipulators. The first approach is called calibration which is mainly concerned by adjusting the geometric parameters of the non-linear function to eliminate the end-effector errors. The second approach is called compensation which focuses on correcting the tip errors by changing the joint variables of the robot, in other words, controlling the displacements of joints of the robotic arm.

As a result of the inherent convenience of the calibration approach with respect to the compensation techniques, calibration became the main focus of researchers for further 
development during the last few decades. Many techniques and algorithms have been developed to improve the accuracy of robotic manipulators. Furthermore, many software packages were created by robotic manufacturers such as ABB CalibWare which provide an easy and quick tool for calibration.

Calibration process is divided into four main steps which are kinematic modeling, measurement, identification and finally implementation. Kinematic modeling is the very first step that is required for the calibration process. Kinematic modeling is basically formulating a mathematical model that represents the robotic manipulator as a function of its geometric parameters and joint variables. The second step is the measurement system which provides sufficient information of the robot's hand position and orientation corresponding to multiple configurations of the manipulator. The third step is called the identification step which is mainly concerned with using the kinematic model and the measurement information to estimate the parameters that reduces the tip errors. Finally, implementation or correction is the step that investigates the usage of the estimated robot's parameters to physically increase the robot's accuracy $[5,16]$.

The main objective of any calibration problem is finding the geometric parameters that drive the residual errors towards zero. Calibration is an iterative process that starts with an initial guess which is close to the actual value, where the initial guess is the robot's nominal geometric parameters based on the robot's CAD models. In an essence, any calibration process is considered to be multivariable nonlinear optimization problem [16]. There are many ways for solving an optimization problem depending on the nature of the problem. The solutions could be divided into two main categories, namely linear and non-linear. The first approach is done by 
approximating the whole system with a linear model and then solving it. The second approach deals with the system as a non-linear problem using multiple ways for finding good estimates. As a result of the advancements in computer and computational fields, search methods became a popular non-linear approach for many researchers. One of the main advantages of the linear approach is having a fast convergence rate towards parameters estimates. On the other hand, linear approach exhibits many difficulties in certain conditions such as matrix singularities. This could be avoided using non-linear approaches which are characterized by a relatively slow convergence rate.

Robots manufacturers usually provide their customers with software programs or services as an easy to use calibration packages. Since robotic arms have several applications, users customize the robot for serving their needs by designing different tools and gadgets which are mounted on the robot's end-effector. Due to manufacturing and assembly errors encountered in attaching an extra tool link to the robot, the overall accuracy of the robot reduces significantly. This forces users to perform a secondary calibration process to correct the errors of the tool link. The calibration of the tool link is done in an ad-hoc fashion, in other words, case by case scenario. It is also usually done for specific axes, which are most relevant to the application and not for the full pose. This is a tedious, time and money consuming process which also lacks any systematic approach and heavily depends on the skill level of the technician performing the calibration.

The main topic presented in the following thesis is focusing on developing a new systematic calibration method for correcting the geometric parameters errors of the robotic mounted-tools. The method in this thesis is developed based on the following assumptions: 
- The geometric parameters errors of the robotic manipulator have to be calibrated to a sufficient accuracy. In accordance to that condition the robotic manipulator was considered to be perfectly accurate with zero errors. The following assumption is important as the transformation vector from the robot's end-effector flange to the robot's base should be perfectly known.

- The intrinsic parameters of the 3D vision system as well as the camera system have to be determined and calibrated. The vision system and camera have to be calibrated so they could accurately capture tip errors.

The main objectives of this thesis are summarized and listed below in the following points:

- Develop a new robotic tool center point (TCP) calibration algorithm based on linear and nonlinear approaches. There are two linear approaches; the first approach is based on the conventional calibration technique using least squares while the second approach is based on the technique developed by Gong et al. [53] for calibrating a robotic manipulator system. The non-linear approach would be based on a widely used computer search algorithm called genetic algorithm (GA).

- Investigate both the linear and the nonlinear formulations and determine their limitations, advantages, disadvantages and effectiveness.

- Use a 3D vision system as well as the camera system for developing multiple techniques for the robot's end-effector position estimation and the tool orientation estimation. 
- Verify the developed techniques via computer simulations and determine the limitations and conditions for conducting experiments.

- Finally, conduct an experiment to validate the simulation results and the overall designed calibration formulation.

\subsection{Thesis Organization}

This thesis is divided into six main chapters in addition to the introduction. In this section the organization and a complete outline of the thesis is provided as well as a concise description of the contents of each section.

Chapter 2 is a complete survey that covers all of the topics introduced in this chapter. The chapter starts with the four main steps of the calibration process, which are modeling, measurement, identification and implementation. In addition, a complete section was fully dedicated to discuss the self-calibration methods and techniques. Finally, the last two sections provide an overview of the differences between the tool center point (TCP) calibration and the hand-eye calibration.

Chapter 3 discusses the very first step of building a calibration model which is creating a forward kinematic model for the given system. The problem of calibration is discussed and the basis of the kinematic model would be introduced. Then, a full system description is provided. Finally, an error model is created to describe the relation between the tip pose errors and the links geometric errors.

Chapter 4 presents the process of building a calibration formulation based on the system's kinematics and error models derived in chapter 3. The first calibration model is based 
on the commonly used approach of linearizing the forward kinematic model of the system using the conventional calibration as well as the self-calibration algorithms. Afterwards, a nonlinear formulation is developed using genetic algorithm as a search method for determining the geometric errors. A comparison between different measurement techniques for the different calibration formulations is discussed.

Chapter 5 starts with introducing the measurement system used for the calibration process developed in this thesis. Then multiple techniques of tool pose estimation and end-effector position estimation will be presented and simulated. The simulations in this chapter provide the limitations and conditions that must be taken into consideration for performing the experiments.

Chapter 6 provides the experimental set-up for the end-effector position estimation as well as for the tool pose estimation. The calibration algorithms are then applied and the results obtained are presented and discussed.

Chapter 7 presents the final conclusion of the thesis, the contributions as well as the proposed future work. 


\section{CHAPTER 2: LITERATURE REVIEW}

This chapter includes a general coverage of the different topics that were introduced in this thesis as well as the previous research that was conducted on each of these topics. The literature review will cover the following topics. The first topic is a brief description of the kinematic models that were developed specifically for the purpose of calibration procedure. The second section of this chapter is divided into multiple parts that introduce different measurement technologies and techniques for the calibration process. The third topic will summarize the methods developed for solving the calibration formulation using linear and non-linear approaches. Following this section is an explanation of the implementation methods used for achieving a complete calibration procedure. Afterwards, a whole section is dedicated for providing a quick glimpse of the importance of the self-calibration methods and some of the techniques used by different researchers. This Chapter ends with two sections that are mainly concerned by clarifying the differences between tooling calibration and hand-eye calibration techniques. There is also a full coverage of all the techniques developed for tooling calibration and the most famous hand-eye calibration methods. 


\subsection{Kinematic Modeling for Manipulator Calibration}

There are two main types of models for any robotic manipulator which are the forward model also known as Direct Geometric Model (DGM) and inverse model or Inverse Geometric Model (IGM). The first is the Direct Geometric Model (DGM), its main purpose is to mathematically relate the output of the joints movements to the final end-effector position and orientation. The second is the Inverse Geometric Model (IGM) which aims at using the knowledge of the end-effector position and orientation to find all the corresponding joints movements. Any calibration process can be divided into four main steps that start with kinematic modeling. Kinematic modeling for calibration is mainly concerned with the first type which is the Direct Geometric Model (DGM).

Any robotic manipulator is composed of a combination of links connected together by one of two types of joints. These joints are the revolute joint and the prismatic joint. Any other complex type of joint could be expressed as a compound of these two types of joints with no links in between [5]. Both joints are characterized by having one degree of freedom as the revolute joint provides a rotation around a fixed axis, while the prismatic joint provides a translation along a fixed axis.

There are multiple methods that could be found in literature regarding the development of kinematic models for calibration purposes. The effectiveness of each of these models can be described based on three main factors which are completeness, equivalence and proportionality [6]. First is completeness of the model which denotes the availability of enough variable parameters that fully describe the motion of the manipulator. Second is the model equivalence which means the ability of conversion from one complete model to another. The Third and last 
factor is the model proportionality which means that any variation in the robot should be proportionally represented by a variation in the model. The lack of model proportionality with the actual model may lead to a numerical instability during the calibration process.

The robotic system/workspace is composed of multiple objects moving in space in relation to each other. The basic problem is the ability of expressing the position and orientation of all the workspace objects and their relation to each other. Kinematics is a field of classical mechanics that is concerned with describing the relative position, orientation and motion of different bodies with each other. A robotic manipulator can be described as a series of links connected with joints and can be expressed mathematically by attaching frames to each link. The relation between the frames can be described using several techniques and approaches. The most famous approach is the Denavit-Hartenberg parameters representation [7].

Using the four DH parameters that express the relation between the two links, a homogeneous transformation matrix can be constructed to transform from one frame to the other. An expression of the robot manipulator can be attained by combining all the homogeneous transformations from the base all the way to the robot's end-effector. The final transformation form is the relation between the robot's end-effector as a function of all the robot's joints which is known as the Direct Geometric Model (DGM). Many researchers have used the DH parameters method for kinematic modeling for their calibration problems such as Perriera [9], $\mathrm{Wu}[10,11]$, Zhen [12] and Payannet et al. [13].

Although being a successful method at the beginning, the Denavit-Hartenberg parameters approach was discovered later to have some major deficiencies especially for the calibration purposes. The major drawback of the $\mathrm{DH}$ parameters representation was discovered and 
discussed by Mooring [14] and Hayati [15]. It was discovered that there is a major discrepancy experienced by the applying the method in the special case of a robot designed with two or several parallel revolute joints. The general case, where there is a unique common normal between the two revolute joints. The uniqueness of the common normal does not exist when the revolute joints are parallel and it could be chosen anywhere. As a result of the calibration process, if the revolute joints axes were discovered to be slightly off their parallelism, at that instance the axes must have a unique common normal which might be in a totally different position from the assumption made at the beginning. Other problems such as the lack of the base frame location choice and the manipulator "zero position" were also considered as limitations of the DH parameters method [16].

The limitations and weaknesses of the Denavit-Hartenberg model was determined and carefully studied by many researchers. Many researchers have introduced some modifications to the DH parameters representations to avoid the undesirable discontinuity due to parallel revolute axes. Hayati et al. [17] have proposed a method that replaces the common normal with a plane perpendicular to the first joint axis and includes the origin of its frame. The point where the plane intersects with the second joint axis is the origin of the second joint frame. The direction of the $\mathrm{x}$-axis of the second frame is determined by the direction of the line connecting the origins of the two frames. Other methods were also developed to solve the DH parameters method such as Ibarra and Perreira [9] by using an error matrix and Hayati [15] by introducing a method to describe the error of the axes that are not parallel. The modified $\mathrm{DH}$ model was used by a number of author in different applications is such as Gatla et al. [24] and Ligtca et al. [25]. 
Another model was formulated by Stone et al. [18] is called the S Model. A frame is attached at each joint and then related to each other using 6 parameters as a modified version of the DH parameters model. This model is considered to be incomplete since the base frame could not be arbitrarily located [19]. More complex models were developed later with more sets of parameters to provide a complete description of the manipulator. As a consequence parameters identifiablility methods were developed for reducing the number of parameters to avoid the Jacobian matrix singularity [16].

A method that does not demand the need of the manipulator's geometry and does not provide inconsistent results in special cases such as the DH parameters model was discussed by Mooring [14]. The method uses six parameters to represent the relation between each of the frames attached to each link. The six-parameter model uses three parameters as a vector to locate the position of one frame with respect to another one. It also uses three more parameters to express the orientation of the frames with respect to each other. There are different methods of describing the orientation of frames such as the Roll, Pitch, Yaw method or Euler angles method [2]. The six-parameter model was used for robot calibration modeling by several researchers such as Whitney et al. [21], Hollerbach [22] and Chen et al. [23].

\subsection{Measurement Tools}

The robotic manipulator is a system that could be described by an input-output relationship. The robot's end-effector pose is the output of the combination of several links/joints geometries and displacements inputs [26]. The kinematic model of the robot manipulator covered in the previous section relates the end-effector pose output to the joints inputs. By determining the actual output of the system through measurements and comparing it to the 
expected output, the errors could be mapped to the corresponding inputs. Mapping techniques to find the actual inputs is the topic of the third step of the calibration process covered in the following section.

As mentioned in the introduction of this thesis that the calibration is a process that is divided into four main steps in which measurement comes as the second step. The measurement step for the calibration process is mainly concerned by determining the full pose or a partial pose data of the end-effector given a combination of the joints configurations. Usually multiple measurements are needed for performing any calibration process for two main reasons. The first reason is to eliminate the measurement noise or the random errors. The second reason is to find the best set of parameters that minimizes the errors in, preferably, all the possible configurations within the workspace. The measurement process is usually done by exciting the robot's joints to reach a certain configuration. The joints' displacements are recorded and the robot's end-effector pose is measured. The process is repeated for multiple robot configurations to gather enough measurement data $[16,27]$.

The two main elements that affect the measurement process are the chosen measurement system itself and the measurement technique. There are different measurement-systems that will be introduced and discussed in brief in this section, in depth knowledge of these systems are found in [28]. The measurement system should satisfy certain criteria regarding the calibration process such as accuracy, repeatability, effectiveness and easiness. In this section the six most famous measurement systems would be introduced, these systems are the laser trackers, laser interferometers, Coordinate Measuring Machines (CMMs), cameras, theodolites and wire-draw 
encoders. There are also other systems such as inclinometers, ball-bars, short range and time of flight devices that will not be discussed.

\subsubsection{Laser Tracking Systems}

The laser tracking system is considered to be one of the most advanced measurement tools as it has a high accuracy of $0.1 \mathrm{~mm}$ or even less over a range of few meters. Shown in Figure 2-1 below, is a diagram of the basic concept of the laser tracking system operation. The system is composed of two receiver units $\mathrm{T} 1$ and $\mathrm{T} 2$ and a retroreflector target. The receiver shoots a laser beam at the retroreflector target mounted on the robot's end-effector. The beam gets reflected back to the receiver which uses the polar and azimuth angles to calculate the position of the end-effector. There are more advanced laser tracker systems that use absolute distance measurements in addition to the two angles such as utilized by Yurtagul [26], Gong et al. [29] and Meng et al. [31].

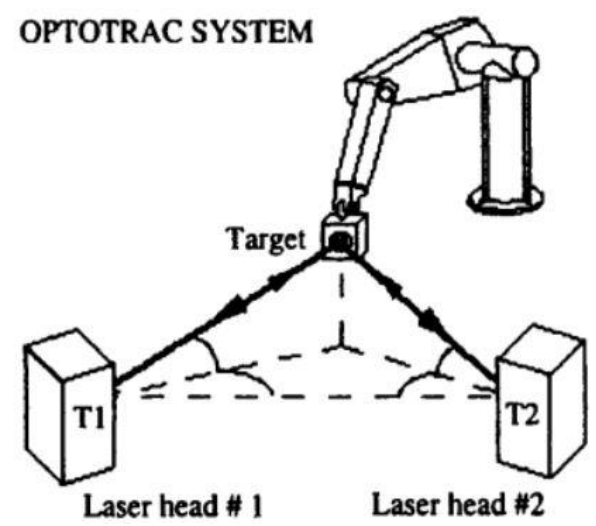

Figure 2-1: Laser Tracking Concept Diagram [5].

\subsubsection{Laser Interferometers}

Laser interferometers are devices that measure the linear distance of an object with respect to its position. The basic principle of the laser interferometer operation is based on light 
interference phenomena. The basic structure of a laser interferometer is shown below in Figure 2-2. It is composed of a laser device that emits a laser beam through a beam splitter which splits it into two beams and then recombines them on the surface of a photodetector. The interference between the original beam and the reflected one creates a pattern which enables the measurement of the distance. In some modern devices the laser interferometer is combined with the laser tracker in one unit that measures the distance and the angles and determines the position of the target.

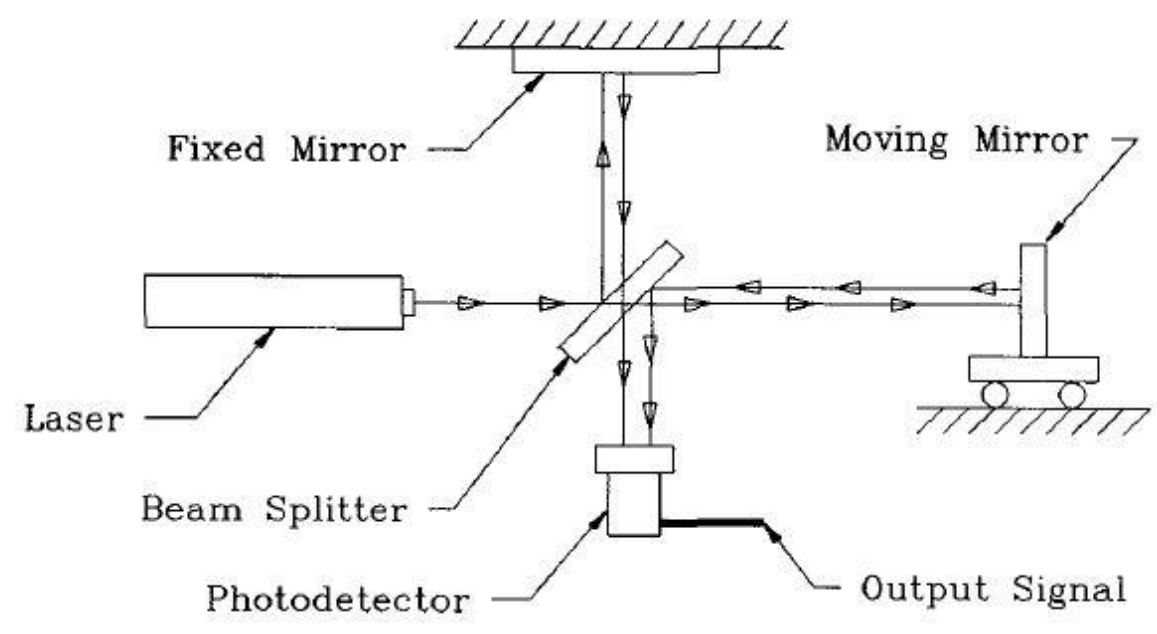

Figure 2-2: Laser Interferometer Concept Diagram [16]

A laser interferometer and an optical sensor were used by Majarena et al. [30] to calibrate a parallel mechanism. The laser interferometer used is considered to be a very accurate linear displacement device with an accuracy of $0.02 \mu \mathrm{m}$. It was used for calibrating non-geometric errors such as gear backlash of an order $53 \mu \mathrm{m}$.

\subsubsection{Coordinate Measuring Machines (CMMs)}

Coordinate measuring machines (CMMs) are very precise and accurate mechanical machines that could determine the location of a point in the space. CMMs could reach very low 
accuracies on the order of $0.01 \mathrm{~mm}$. Usually CMMS are large expensive mechanical machines, shown in Figure 2-3, which are not considered to be a convenient measurement device in an industrial environment. Some portable CMMs were developed for higher convenience but with much lower accuracy. CMMs are usually used in lab experiment set-ups rather than being used for industrial purposes.

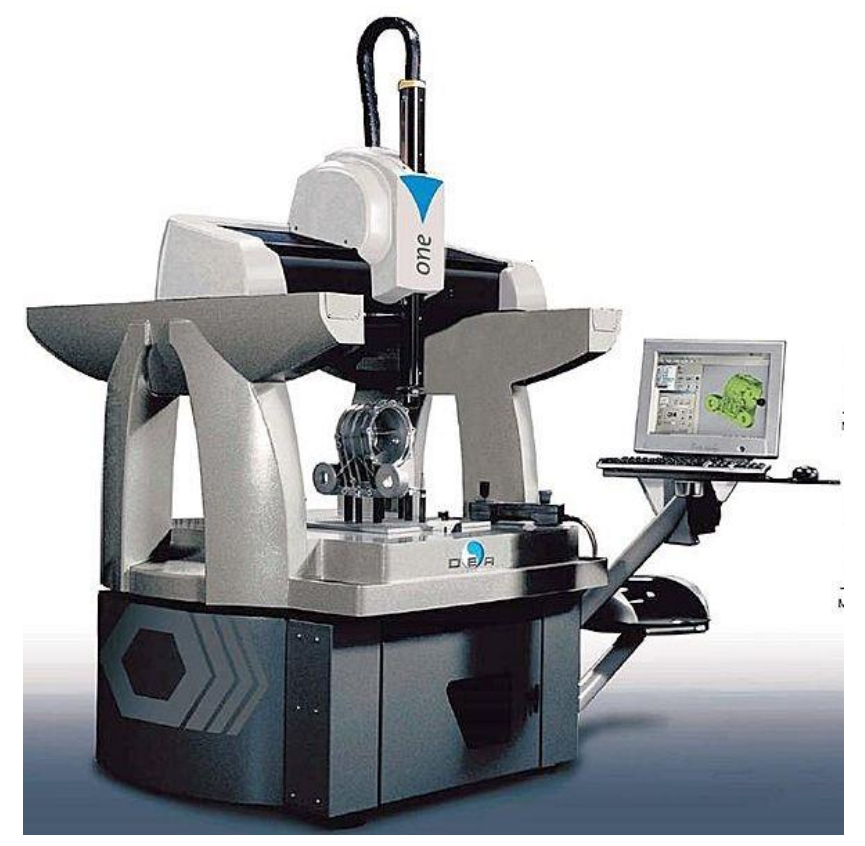

Figure 2-3: Modern CMM Machine [32]

The positioning accuracy of PA10-6CE was significantly reduced from $1.8 / 2.45 \mathrm{~mm}$ to 0.33/0.71 $\mathrm{mm}$ by Lightcap et al. [25]. An aluminum frame was attached at the Robot's endeffector which ends with three spheres. A CMM machine was used to take multiple measurements of the surface of each sphere and the least square method was used to determine the center of the spheres.

Mooring and Padavala [33] used a small CMM machine with a repeatability of $0.1 \mathrm{~mm}$ and accuracy of $0.1 \mathrm{~mm}$ to develop a pose measuring system. The system was used to collect 
data for 90 poses to determine the parameters of five different models for their PUMA 560 robotic manipulator.

\subsubsection{Camera Type Devices}

Another device of a great interest to researchers for robotic manipulator calibration is the charged couple device (CCD) camera. The camera system is composed of a lens and a 2D light sensitive array that is a structure of number of pixels. The more the number of pixels in the array, the better the resolution of the camera is and hence a better accuracy. A camera with $512 \times 480$ pixels resolution was used by Zuhuang et al. [34] for developing a kinematic calibration model for PUMA robotic arm.

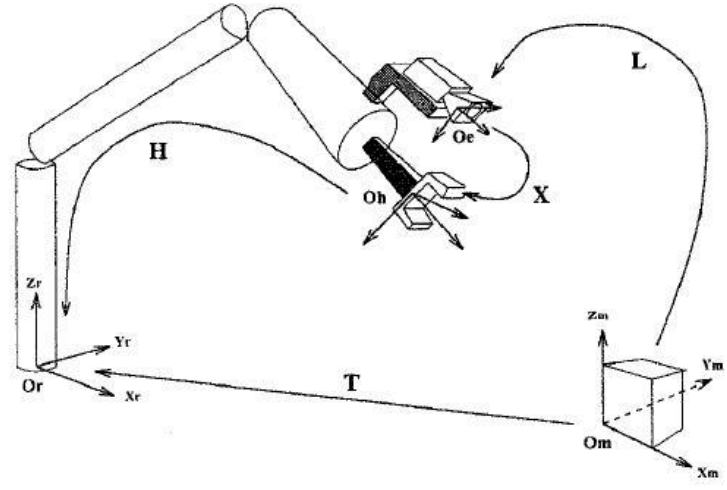

Figure 2-4: Moving Camera Setup [36]

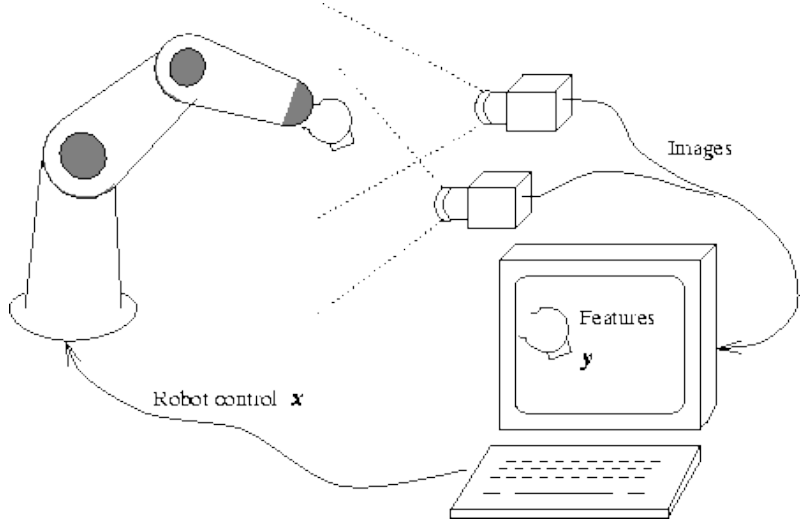

Figure 2-5: Fixed Camera Setup [38]

There are two main camera setups used by researchers which are the moving camera setup and the fixed camera setup. The first setup, shown in Figure 2-4, is the moving camera setup which is done by mounting a single or a stereo camera system on a robotic arm endeffector. The second setup, shown in Figure 2-5, is the fixed camera setup which is done by fixing a camera on the workspace of the robot. The second approach has a major disadvantage which is the need of a high resolution camera with a large field of view. These two conditions are usually hard to combine since one is complimentary to the other. On the other hand the first 
approach is considered to be more efficient than the second approach since the camera system has to only detect the calibration pattern rather than the whole workspace.

A camera feedback system was used by Gatla and Lumia [24] to detect the laser point controlled by a robot on an arbitrary plane. The system was also used to change the robot configurations to move the laser point to the required position. The resolution of the camera system used was $0.05 \mathrm{~mm}$ which corresponds to 1 pixel.

A CCD camera system mounted on the robot's end-effector was used by Motta et al. [37] to develop a calibration algorithm based on 3D vision based measurement system. The method developed was proved to achieve an accuracy that ranges from $0.2 \mathrm{~mm}$ to $0.4 \mathrm{~mm}$. The calibration process was experimentally applied on two robotic systems which are the ABB IRB2400 and the PUMA-500 robots.

\subsubsection{Theodolites}

Theodolite, shown in Figure 2-6, is basically a small telescope which is mounted on two rotary axes as the laser tracker receiver. The line of sight could be determined accurately using two angles which are the polar and azimuth angles. Using two units such as shown in the diagram in Figure 2-7, the position of a certain target could be accurately determined. The accuracy range of the theodolite is very good and has the order of $0.02 \mathrm{~mm}$. Old versions of the

theodolites were operated manually and the data were calculated and stored by the means of a computer. The modern theodolites were designed to be fully automated to track a lighting object mounted on the target intended to be measured. A detailed description and explanation of modern theodolites could be found in Cooper [35]. 


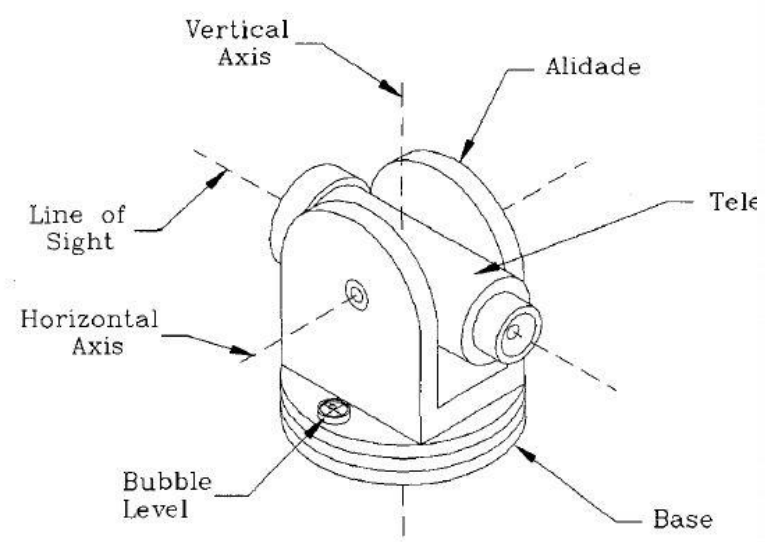

Figure 2-6: Theodolite [16]

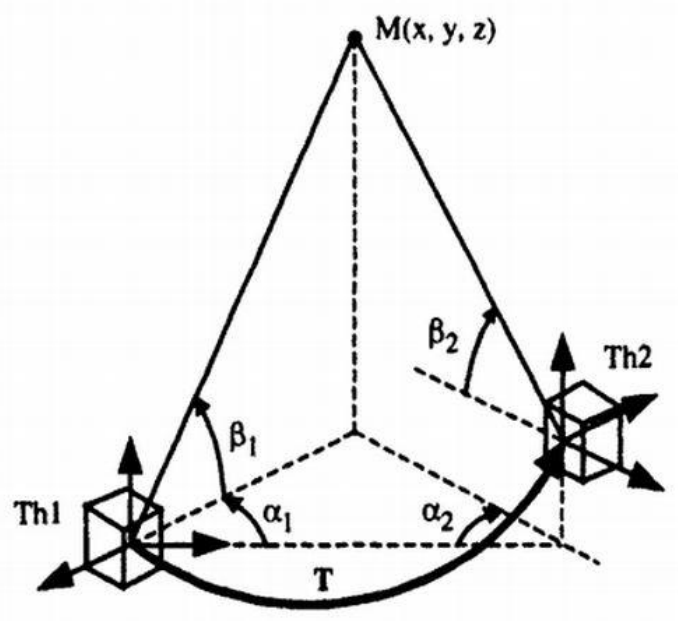

Figure 2-7: Theodolite System Diagram [5]

Chen and Chao [23] have used three theodolites with stereo-triangular technique for their measurements. The three theodolites were operated manually and the data were collected and calculated by a computer. The accuracy of their measurements was fully verified to be in the order of $0.02 \mathrm{~mm}$ within $1 \mathrm{~m}$. these measurements were used for developing a calibration algorithm for reducing the position errors of the PUMA 760 robotic arm from $5.9 \mathrm{~mm}$ to 0.28 $\mathrm{mm}$.

Whitney et al. [21] have also used theodolites measurements to develop a calibration algorithm for serial link manipulators. They have used theodolites to measure the tool position in addition to the robot's joint encoders. The calibrated technique used was verified to reduce the manipulator's error for as low as $0.3 \mathrm{~mm}$.

\subsubsection{Wire-Draw Encoders}

A robot tracking system called (ROBOTRAK) used by McMaster et al. [71] for robot calibration and tracking. ROBOTRAK, shown in Figure 2-8 below, is a system that uses three 
wire-draw encoders which are connected to the robot tip via three wires. The system operates based on triangulation method. The encoders are located in known locations in space and continuously measure the length of the three wires. The robot tip position could be easily calculated using the relative positions of the encoders in addition to the wires lengths.

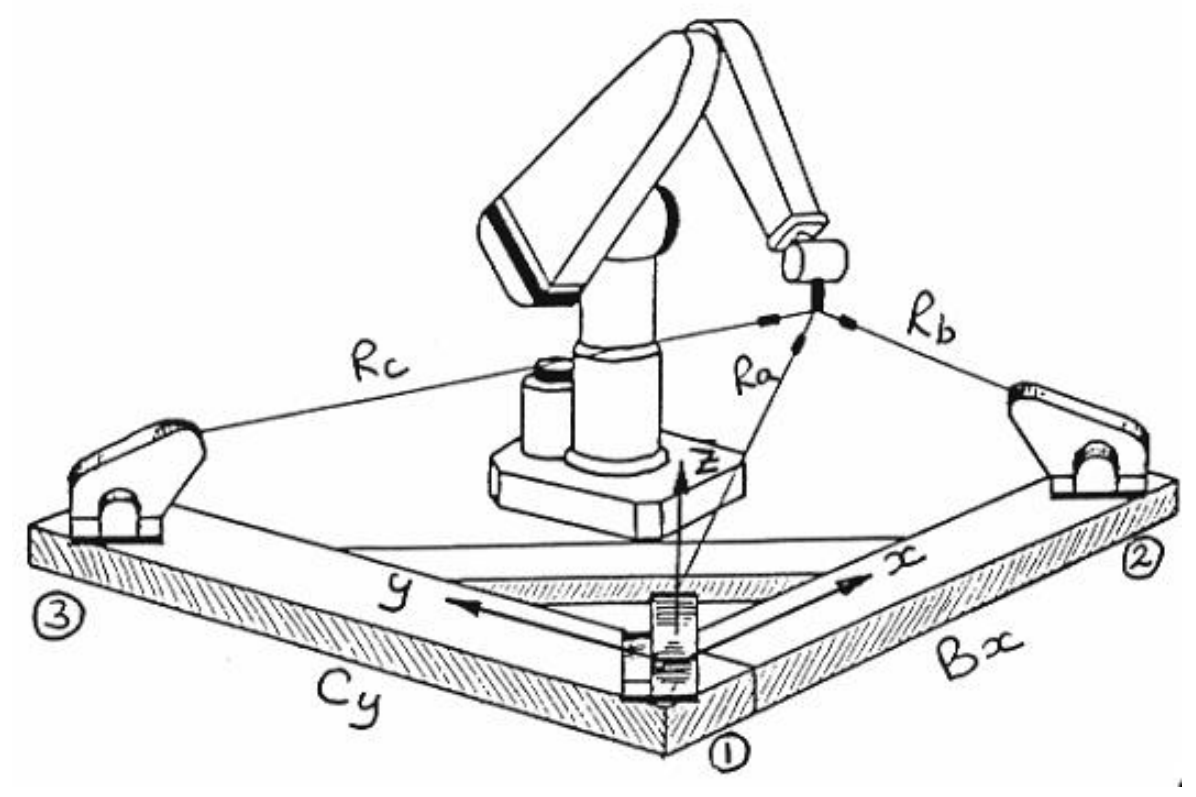

Figure 2-8: ROBOTRAK Measurement System [71]

The absolute accuracy of the robot track system was determined to be $0.5 \mathrm{~mm}$ in a range of $2 \mathrm{~m} \times 2 \mathrm{~m} \times 0.7 \mathrm{~m}$. The system is also capable of performing robot tip path measurements, dynamic analysis and recording the tip velocity and acceleration.

\subsection{Identification}

Identification of the robot's kinematic model parameters is the third step of the calibration process after modeling the robotic manipulator and gathering a set of measurement data. The main objective of the identification process is finding a set of geometric parameters 
that minimizes the errors between the model's predicted values and the measured data. The identification step has three basic requirements; first, a mathematical model of the robotic manipulator. Second, undetermined variables that requires estimation which are usually the unknown parameters given in the mathematical model. Third requirement is a set of measurements that relates the output to the input of the mathematical model [16].

As mentioned in the thesis introduction, the calibration problem is mathematically considered to be a multivariable nonlinear optimization problem. Identification methods could be divided into three main categories. First, linear approach and nonlinear approach where linear approach is mainly determined by linearizing the robot's mathematical model or leave it as a nonlinear problem. Second, recursive method or non-recursive method where the first requires a set of parameters that has to be updated through an iterative process and the second is just a direct estimation of the set of parameters. Third, deterministic and stochastic techniques which depend on the treatment approach of the measurement noises in the developed model. [16]

The two main approaches for parameter identification are the linear and the nonlinear approaches which have their own advantages and disadvantages. As observed by Pathre et al. [39] that the linear approach leads to convergence of the errors approximately ten times faster than that of the nonlinear approach. Even though the linear approach leads to a faster convergence, the nonlinear approach is considered to be more robust than the linear approach in determining large errors. Linearizing the robot's kinematic model is essentially done by using Taylor expansion and ignoring the higher order terms which leads to the determination of the Jacobian matrix. This is basically what is known as the error model which could be solved using linear least-square methods. Using a linearized model in addition to an ordinary least squares 
technique in an iterative approach is known as Gauss-Newton algorithm. Gauss-Newton algorithm leads to a fast convergence under two main conditions; starting with good initial values such as the robot's nominal geometric parameters and the model should not exhibit any severe nonlinearity [40].

A modified algorithm of the Gauss-Newton technique was introduced by Levenberg [41] and Marquardt [42] and was named after them as the Levenberg-Marquardt algorithm. This algorithm was developed to solve Gauss-Newton technique limitations as it does not provide parameters estimation in case of singular matrices. It was noted that Levenberg-Marquardt algorithm performs well in all conditions even for large errors but with a slow convergence rate [16]. This algorithm was used by Chao and Yang [43] to prove the validity of the method by applying it on the data presented by Chen and Chao [23] and yielding the same results.

Researchers have used different techniques for the parameters identification problem to improve the efficiency of the calibration processes. A linear Cartesian error model was used by $\mathrm{Wu}[10]$ to generate Cartesian error envelopes which could be minimized. Another method was proposed by Yahui and Xianzhong [45] which basically depends on representing the rotation matrices by quaternions and then using Levenberg-Marquardt method to solve the nonlinear equation. Wand et al. [46] presented a method that uses screw axis identification (SAI) method based on the product of exponentials (POE) model. It was used for calibrating a robotic manipulator with a stereo-camera vision system. A two-level nonlinear optimization using Levenberg-Marqardt approach was used by Lightcap et al. [25] to improve the positioning accuracy of the PA10-6CE Robot. 
There are other popular nonlinear approaches that have been widely used as a result of the advancements in the computation techniques and computers. Search algorithms are considered to be a reliable and effective type of techniques. Genetic algorithm is a search algorithm that was used by multiple researchers for calibration purposes. Lin et al. [47] have used genetic algorithm combined with Monte Carlo simulation for the calibration of modular reconfigurable robots (MRRs). Even though genetic algorithm is a reliable technique and usually leads to convergence, one major disadvantage of search methods in general is their relatively slow rate of convergence.

The main focus that is of a great concern at this stage of the calibration process is the set of data measurements acquired. The identification process of the parameters could be greatly affected by the measurement noises in addition to the unmodeled errors. As a result, the reduction of measurement noises is an important aspect of the parameters identification process. The main defined problem is determining the minimum set of robot configurations during the measurement process that reduces the measurement noises. The optimal set of robot configurations could be easily done by using simulation experiments to perform an observability analysis as presented in Joubair et al. [27], Zhuang et al. [48] and Sun and Hollerbach [49]. The observability analysis is usually performed using one of two methods which are singular value decomposition (SVD) or QR decomposition of the Jacobian matrix. The SVD could be used to maximize the values of what is known by the observability indices. Joubair et al. [27] used the SVD approach to compare between two calibration algorithms for six-axis industrial robot. Another approach is the analysis of the condition number of the Jacobian matrix which was proved to be related to the observability indices by Driels and Pathre [50]. The condition number analysis was used by Watanabe et al. [51] to determine the set of required measurements and it 
was concluded that the condition number is a good indication of the stability and effectiveness of the identification process. Khalil et al. [52] used the QR decomposition technique for determining the parameters identifiability and concluded that a random set of robot configurations provide a good condition number.

\subsection{Implementation}

Implementation or sometimes called "verification and correction" is considered to be the last and final step of any calibration process. This step is mainly concerned with using the calculated information of the robot's parameters to actually improve the accuracy of the robot manipulator. There are two basic approaches for correcting the manipulator's parameters. First method is the correction of the robotic manipulator's parameters inside the controller. Unfortunately, there are two main problems that are of great importance which are the modifiability of the controller's software and the type of the model saved in the controller. There are some controllers that do not allow the adjustment of the model's nominal parameters. There are also some controllers that include only a geometric model type of the manipulator and do not include the non-geometric parameters. So as a solution of the modifiability problem, the second method is to adjust the targets positions in the controller instead of adjusting the robot's parameters. Adjusting the positions of the targets is called creating fake targets. [26]

There are two important robotic applications which are the off-line programming application and the teaching application. The off-line programming application is a modern method that uses a simulation of the robotic manipulator from a CAD or CAM program to control the actual robot on the workshop floor. The teaching application is the method of directly

programming the robot's joints to do perform a certain moves. Unlike off-line programming 
application, teaching application does not require the knowledge of the robot's kinematic model. For the off-line programming application the inverse kinematic model is an essential requirement for the calculation of the joint's angles. Therefore, the implementation step is absolutely necessary for an accurate inverse model. Implementation is also necessary for teaching application in case of changing robots which requires an algorithm that adjusts the joints angles at each instant [16].

\subsection{Self-Calibration}

As mentioned in the previous sections of the literature review, there are many methods and techniques that were developed for performing a complete robotic manipulator calibration. Various measurement techniques were presented in the measurement section such as Coordinate Measuring Machines (CMMs), Laser trackers, theodolites and Cameras. Unfortunately, most of the calibration techniques developed and the measurement systems used are difficult and expensive to be applied on the workshop floor. As a result, researchers have been investigating a type of manipulator calibration that is easy to be implemented on the manufacturing floor known as self-calibration. The main advantage of the self-calibration techniques is that they do not require external measurement devices and systems.

A self-calibration algorithm was developed by Gong at al. [53] for the calibration of serial manipulators. The method requires a light sensor to be mounted at the robot's end-effector which has the ability to find the center a hole. Using a calibration jig with multiple holes, the sensor is guided using the robot to find the center of each of the holes. Then using an algorithm that was derived based on the relation of the holes with respect to the robot configuration, the robot parameters could be identified through an iterative process. Another method was 
developed by Gatla et al. [24] using only the robot joint sensors and encoders and since there are no external measurement systems used for this algorithm, it is considered to be a self-calibration method. In this method a laser pointer mounted at the robot's end-effector which points to a constant arbitrary location. Then using the joint encoders the robot parameters could be

effectively determined. There are different types of the self-calibration method which mainly depend on the constraints. The first method is using location or position constraint. A different type is using a distance measurement such as used by Gatla et al. [24]. Another type is using a plane constraint such as the method used by Zhuang et al. [54].

\subsection{Tool Center Point (TCP) Localization}

As clearly mentioned in the introduction, the main objective presented in this thesis is developing a systematic calibration algorithm targeting the robot tool center point. A lot of attention was directed towards the calibration of the robotic manipulator itself without any concern of the application. As a result, many calibration techniques have been developed to increase the accuracy of robotic manipulators. In other words, the calibration techniques developed mainly focused on increasing the accuracy of the robot's end-effector position with respect to the robot's base frame. There are also many calibration software programs that were developed for facilitating the calibration of these robotic manipulators such as ABB CalibWare. The main problem manifests itself as the manufacturers of such manipulators ship them to the users. Each user uses the robotic manipulator for a different application which requires a different tool to be mounted on the robot's end-effector. Although the robot could be fully calibrated by the manufacturer or by any calibration software, the user experiences significant tip inaccuracies. Manufacturing tolerances and assembly errors of the tool mounted by the user 
affect the overall accuracy of the system. This problem leads the users to perform a secondary calibration to correct the errors created by the mounted tool. Users tend to perform this calibration in an ad-hoc fashion or on an axis by axis basis. This approach is a time and money consuming method as well as it depends on the skill of the workers performing the calibration. It is also considered to be an ineffective method in certain cases such as in the case of a tool exchanger mechanism or in applications that require the calibration of all the axes.
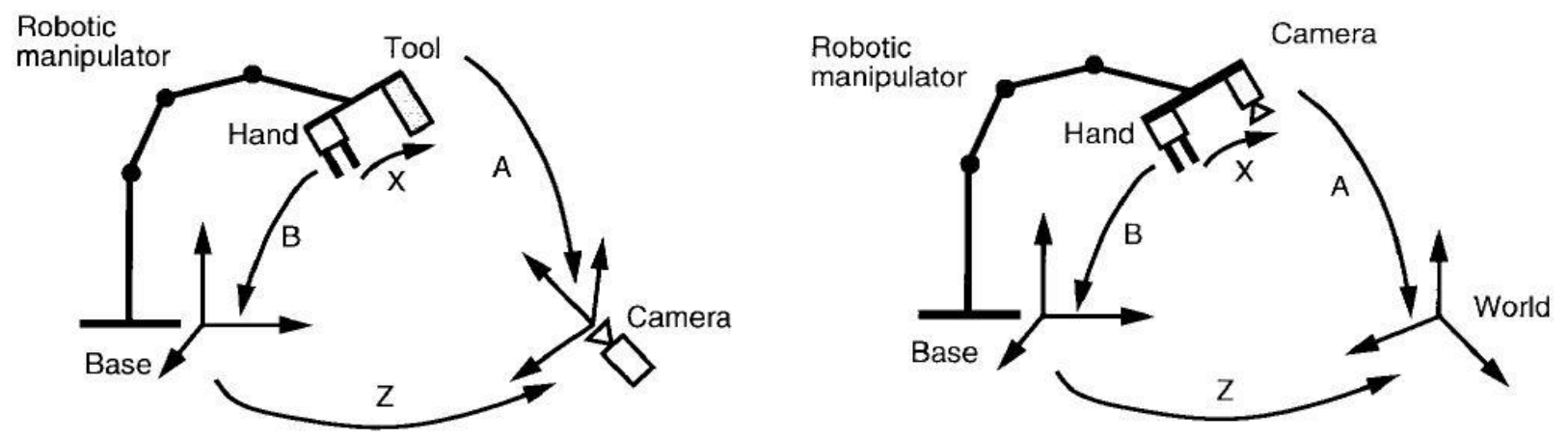

Figure 2-9: Hand-Tool vs. Hand-Eye Diagram [55]

The calibration of the robotic tool center point using a camera system is sometimes referred to as hand-eye calibration. There are many papers found regarding hand-eye calibration techniques such as (partial list) [56], [57] and [58]. Only few were found to clearly mention the distinction between the robot's hand (end-effector) and the mounted tool such as mentioned by Dornaika and Horaud [55] and shown in Figure 2-9.

The latest techniques used for robotic tool center point calibration could be divided into two main approaches. The first approach was presented by Hallenberg [59] for industrial usage and by Ayadi et al. [60] for medical applications. The second approach was developed by Cheng [61] for industrial application. 


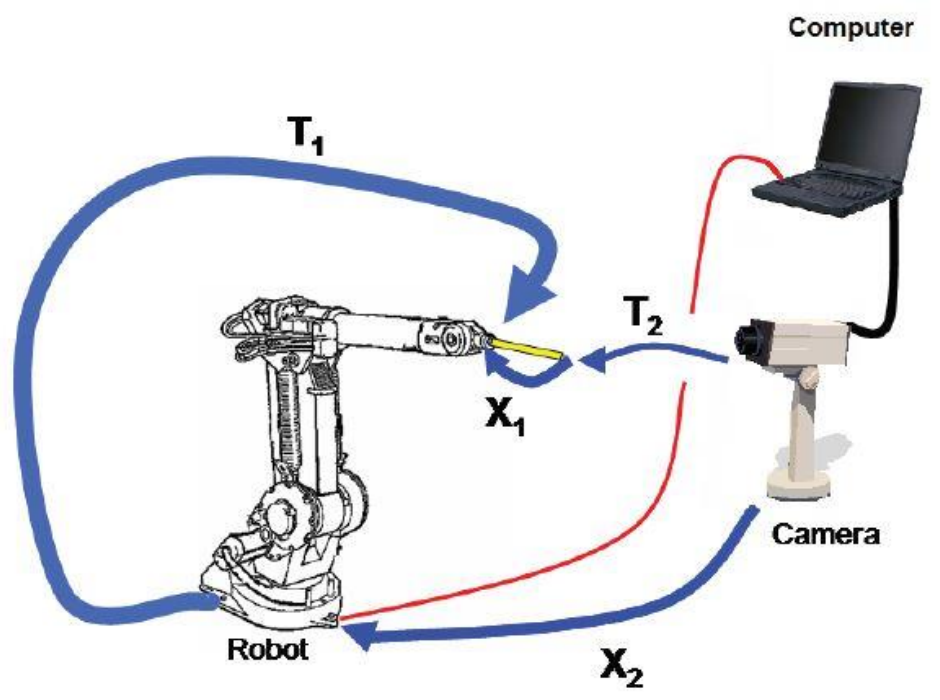

Figure 2-10: Tool Center Point Calibration Setup [59]

Hallenberg [59] proposed a system that is composed of a robotic manipulator with an industrial tool mounted at its end-effector. A single camera is used for the calibration procedure in addition to a computer with the developed algorithm. The robotic manipulator was assumed to be calibrated to a reasonable accuracy, so that the transformation from the robot's base to the end-effector is accurately known. The main idea starts by calibrating the camera's intrinsic parameters and then taking an image of the tool mounted on the robot's end-effector. Using a few image processing techniques and algorithms on the image captured, the tool tip position could be easily extracted from the image. The next step is formulating the problem as $T_{2} X_{1}=X_{2} T_{1}$ as shown in Figure 2-10, where $T_{1}$ is the base - end-effector transformation, $T_{2}$ is the camera tool tip transformation, $\mathrm{X}_{1}$ is the tool tip - end-effector transformation, $\mathrm{X}_{2}$ is the camera - base transformation. The equation could then be solved for $\mathrm{X}_{1}$ which is the tool-hand transformation.

Ayadi et al. [60] focused on applying the same technique for robotic-assisted puncture on small animals. The technique was used to find the relative position of a needle mounted on a robot with respect to the robot's end-effector. A stereoscopic camera system was used to 
determine the needle tip pose instead of a single camera system. The technique used in this research was proven to reduce the tool errors to less than $0.35 \mathrm{~mm}$.

Cheng [61] proposed a totally different approach for the calibration of robotic tool tip. He introduced an external measurement system that uses a cable device that locates the tool tip with respect to a specific frame. Using multiple robot configurations as shown in Figure 2-11 below and specifically developed software, the robot's TCP errors could be significantly reduced.

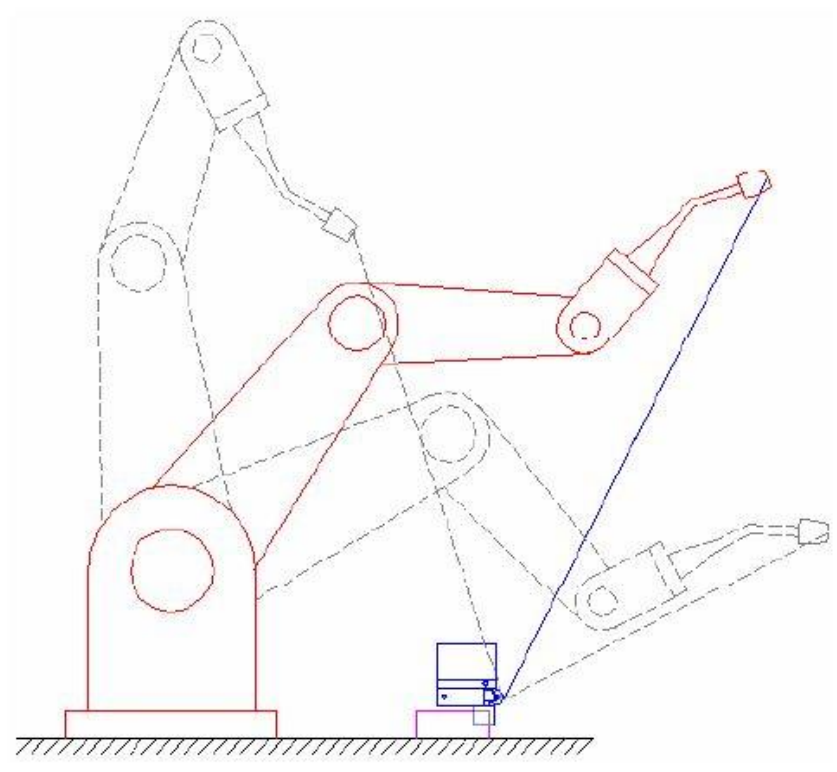

Figure 2-11: Multiple Robot Configurations [61]

\subsection{Hand-Eye Calibration}

Hand-eye calibration is a well-known problem that was completely identified and considered by researchers since 1989 . Hand-eye calibration is mainly concerned by a camera system which is mounted on the robot's end-effector and correcting the errors between the camera frame and the robot's end-effector frame. Due to assembly and manufacturing errors the position and orientation of the camera frame cannot be accurately determined with respect to the robot's hand (end-effector) frame. 
The calibration nature of this problem is slightly different from the conventional robotic manipulator calibration. The main difference lies in the fact that the transformation between the camera frame and the robot's hand frame is fixed and does not change. In contrast, a traditional robotic manipulator calibration problem has different transformations from the robot's base to the robot's hand which corresponds to different robot configurations. As a result, it is clearly noticed from the previous researches that researchers do not approach the hand-eye calibration using the traditional calibration approach. The traditional calibration approach is divided into four main steps which were explained in details in the previous sections, these steps are modeling, measurement, identification and implementation. The Hand-eye calibration is usually determined as a one-step accurate transformation and not as determining the errors for each single parameter.

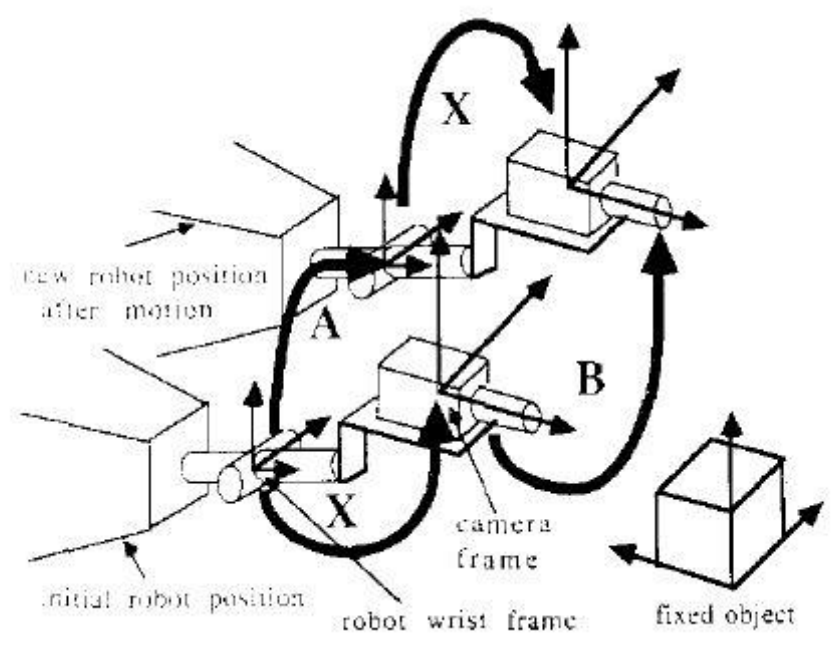

Figure 2-12: Hand-Eye Calibration Diagram [62]

A really well-known formulation that was proposed by Shiu and Ahmad [62] and developed later by many researches such as Park and Bryan [63], Zhuang and Shiu [64] and Daniilidis [65] is in the form of $\mathrm{AX}=\mathrm{XB}$ shown in Figure 2-12., where $\mathrm{A}$ is the transformation between the robot's end-effector (hand) in two different configurations, B is the transformation 
between the sensor's frames (Camera frame) in two different configurations and $\mathrm{X}$ is the transformation between the robot's hand and the camera's frame.

In this chapter the basics of the robotic manipulator's calibration process were introduced. Multiple measurement techniques and tools for conducting the calibration process were covered. Finally, different error identification techniques were discussed in addition to multiple calibration system set-ups. 


\section{CHAPTER 3: KINEMATIC AND ERROR MODELLING}

The very first step of building a calibration model for our system is by laying the basis of creating a forward kinematic model for the system. There are several approaches for building a kinematic model for a robotic arm manipulator discussed in different number of papers and texts such as [11,12] and [15-18]. One of the main approaches that are widely used is the four Denavit-Hartenberg parameters. Another approach is the six-parameter model that describes any robotic link in terms of three position vectors and three rotation angles. Since the DH parameters model was proven to exhibit several problems such as the violation of the proportionality requirement; the six-parameter model will be used to express the kinematic model in this thesis.

Creating a forward kinematic model for a robotic arm manipulator is essentially describing the position and orientation of the end-effector link as a function of the robot's geometric parameters and joints variable sets as described in Equation 3.1 below,

$$
\boldsymbol{X}=\left[\begin{array}{ll}
\boldsymbol{P}^{T} & \boldsymbol{\theta}^{T}
\end{array}\right]^{T}=f(\boldsymbol{q}, \boldsymbol{g})
$$

where $\boldsymbol{X}$ is the vector that describes the pose of the end-effect with respect to the base frame. $\boldsymbol{X}$ is composed of two vectors which are $\boldsymbol{P}$ and $\boldsymbol{\theta}$, where $\boldsymbol{P}=\left[P_{X} P_{Y} P_{Z}\right]^{\boldsymbol{T}}$ is the position vector from the base frame to the end-effector frame and $\boldsymbol{\theta}=\left[\theta_{X} \theta_{Y} \theta_{Z}\right]^{\boldsymbol{T}}$ is the vector of angles defining the orientation of the end-effector with respect to the base frame. $\boldsymbol{q}$ is a vector which expresses the joints variables set such as rotational angles for revolute joints and translational displacements for prismatic joints, while $\boldsymbol{g}$ is a vector of the geometric parameters set of the links.

The main problem is that the forward kinematic model does not describe the actual pose of the end-effector accurately due to several error factors such as manufacturing errors, assembly 
errors and other sources of errors. This means that the nominal kinematic parameters model predicts that the pose of the end-effector is given by $\boldsymbol{X}_{\boldsymbol{o}}$ while due to error sources, the pose of the actual end-effector is given by $\boldsymbol{X}_{\boldsymbol{m}}$. Then the difference $\Delta \boldsymbol{X}$ between the nominal pose and the actual measured pose shown in Equation 3.2 below is the kinematic parameters errors reflected at the tip pose.

$$
\Delta X=X_{m}-X_{o}
$$

There are two main methods for correcting the forward kinematic model to provide us with a better tip pose approximation. The first method is called compensation which is using the control aspect of the robot to correct the errors; that is correcting the tip pose errors by adjusting the joints variables as shown in Equation 3.3 below.

$$
\boldsymbol{X}=f\left(\boldsymbol{q}_{\boldsymbol{o}}+\Delta \boldsymbol{q}, \boldsymbol{g}_{\boldsymbol{o}}\right)
$$

where $\boldsymbol{q}_{\boldsymbol{o}}$ is the set of robot's nominal joint variables and $\Delta \boldsymbol{q}$ is the set of the compensation errors.

The second method which is the topic of this thesis is known as calibration. The calibration method corrects the robotic manipulator tip pose by correcting the links geometrical parameters in the kinematic model as shown in Equation 3.4 below.

$$
\boldsymbol{X}=f\left(\boldsymbol{q}_{o}, \boldsymbol{g}_{o}+\Delta \boldsymbol{g}\right)
$$

where $\boldsymbol{g}_{\boldsymbol{o}}$ is the set of the robot's nominal geometric parameters and $\Delta \boldsymbol{g}$ is the set of geometric errors of the robot. 
The kinematic modeling basis will be discussed in the following sections of this chapter. Then a full robotic tooling - vision system description will be provided which will help in building the final kinematic model for the calibration technique developed in this thesis.

\subsection{System Description}

A complete description of the system that was utilized for developing the calibration process is provided in this section. The system that was used as a test bed for verifying the calibration algorithm developed in this thesis is an automated percussive riveting system developed at Ryerson University [69]. The system consists of a 6 DOF ABB IRB4400 Robotic manipulator, a BIG 3D CREATOR vision system, a pneumatic rivet gun mounted on the robot's end-effector and finally a riveting jig. The rivet gun tool is mounted on the end-effector via a tool mount as shown in Figure 3-1 and 3-2 below. The robot system is also equipped with a camera system which is directly mounted on the robot's end-effector via a camera mount.

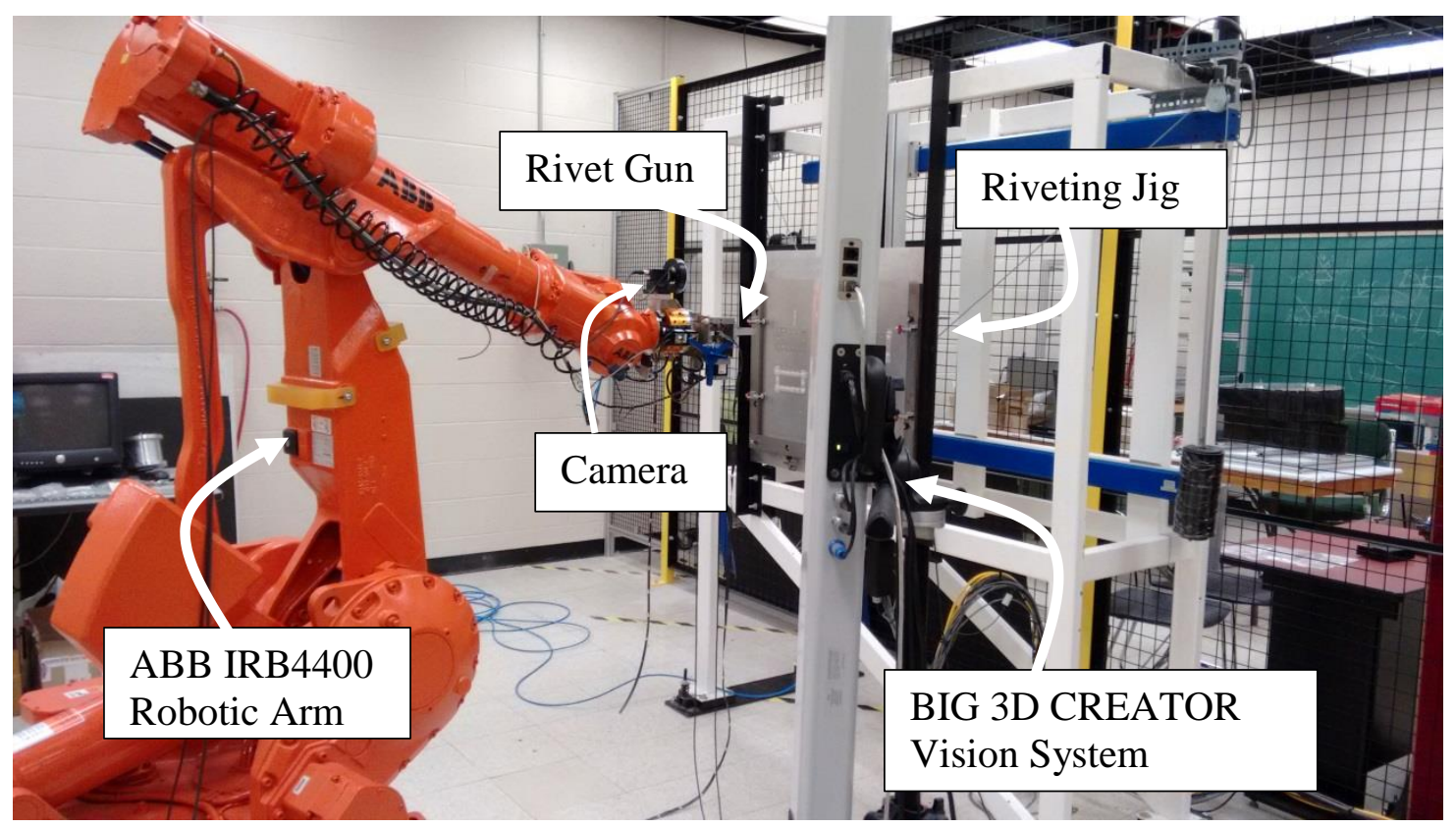

Figure 3-1: Automated Percussive Riveting System 


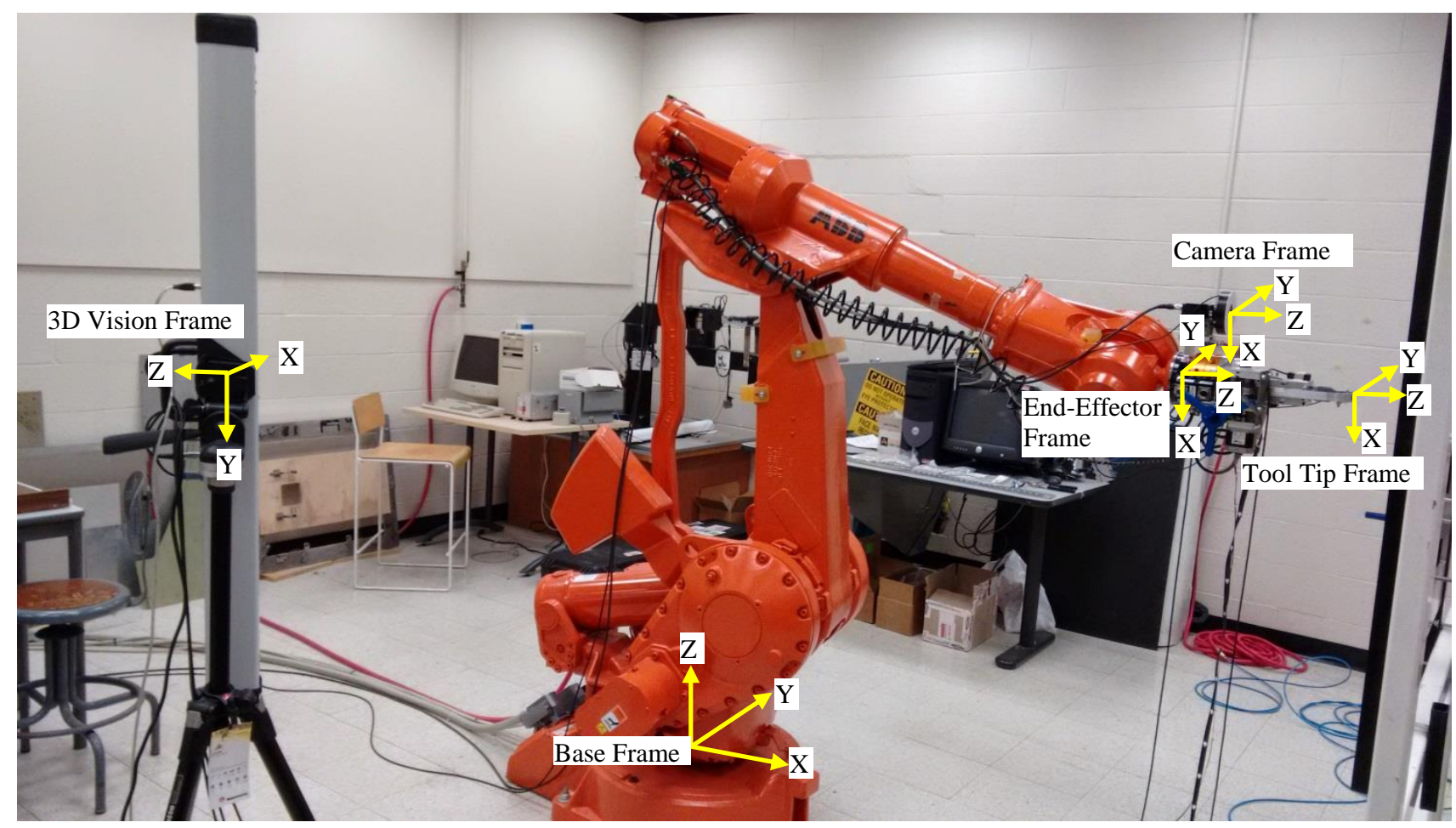

Figure 3-2: Robotic System with Frames

Multiple frames have to be defined to describe the whole riveting system. As shown in Figure 3-3, there are four frames that describe the main system. The first frame is the frame associated with the $3 \mathrm{D}$ vision system where the positive $\mathrm{Y}$ axis points towards the ground and the positive $\mathrm{Z}$ axis points towards the opposite direction of the CCD sensors. The rest of the frames are associated with the robot system where there is a robot base frame, end-effector frame, camera frame and tool frame. Both the camera and the tool tip frame have the same orientation as the end-effector frame. 


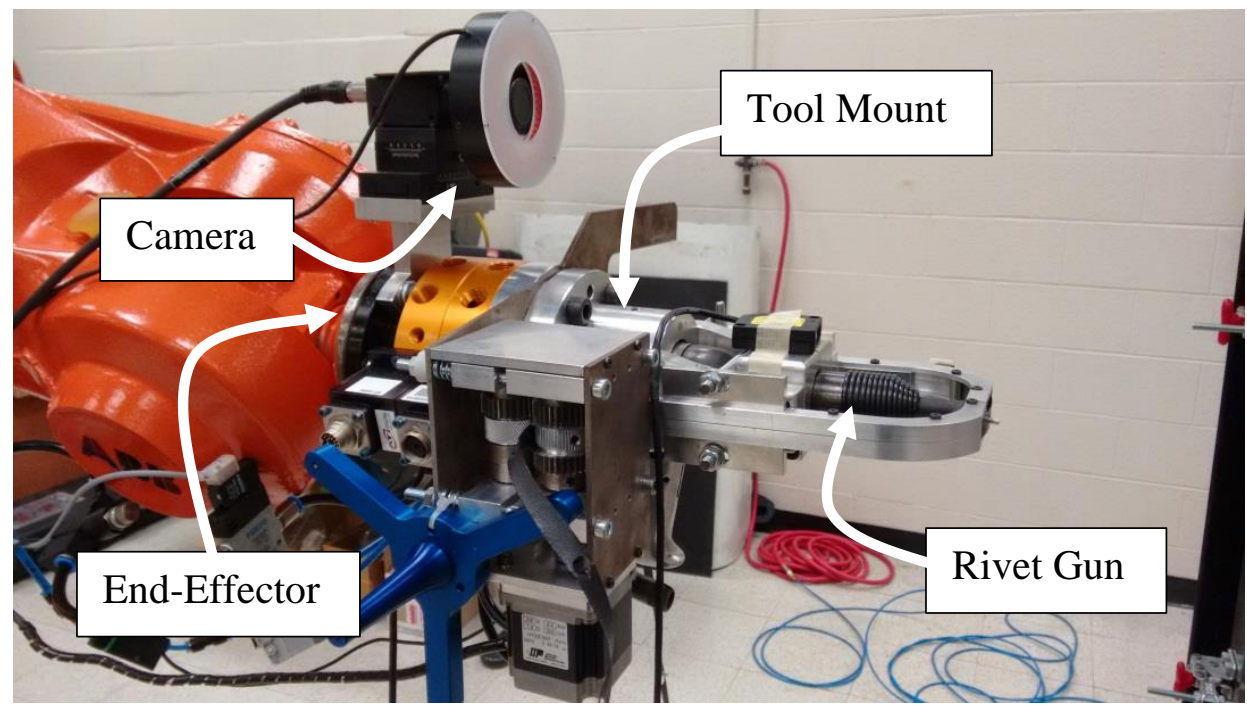

Figure 3-3: Robot Tool System

\subsection{Robot Kinematic Modelling with Tool}

\subsubsection{Position and Orientation}

Any general point given in space could be described with respect to a predetermined frame in form of a position vector as shown in Figure $3-4$. The position vector is a $3 \times 1$ column vector that has the coordinates' information of a point with respect to the origin of coordinate frame.

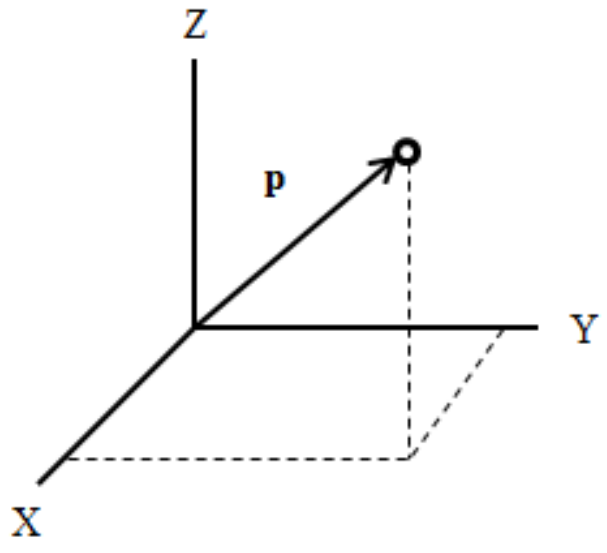

Figure 3-4: Position Vector 
The position vector does not contain enough information to describe the pose of the body in space. The missing information is the orientation of the body in space with respect to a predetermined frame. By attaching a coordinate frame to the body in a known configuration, the orientation of that frame could be expressed in terms of another frame using a rotation matrix. Rotation matrix is a $3 \times 3$ matrix that has the projection of each of the three axes of one frame on the three axes of the other frame.

Two of the main methods for explaining the orientation of one frame with respect to another frame will be explained. The first method using Euler angles which is a sequence of three successive rotations such that no two successive rotations are about the same axis. The second method using fixed axis rotation angles which is a sequence of three rotations about a fixed frame. The method which is going to be used in this thesis is Euler angles in the ZYX sequence.

The physical rotation of frame 1 to frame 2 is equivalent to the rotational matrix transformation from frame 2 to frame 1. The following equations show the derivation of the rotation matrix from frame 2 to frame 1 in details.

$$
\begin{aligned}
& \boldsymbol{R}\left(\theta_{Z}\right)=\left[\begin{array}{ccc}
\operatorname{Cos} \theta_{Z} & -\operatorname{Sin} \theta_{Z} & 0 \\
\operatorname{Sin} \theta_{Z} & \operatorname{Cos} \theta_{Z} & 0 \\
0 & 0 & 1
\end{array}\right] \\
& \boldsymbol{R}\left(\theta_{Y}\right)=\left[\begin{array}{ccc}
\operatorname{Cos} \theta_{Y} & 0 & \operatorname{Sin} \theta_{Y} \\
0 & 1 & 0 \\
-\operatorname{Sin} \theta_{Y} & 0 & \operatorname{Cos} \theta_{Y}
\end{array}\right] \\
& \boldsymbol{R}\left(\theta_{X}\right)=\left[\begin{array}{ccc}
1 & 0 & 0 \\
0 & \operatorname{Cos} \theta_{X} & -\operatorname{Sin} \theta_{X} \\
0 & \operatorname{Sin} \theta_{X} & \operatorname{Cos} \theta_{X}
\end{array}\right]
\end{aligned}
$$




$$
\begin{gathered}
\boldsymbol{R}=\boldsymbol{R}\left(\theta_{Z}\right) \boldsymbol{R}\left(\theta_{Y}\right) \boldsymbol{R}\left(\theta_{X}\right) \\
\boldsymbol{R}_{\mathbf{2}}^{\mathbf{1}}=\left[\begin{array}{ccc}
C Z C Y & C Z S Y S X-C X S Z & S Z S X+C Z C X S Y \\
C Y S Z & C Z C X+S Z S Y S X & C X S Z S Y-C Z S X \\
-S Y & C Y S X & C Y C X
\end{array}\right]
\end{gathered}
$$

where $\mathrm{C}$ and $\mathrm{S}$ stand for Cosine and Sine while $\mathrm{Z}, \mathrm{Y}$ and $\mathrm{X}$ stand for $\theta_{\mathrm{Z}}, \theta_{\mathrm{Y}}, \theta_{\mathrm{X}}$ respectively.

The position vector describes the position of one frame with respect to another; while, the rotation matrix describes the orientation of one frame with respect to another. Combined together we have a full pose description of a body with respect to a predetermined frame. The homogeneous transformation is a $4 \times 4$ matrix that is constructed by combining the rotation matrix with the position vector as shown in Equation 3.10 below,

$$
\boldsymbol{T}_{\mathbf{2}}^{\mathbf{1}}=\left[\begin{array}{cc}
\boldsymbol{R}_{\mathbf{2}_{3 \times \mathbf{3}}}^{\mathbf{1}} & \boldsymbol{P}_{\mathbf{3} \times \mathbf{1}} \\
0_{1 \times 3} & 1
\end{array}\right]
$$

\subsubsection{Calibration System Kinematic Modeling}

The kinematic model could be developed for the described system by relating each of the of the system's components to each other as shown in Figure 3-5 below. The first step of creating the kinematic model is by attaching frames to each component. The next step is determining the homogeneous transformations between the different components. 


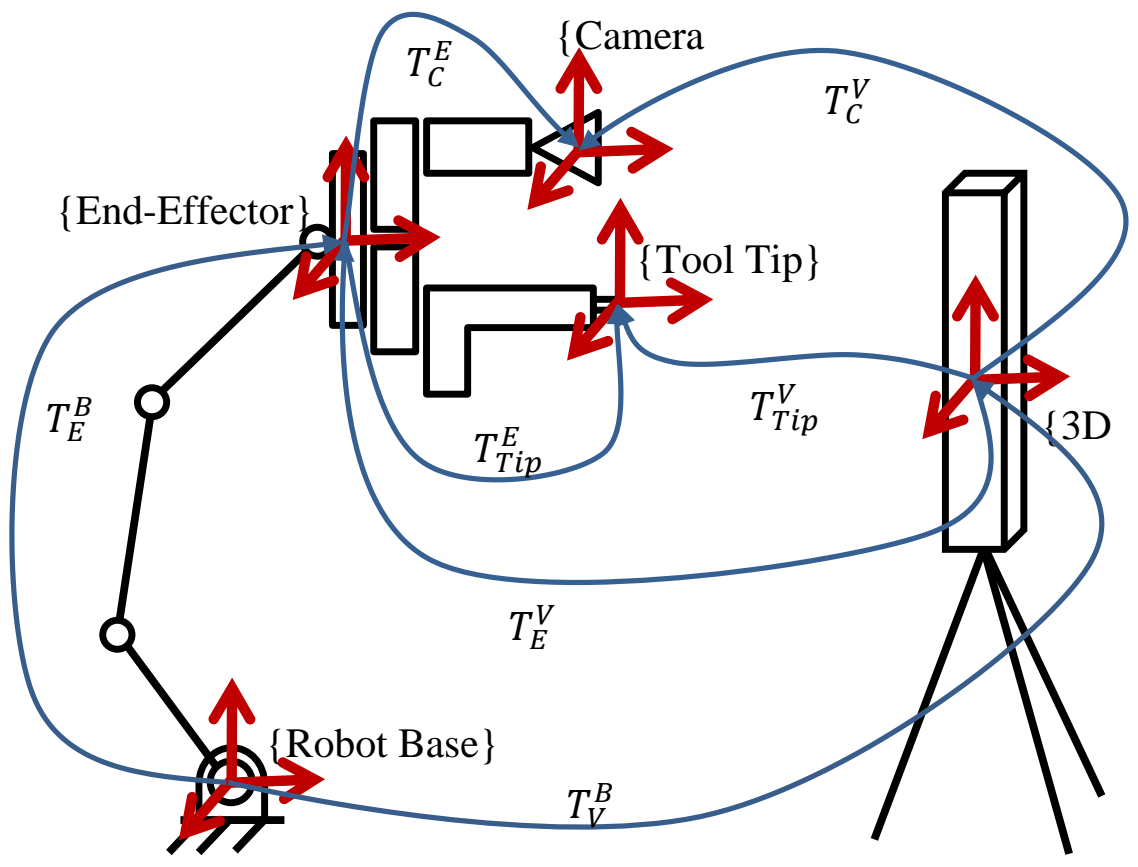

Figure 3-5: Robot - 3D Vision System Diagram

The pose of the end-effector as well as the pose of the tool (rivet gun) frame will be measured with respect to the $3 \mathrm{D}$ vision system using multiple fitting techniques which will be discussed in details in the following chapters. The nominal pose of the tool frame with respect to the end-effector frame could be easily determined from the design CAD models.

The tool (rivet gun) calibration will be the focus of this thesis. The tool could be described in terms of the robot's end-effector frame based on Figure 3-5 using Equations 3.11, 3.12 .

The homogeneous transformations shown in Figure 3-5 are defined as following,

- $\boldsymbol{T}_{\text {Tip }}^{V}$ : The homogeneous transformation from the tool tip frame to the 3D vision system frame which can be determined by the $3 \mathrm{D}$ vision system. 
- $\boldsymbol{T}_{\boldsymbol{E}}^{V}$ : The homogeneous transformation from the end-effector frame to the 3D vision system frame which can be determined by the 3D vision system.

- $\boldsymbol{T}_{\text {Tip }}^{E}$ : The homogeneous transformation from the tool tip frame to the end-effector frame which can be determined based on the $\boldsymbol{T}_{\text {Tip }}^{V}$ and $\boldsymbol{T}_{\boldsymbol{E}}^{V}$ transformations.

- $\boldsymbol{T}_{V}^{\boldsymbol{B}}$ : The homogeneous transformation from the 3D vision system frame to the base frame which can be obtained through alignment.

- $\boldsymbol{T}_{\boldsymbol{E}}^{\boldsymbol{B}}$ : The homogeneous transformation from the end-effector frame to the base frame which can be determined via the robot's controller (refer to Appendix D).

$$
\begin{gathered}
T_{T i p}^{V}=T_{E}^{V} T_{T i p}^{E} \\
T_{T i p}^{E}=\left(T_{E}^{V}\right)^{-1} T_{T i p}^{V}
\end{gathered}
$$

It could be also described in terms of the robot base frame using the following equation,

$$
\begin{aligned}
& T_{T i p}^{B}=T_{E}^{B} T_{T i p}^{E} \\
& T_{T i p}^{B}=T_{V}^{B} T_{T i p}^{V}
\end{aligned}
$$

Combining both Equations 3.13 and 3.14,

$$
T_{E}^{B} T_{T i p}^{E}=T_{V}^{B} T_{T i p}^{V}
$$

where the right-hand side of Equation 3.15 corresponds to the measured tip pose, while the lefthand side corresponds to the calibrated tip pose.

The transformation from the tool tip frame to the end-effector frame is the transformation which requires the calibration. The transformation from the end-effector frame to the vision 
system frame is known using the end-effector position estimation technique as described in Chapter 5 section 2. The transformation from the tool frame to the end-effector frame is also known using tool orientation estimation technique as described in Chapter 5 section 3.

\subsection{Error Modelling}

In this section, a model that relates the tip pose errors to the geometric links errors will be formulated. The formulated error model will be used in the next chapter to map the errors from the tip pose back to identifying the geometric links errors. The mapping methods will include both linear and nonlinear approaches.

As previously discussed, the tip pose $\boldsymbol{X}$ could be expressed as a nonlinear function of the links geometric parameters as well as the joint variables. In case of mounting a tool on the robot's end-effector, the tool tip pose is considered as an extension of the robot kinematic model. The tool tip pose could also be expressed as a nonlinear function of the robot joint variables, the robot's geometric parameters and the tool geometric parameters. Since the tool is mounted on the end-effector via a tool mount which is static, the tool would not include any joint variables. The following equation presents this relation,

$$
\boldsymbol{X}_{t_{o}}=f\left(\mathbf{q}, \boldsymbol{g}_{r}, \boldsymbol{g}_{t}\right)
$$

where $\boldsymbol{X}_{\boldsymbol{t}_{\boldsymbol{o}}}$ is the nominal tool tip pose, $\mathbf{q}$ is the robot's joint variables, $\boldsymbol{g}_{\boldsymbol{r}}$ is the nominal robot geometric parameters and $\boldsymbol{g}_{\boldsymbol{t}}$ is the nominal tool geometric parameters.

The forward kinematic model of the robot in addition to the mounted tool does not predict the precise position and orientation of the tool. The discrepancy between the actual tool 
pose and the kinematic tool pose is due to; first, the robot's links geometric errors; second, the tool/tool mount geometric errors as shown in the following equation,

$$
\boldsymbol{X}_{\boldsymbol{t}_{\boldsymbol{m}}}=f\left(\mathbf{q}, \boldsymbol{g}_{r}+\Delta \boldsymbol{g}_{r}, \boldsymbol{g}_{t}+\Delta \boldsymbol{g}_{t}\right)
$$

Where $\boldsymbol{X}_{\boldsymbol{t}_{\boldsymbol{m}}}$ is the measured tool tip pose, $\Delta \boldsymbol{g}_{\boldsymbol{r}}$ is the set of robot geometric link errors and $\Delta \boldsymbol{g}_{\boldsymbol{t}}$ is the set of the tool/tool mount geometric link errors.

Using both Equations 3.16 and 3.17, the tip pose errors could be determined by subtracting both equations. Equation 3.19 below shows that the tool tip position and orientation errors are a function of both the robot's geometric link errors and the tool's geometric link errors.

$$
\begin{gathered}
\Delta \boldsymbol{X}_{\boldsymbol{t}}=\boldsymbol{X}_{\boldsymbol{t}_{\boldsymbol{m}}}-\boldsymbol{X}_{\boldsymbol{t}_{\boldsymbol{o}}} \\
\Delta \boldsymbol{X}_{\boldsymbol{t}}=f\left(\mathbf{q}, \widehat{\boldsymbol{g}}_{r}, \boldsymbol{g}_{t}+\Delta \boldsymbol{g}_{t}\right)-f\left(\mathbf{q}, \widehat{\boldsymbol{g}}_{r}, \boldsymbol{g}_{t}\right)
\end{gathered}
$$

By assuming that the robotic manipulator was calibrated before the user attempts to mount the tool on the end-effector, the term $\boldsymbol{g}_{\boldsymbol{r}}+\Delta \boldsymbol{g}_{\boldsymbol{r}}$ is replaced by $\widehat{\boldsymbol{g}}_{\boldsymbol{r}}$ which is the calibrated robot geometric parameters. 


\section{CHAPTER 4: CALIBRATION ALGORITHM}

In the previous chapter, the system set up including the robotic manipulator, tool and the measurement system was described in details. Also in the previous chapter, an error model that describes the tool geometric errors as well as the tool pose errors captured by the measurement system was provided.

In this chapter, the methods of mapping the tip errors captured by the measurement system to the tool geometric errors will be presented. There are two approaches for mapping the tip errors to the tool geometric errors which are the linear approach and the nonlinear approach. The first section of this chapter discusses the linear approach and its validity for the tool calibration application. The non-linear formulation is subsequently presented in the second section of this chapter.

\subsection{Linear Formulation}

As explained in the previous chapters, the robot's end-effector position and orientation could be described as a function of the robotic manipulator geometric parameters $\boldsymbol{g}$ as well as the joint variables $\mathbf{q}$ as expressed in Equation 3.1.

Multiple methods could be used for producing the forward kinematics model for the robotic manipulator such as the Denavit-Hartenberg (DH) method or the six-parameter method as used in this thesis. The next step is the determination of the end-effector or tip infinitesimal pose change as a function of the infinitesimal change of the geometric parameters. This could be done by applying Taylor expansion as described by the following equation, 


$$
f\left(\mathbf{q}, \widehat{\boldsymbol{g}}_{r}, \boldsymbol{g}_{t}\right)=f\left(\mathbf{q}, \widehat{\boldsymbol{g}}_{r}, \boldsymbol{g}_{t_{o}}\right)+\frac{\partial f\left(\mathbf{q}, \widehat{\boldsymbol{g}}_{r}, \boldsymbol{g}_{t_{o}}\right)}{\partial \boldsymbol{g}_{t}} \times\left(\boldsymbol{g}_{t}-\boldsymbol{g}_{t_{o}}\right)+\boldsymbol{O}
$$

Ignoring the higher order terms $\boldsymbol{O}$ in the previous equation and reordering the terms will lead to the following,

$$
\Delta X_{t}=J_{t} \Delta g_{t}
$$

where $\boldsymbol{\Delta}_{\boldsymbol{t}}=\boldsymbol{X}_{\boldsymbol{t}_{\boldsymbol{m}}}-\boldsymbol{X}_{\boldsymbol{o}}$ is the difference between the actual (measured) $\boldsymbol{X}_{\boldsymbol{t}_{\boldsymbol{m}}}=$ $f\left(\mathbf{q}, \widehat{\boldsymbol{g}}_{r}, \boldsymbol{g}_{\boldsymbol{t}}\right)$ and nominal tip pose $\boldsymbol{X}_{\boldsymbol{o}}=f\left(\mathbf{q}, \widehat{\boldsymbol{g}}_{\boldsymbol{r}}, \boldsymbol{g}_{\boldsymbol{t}_{\boldsymbol{o}}}\right)$ which is the tip pose error.

$\Delta \boldsymbol{g}_{\boldsymbol{t}}=\boldsymbol{g}_{\boldsymbol{t}}-\boldsymbol{g}_{\boldsymbol{t}_{\boldsymbol{o}}}$ is the difference between the actual and nominal manipulator geometric parameters.

$J_{t}=\frac{\partial f\left(\mathbf{q}, \widehat{g}_{r}, g_{t_{0}}\right)}{\partial g_{t}}$ is the partial differentiation of the function with respect to the geometric parameters which is known as the robot's Jacobian matrix.

Equation 4.2 describes the relation between the tool geometric errors and the tip pose errors in a linear form. The mapping from the geometric errors to the tip errors is taking place through the Jacobian matrix. Hence, it is called the error mapping matrix. Furthermore, the calibration takes place by measuring the tip pose error and then mapping it back to the geometric tool errors. This could be achieved using the pseudo inverse of the Jacobian matrix also known as the Moore-Penrose formulation shown in the following equation,

$$
\Delta g_{t}=J^{+} \Delta X_{t}
$$

where $J^{+}=\left(J_{t}{ }^{T} J_{t}\right)^{-1} J_{t}{ }^{T}$ is the Jacobian pseudo inverse for an over-determined case. 
The calibration process starts by measuring the tip pose errors of the robot in multiple configurations (greater than the number of unknowns) and then calculating the pseudo inverse of the Jacobian matrix to determine the geometric tool errors.

\subsubsection{Eye-to-Hand vs. Eye-in-Hand}

There are two set-ups that could be used for determining the tip errors $\Delta \boldsymbol{X}_{\boldsymbol{t}}$. The first setup is when the measurement device is fixed on the workshop floor (space fixed frame) and totally separate from the manipulator that requires calibration. The manipulator arm has to be within the range of the measurement device capability such that the measurement device could accurately sense the robot tip pose errors. Contrary to the first set-up, the second set-up is when the measurement device is mounted on the manipulator arm (body fixed frame) which requires calibration. In this set-up the measurement device is not static but reflects the movement of the robot. The robot tip pose errors are captured by comparing the errors reflected in the measurements against an external known element. The first set-up is called eye-to-hand while the second set-up is called eye-in-hand.

The robotic system presented above includes both set-ups. The camera mounted on the robotic arm is an example of the second set-up which is the eye-in-hand. The eye-in-hand set-up is being used in the robotic tooling self-calibration algorithm developed and discussed in section 4.4.2. However, the 3D vision device is an example of the first set-up which is the eye-to-hand. This set-up is the focus of the technique developed in this thesis. 


\subsubsection{Geometric Errors}

There are six geometric errors parameters expressed by $\Delta \boldsymbol{g}$ in the following equation. The first three errors $\Delta \boldsymbol{x}, \Delta \boldsymbol{y}$ and $\Delta \boldsymbol{z}$ are the position errors. The last three errors $\delta \boldsymbol{x}, \delta \boldsymbol{y}$ and $\delta \boldsymbol{z}$ are the orientation (angles) errors.

$$
\Delta \boldsymbol{g}=[\Delta \boldsymbol{x} \Delta \boldsymbol{y} \Delta \mathbf{z} \delta \boldsymbol{x} \delta \boldsymbol{y} \delta \boldsymbol{z}]^{T}
$$

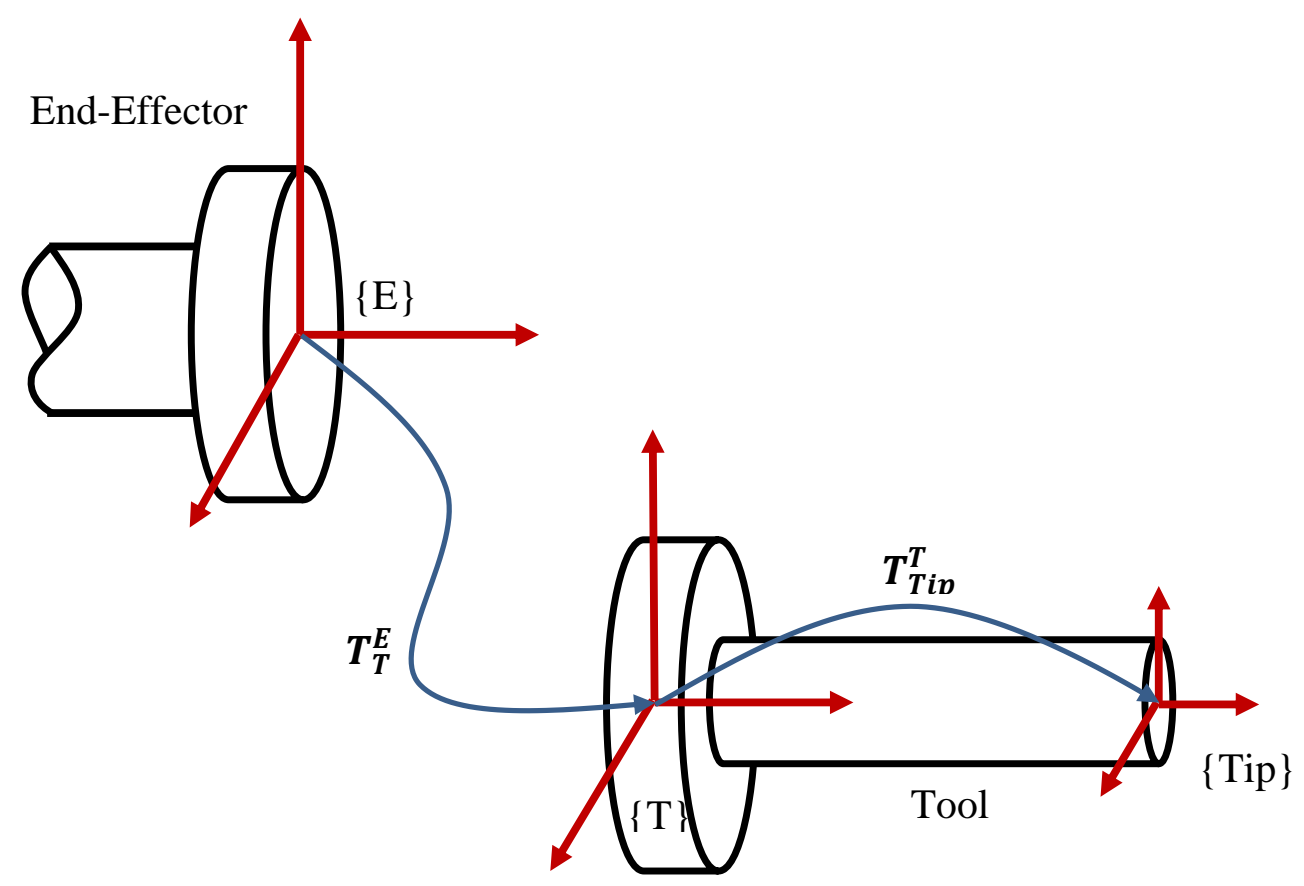

Figure 4-1: Tool - End-Effector Relation

Referring to Figure 4-1, the actual transformation from the tool frame to the end-effector frame could be expressed by,

$$
T_{\text {Tip }}^{E}=\left[\begin{array}{cc}
R_{T i p}^{E} & P_{T i p}^{E} \\
0 & 1
\end{array}\right]
$$

The transformation from the tool tip frame to the end-effector frame could be divided into two transformations as expressed using the following equations, 


$$
\begin{gathered}
\boldsymbol{T}_{\text {Tip }}^{E}=\boldsymbol{T}_{T}^{E} \boldsymbol{T}_{\text {Tip }}^{T} \\
\boldsymbol{T}_{\text {Tip }}^{E}=\left[\begin{array}{cc}
\boldsymbol{R}_{T}^{E} & \boldsymbol{P}_{T}^{E} \\
0 & 1
\end{array}\right]\left[\begin{array}{cc}
\boldsymbol{R}_{\text {Tip }}^{T} & \boldsymbol{P}_{\text {Tip }}^{T} \\
0 & 1
\end{array}\right]=\left[\begin{array}{cc}
\boldsymbol{R}_{T}^{E} \boldsymbol{R}_{\text {Tip }}^{T} & \boldsymbol{R}_{T}^{E} \boldsymbol{P}_{\text {Tip }}^{T}+\boldsymbol{P}_{T}^{E} \\
0 & 1
\end{array}\right]
\end{gathered}
$$

By equating Equation 4.5 and Equation 4.7,

$$
R_{T i p}^{E}=R_{T}^{E} R_{T i p}^{T} \quad P_{T i p}^{E}=R_{T}^{E} P_{T i p}^{T}+P_{T}^{E}
$$

where the orientation errors $\delta \boldsymbol{x}, \delta \boldsymbol{y}$ and $\delta \boldsymbol{z}$ are introduced in the rotation matrix $\boldsymbol{R}_{\boldsymbol{T}}^{\boldsymbol{E}}$ while the position errors $\Delta \boldsymbol{x}, \Delta \boldsymbol{y}$ and $\Delta \boldsymbol{z}$ are introduced in the position vector $\boldsymbol{P}_{\boldsymbol{T}}^{E}$.

As noticed from Equation 4.8 that the rotation matrix $\boldsymbol{R}_{\text {Tip }}^{E}$ is solely affected by the orientation errors. On the other hand, the position vector $\boldsymbol{P}_{\text {Tip }}^{E}$ is affected by both the orientation errors introduced in $\boldsymbol{R}_{\boldsymbol{T}}^{\boldsymbol{E}}$ in addition to the position errors introduced in $\boldsymbol{P}_{\boldsymbol{T}}^{\boldsymbol{E}}$.

\subsubsection{Jacobian Matrix}

The Jacobian matrix is driven from the homogeneous transformation of the tool tip frame to the end-effector frame as expressed in the following equations,

$$
\begin{gathered}
\boldsymbol{T}_{\text {Tip }}^{T}=\left[\begin{array}{cc}
\boldsymbol{R}_{\text {Tip }}^{T} & \boldsymbol{P}_{\text {Tip }}^{T} \\
0 & 1
\end{array}\right] \\
\boldsymbol{R}_{T}^{E}=\left[\begin{array}{lll}
\boldsymbol{n}_{1} & \boldsymbol{o}_{1} & \boldsymbol{a}_{1}
\end{array}\right]
\end{gathered}
$$

where $\boldsymbol{n}_{\mathbf{1}}, \boldsymbol{o}_{\mathbf{1}}$ and $\boldsymbol{a}_{\mathbf{1}}$ are the columns of the rotation matrix. Then the Jacobian matrix for one link (i.e. two links in the robotic tooling self-calibration algorithm), 


$$
\begin{gathered}
\mathrm{J}_{E}= \\
{\left[\begin{array}{cccccc}
n_{1} & o_{1} & a_{1} & n_{1} \times\left(P_{\text {Tip }}^{E}-P_{T}^{E}\right) & o_{1} \times\left(P_{\text {Tip }}^{E}-P_{T}^{E}\right) & a_{1} \times\left(P_{\text {Tip }}^{E}-P_{T}^{E}\right) \\
0 & 0 & 0 & n_{1} & o_{1} & a_{1}
\end{array}\right]}
\end{gathered}
$$

Since everything has to be expressed in terms of the robot's base frame, the tool to endeffector transformation has to be multiplied by the end-effector to the base transformation as shown in the following equations,

$$
T_{T i p}^{B}=T_{E}^{B} T_{T i p}^{E}
$$

By expanding and multiplying both transformations,

$$
\begin{aligned}
\boldsymbol{T}_{\text {Tip }}^{B} & =\left[\begin{array}{cc}
\boldsymbol{R}_{E}^{B} & \boldsymbol{P}_{E}^{B} \\
0 & 1
\end{array}\right]\left[\begin{array}{cc}
\boldsymbol{R}_{\text {Tip }}^{E} & \boldsymbol{P}_{\text {Tip }}^{E} \\
0 & 1
\end{array}\right] \\
\boldsymbol{T}_{\text {Tip }}^{B} & =\left[\begin{array}{cc}
\boldsymbol{R}_{E}^{B} \boldsymbol{R}_{\text {Tip }}^{E} & \boldsymbol{R}_{E}^{B} \boldsymbol{P}_{\text {Tip }}^{E}+\boldsymbol{P}_{E}^{B} \\
0 & 1
\end{array}\right]
\end{aligned}
$$

From Equations 4.12, 4.13, 4.14 above, the Jacobian matrix expressed in terms of the base frame could be shown as following,

$$
\begin{gathered}
\mathrm{J}_{t}= \\
{\left[\begin{array}{cccccc}
\boldsymbol{R}_{E}^{B}\left[n_{1}\right. & o_{1} & \left.a_{1}\right] & R_{E}^{B}\left[n_{1} \times\left(P_{\text {Tip }}^{E}-P_{T}^{E}\right)\right. & o_{1} \times\left(P_{T i p}^{E}-P_{T}^{E}\right) & a_{1} \times\left(P_{T i p}^{E}-P_{T}^{E}\right) \\
0 & 0 & 0 & R_{E}^{B}\left[n_{1}\right. & o_{1} & \left.a_{1}\right]
\end{array}\right]}
\end{gathered}
$$

$\mathbf{J}_{t}$ can be factorized into the following matrices,

$$
\begin{aligned}
& \mathbf{J}_{t}=\left[\begin{array}{cc}
\boldsymbol{R}_{E}^{B} & 0 \\
0 & \boldsymbol{R}_{E}^{B}
\end{array}\right] \mathbf{J}_{E} \\
& \mathbf{J}_{\boldsymbol{t}}=\operatorname{diag}\left(\boldsymbol{R}_{E}^{\boldsymbol{B}}\right) \mathbf{J}_{E}
\end{aligned}
$$

For the sake of notation simplification, the following notation is used, 


$$
\mathfrak{R}_{E}^{B}=\operatorname{diag}\left(\boldsymbol{R}_{E}^{B}\right)
$$

By substituting Equation 4.18 in equation 4.2 for $n$ number of measurements,

$$
\begin{gathered}
{\left[\begin{array}{c}
\Delta \boldsymbol{X}_{\boldsymbol{t}_{1}} \\
\Delta \boldsymbol{X}_{\boldsymbol{t}_{2}} \\
\vdots \\
\Delta \boldsymbol{X}_{\boldsymbol{t}_{n}}
\end{array}\right]=\left[\begin{array}{c}
\mathbf{J}_{\boldsymbol{t}_{1}} \\
\mathbf{J}_{t_{2}} \\
\vdots \\
\mathbf{J}_{t_{n}}
\end{array}\right] \Delta \boldsymbol{g}_{\boldsymbol{t}}} \\
\Delta \boldsymbol{X}_{\boldsymbol{A}}=\left[\begin{array}{c}
\mathfrak{R}_{E_{1}}^{B} \mathbf{J}_{E} \\
\mathfrak{R}_{E_{2}}^{B} \mathbf{J}_{E} \\
\vdots \\
\mathfrak{R}_{E_{n}}^{B} \mathbf{J}_{E}
\end{array}\right] \Delta \boldsymbol{g}_{\boldsymbol{t}}
\end{gathered}
$$

where $\Delta \boldsymbol{X}_{\boldsymbol{A}}$ is the augmented tip errors. Since $\mathbf{J}_{\boldsymbol{E}}$ is always constant regardless of the robot pose because the tool/tool mount are fixed, the following simplification could be done,

$$
\left[\begin{array}{c}
\mathfrak{R}_{E_{1}}^{B} \mathbf{J}_{E} \\
\mathfrak{R}_{E_{2}}^{B} \mathbf{J}_{E} \\
\vdots \\
\mathfrak{R}_{E_{n}}^{B} \mathbf{J}_{E}
\end{array}\right]=\left[\begin{array}{c}
\mathfrak{R}_{E_{1}}^{B} \\
\mathfrak{R}_{E_{2}}^{B} \\
\vdots \\
\mathfrak{R}_{E_{2}}^{B}
\end{array}\right] \mathbf{J}_{E}=A \mathbf{J}_{E}
$$

$\mathbf{J}_{\boldsymbol{E}}$ is a $6 \mathrm{x} 6$ matrix and $\boldsymbol{A}$ is a $6 \mathrm{nx} 6$ matrix of $\mathfrak{R}_{\boldsymbol{E}}^{\boldsymbol{B}}$ for n measurements. where $\mathrm{n}$ is the number of measurements. Substitute into Equation 4.3,

$$
\Delta g_{t}=\left(J_{E}{ }^{T} A^{T} A J_{E}\right)^{-1} J_{E}{ }^{T} A^{T} \Delta X_{A}
$$

where $\boldsymbol{A}^{T} \boldsymbol{A}=\boldsymbol{I}$.

$$
\operatorname{rank}\left(\boldsymbol{J}_{E}{ }^{T} \boldsymbol{J}_{E}\right)=\operatorname{rank}\left(\boldsymbol{J}_{E}\right) \leq \mathbf{6}
$$


As shown in Equations 4.23, the Jacobian matrix could be full rank since the size of the matrix is $6 \times 6$. Hence, the whole set of the tool geometric parameters could be identified. The next analysis step is checking the sensitivity of the Jacobian matrix to the input parameters while mapping the errors to the output parameters. In other words, a check of how the Jacobian matrix is prone to the measurement noise for determining the tool link parameters.

The condition number of the Jacobian matrix is a measure of the sensitivity or impact of a small change in the input parameters on the output parameters. The condition number value is limited to have a value greater or equal one. A matrix with a small condition number is known as a well-conditioned matrix where it is more robust and less sensitive to the noise. On the other hand, a matrix with large condition number is known as an ill-conditioned matrix where it is more prone to the noise.

From Equation 4.12 above, the Jacobian matrix driven is based on the homogeneous transformation from the end-effector frame to the tool tip frame. Since the three frames in Figure 4-1 have the same orientation; the Jacobian matrix is expected to be a sparse matrix in which most of its elements are zero. Moreover, the tool tip frame is merely a translation from the tool frame by the tool length value in the $\mathrm{Z}$ direction. Hence, the cross multiplication portion of the Jacobian matrix will lead to a Jacobian matrix with most of its elements are zero and few elements with a large value (tool length) (refer to Appendix E). Given the tool parameters, the condition number for the given Jacobian matrix is in the order of $10^{5}$.

A scaling factor is introduced for calculating the Jacobian matrix in order to minimize the value of the condition number. The scaling factor used is the normalization of the position vector used for the cross multiplication as shown in the equation below, 


$$
P_{\text {Tip }}^{E}{ }_{\text {norm }}=\frac{P_{\text {Tip }}^{E}}{\left\|P_{\text {Tip }}^{E}\right\|}
$$

By normalizing the position vector, the condition number of the Jacobian matrix was reduced from the order of $10^{5}$ to the approximate value of 2.6.

\subsection{Nonlinear Formulation}

Contrary to the linear formulation, the nonlinear formulation uses the forward kinematics model for the robot directly. The tool geometric errors are estimated and inserted in the forward kinematics equation to determine the tip pose. Using the difference between the estimated pose and the measured pose, the links geometric errors are adjusted for the subsequent iterations to approach the measured pose as given in the equation below,

$$
\boldsymbol{X}_{\boldsymbol{t}}=f\left(\mathbf{q}, \widehat{\boldsymbol{g}}_{\boldsymbol{r}}, \boldsymbol{g}_{\boldsymbol{t}}+\Delta \boldsymbol{g}_{\boldsymbol{t}}\right) \rightarrow \boldsymbol{X}_{\boldsymbol{t}_{m}}
$$

The estimation of the geometric link errors is achieved in this thesis using a search algorithm technique. Genetic Algorithm was used as the search technique for determining the geometric link errors based on the difference between the estimated pose and the actual (measured) pose as a fitness function. The GA's objective is to minimize the fitness function to approach zero and derive the accuracy of the estimated values below the required value.

$$
\left\|X_{t_{m}}-X_{t_{o}}\right\|_{2}<\mathrm{e}
$$

Where, $\left\|\boldsymbol{X}_{\boldsymbol{t}_{\boldsymbol{m}}}-\boldsymbol{X}_{\boldsymbol{t}_{\boldsymbol{o}}}\right\|_{2}$ is the Euclidean norm of the pose error and e is the required accuracy. 


\subsubsection{Genetic Algorithm}

Genetic Algorithm is an optimization technique which is traced back to the biological theory of natural evolution. Genetic Algorithm was first introduced by Holland in his book "Adaptation in natural and artificial systems" [70]. He explained the method of using the natural selection principals in optimization problems and constructed the first Genetic Algorithm.

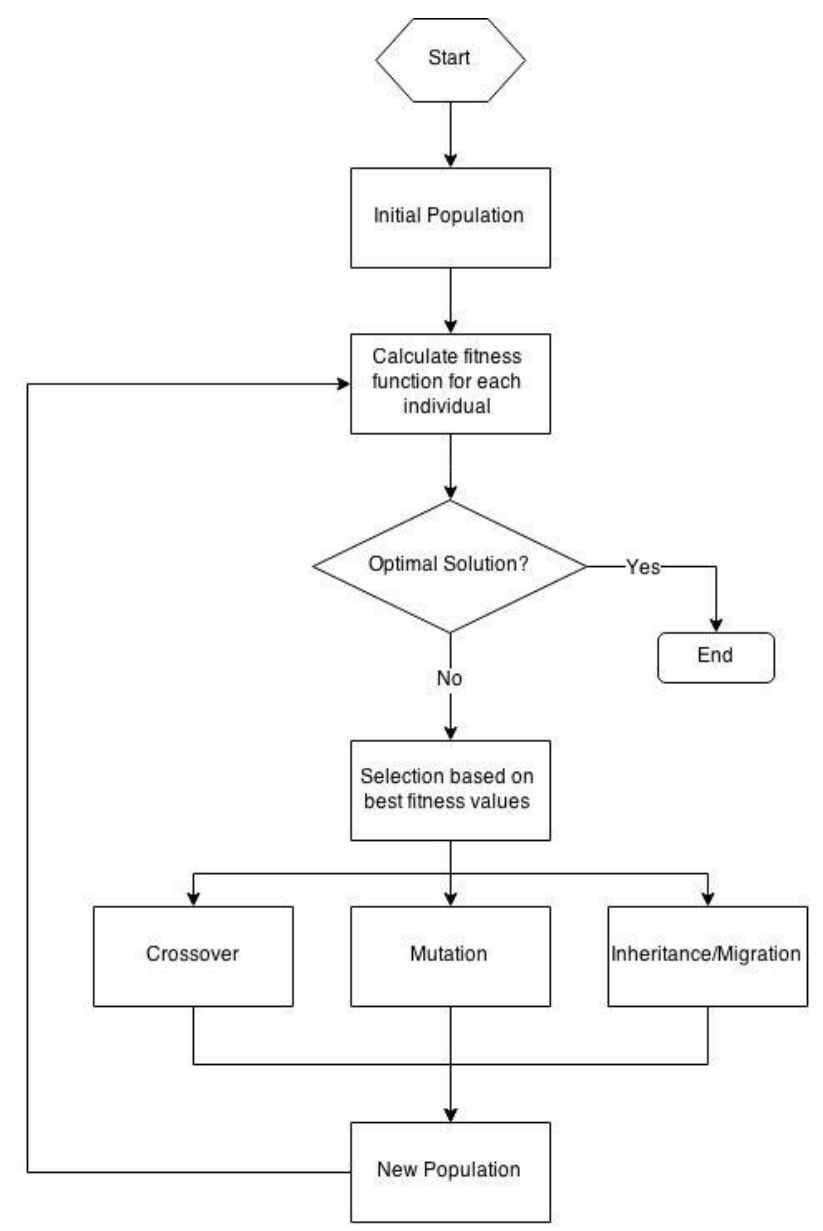

Figure 4-2: Genetic Algorithm

Genetic Algorithm is a stochastic search method for optimization of multivariable nonlinear systems. Genetic Algorithm is considered to a very popular search technique due to its 
robustness in finding an optimal solution. GA is a very simple and effective technique that uses the concept of "survival of the fittest" to optimize the results obtained at each generation.

The GA is process is shown in the flowchart in Figure 4-2 above. The algorithm starts by generating a random population for a specific number of individuals set by the user. The population is evaluated using a fitness function designed by the user to be minimized or maximized. The next step determines the elimination process of the least fit individuals of the population by applying the selection operation based on the fitness values. The fitness values act

as a weighting factor for each individual which determines the likelihood of the selection of individuals for next generation. The following step is applying three operations to the selected individuals from previous population which are crossover, mutation and inheritance/migration. The three operations create a new population with better qualities than the previous population. The evolved population is used as another generation which will be evaluated using the fitness function. The termination condition of the algorithm depends on the number of generations determined by the user or by the best fit (optimal solution) value.

\subsubsection{Fitness Function}

Formulating the fitness function is the crucial step which determines the success of the GA in finding an optimal solution. The fitness function must provide a result or a cost for comparing between multiple individual. Regarding the calibration problem, the fitness function should minimize the errors between the full pose of the tool based on the kinematic model and the measured full pose. The kinematic model provides the nominal pose of the tool which requires correction to be as close as possible to the measured pose as expressed in the equations, 


$$
\begin{gathered}
\min (f(x)) \\
f(x)=\left\|\boldsymbol{X}_{\boldsymbol{t}_{\boldsymbol{m}}}-\boldsymbol{X}_{\boldsymbol{t}_{\boldsymbol{o}}}\right\|_{2}
\end{gathered}
$$

where $\boldsymbol{X}_{\boldsymbol{t}_{\boldsymbol{m}}}$ is the measured tool pose and $\boldsymbol{X}_{\boldsymbol{t}_{\boldsymbol{o}}}$ is the nominal tool pose. The Euclidean norm of the difference between the measured pose and the nominal pose is the fitness function which is required to be minimized. The tool pose could be expressed by the following equation,

$$
\left\|X_{t_{m}}-X_{t_{o}}\right\|_{2}=\left\|P_{t_{m}}-P_{t_{o}}\right\|_{2}+\left\|R_{t_{m}}-R_{t_{o}}\right\|_{2}
$$

where $\left\|\boldsymbol{P}_{\boldsymbol{t}_{\boldsymbol{m}}}-\boldsymbol{P}_{\boldsymbol{t}_{\boldsymbol{o}}}\right\|_{2}$ is the Euclidean norm of the difference between the measured and nominal position vectors, while $\left\|\boldsymbol{R}_{\boldsymbol{t}_{\boldsymbol{m}}}-\boldsymbol{R}_{\boldsymbol{t}_{\boldsymbol{o}}}\right\|_{2}$ is the Euclidean norm of the difference between the measured and nominal rotation matrices.

There are three main approaches for performing the calibration process which are the position only calibration, the orientation only calibration and the full pose calibration. The fitness function was slightly adjusted for being reduced into a position only fitness function or an orientation only fitness function. This was achieved by adding weighting factors to each part of the equation as shown below,

$$
\left\|\boldsymbol{X}_{t_{\boldsymbol{m}}}-\boldsymbol{X}_{\boldsymbol{t}_{\boldsymbol{o}}}\right\|_{2}=w_{1} \times\left\|\boldsymbol{P}_{t_{\boldsymbol{m}}}-\boldsymbol{P}_{\boldsymbol{t}_{\boldsymbol{o}}}\right\|_{2}+w_{2} \times\left\|\boldsymbol{R}_{\boldsymbol{t}_{\boldsymbol{m}}}-\boldsymbol{R}_{\boldsymbol{t}_{\boldsymbol{o}}}\right\|_{2}
$$

The weighting factors could either take the value of one or zero. If the weighting factor $w_{1}$ is set to one while the weighting factor $w_{2}$ is set to zero, the fitness function will be reduced to a position only fitness function and will completely exclude the orientation part. However, if the $w_{1}$ is set to zero and $w_{2}$ is set to one, the fitness function represents an orientation only 
problem. If both $w_{1}$ and $w_{2}$ are set to one, the fitness function incorporates both position and orientation calibration.

\subsection{Calibration Formulation}

For each generation in the GA, the nominal position vector and rotation matrix will be updated by the value of the estimated geometric errors as expressed in the following equation,

$$
\boldsymbol{X}_{\boldsymbol{t}_{\boldsymbol{o}}}=f\left(\mathbf{q}, \widehat{\boldsymbol{g}}_{\boldsymbol{r}}, \boldsymbol{g}_{\boldsymbol{t}}+\Delta \boldsymbol{g}_{t}\right)
$$

The updated nominal pose $\boldsymbol{X}_{\boldsymbol{t}_{\boldsymbol{o}}}$ will then be inserted into the fitness function for evaluating each individual within the population. A generation of a new population will be evolved by applying the GA operations.

A tool calibration process has been developed based on the kinematic model developed in this chapter which is described by the flowchart shown in Figure 4-3 below. The calibration process starts by obtaining the robot's end-effector circular shaped data points as well as the tool (rivet gun) cylinder shaped data points which are described in details in Chapter 5. The second step is determining the position of the end-effector and the orientation of the rivet gun. The homogeneous transformation from the end-effector frame to the 3D vision system frame is calculated. The following step is the calculation of the transformation from the gun frame to the end-effector frame based on the measurements as well as the nominal parameters. Finally, the GA will estimate the errors via the fitness function. 


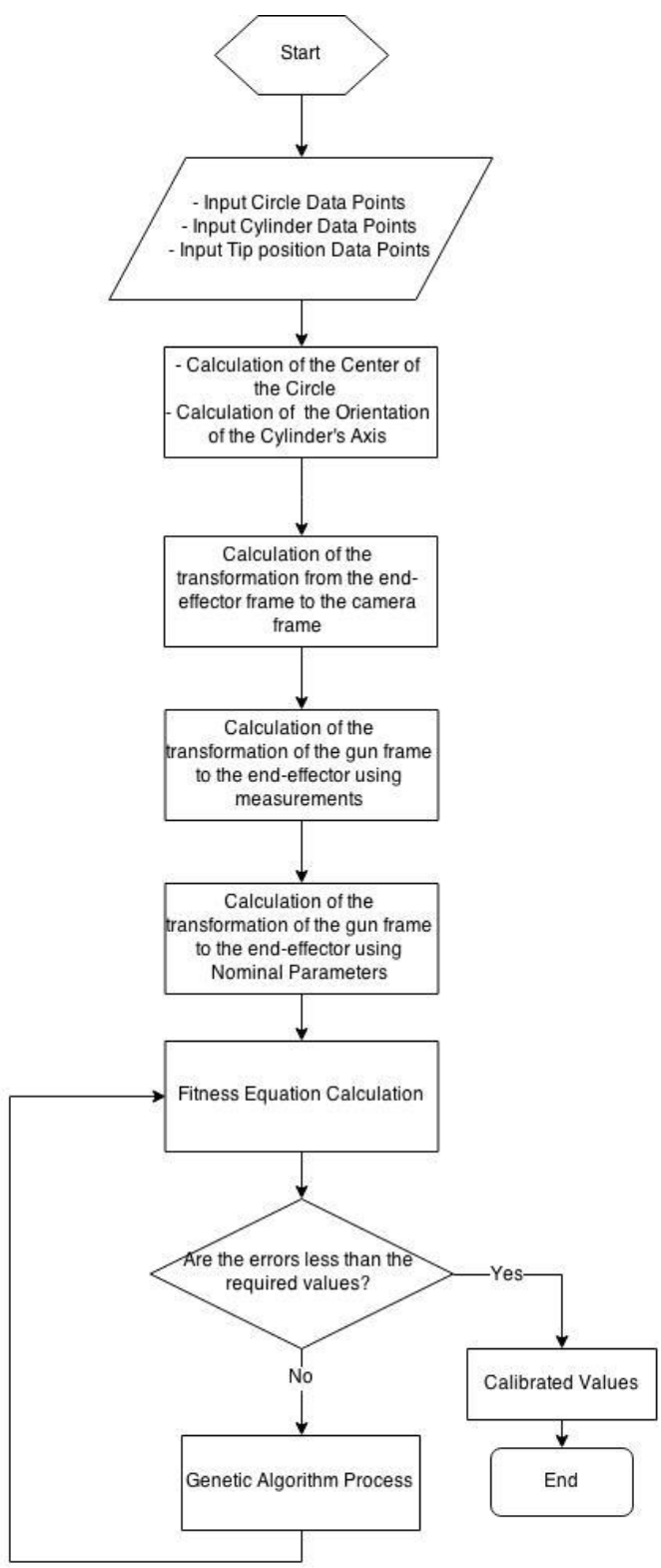

Figure 4-3: Calibration Algorithm 


\subsection{On-line vs. Off-line Error Measurement}

A robotic manipulators job is to repeatedly perform specific tasks on the workshop floor. There are two main methods for obtaining the tool tip errors $\Delta \boldsymbol{X}_{\boldsymbol{t}}$. The first is the on-line error measurement technique while the second is the off-line error measurement.

The on-line error measurement requires the movement of the robot for the formulation of the error model against pre-measured targets such as holes. In other words, the errors are only determined when the robot changes its pose via an eye-in-hand set-up. However, the off-line method does not require the movement of the robot for formulating the error model. The error could be determined based on a static robot pose via an eye-to-hand set-up.

\subsubsection{On-line Error Measurement for Robots}

The root idea of the self-calibration formulation in which the robotic tooling calibration is based on is setting the robot in multiple configurations. By moving the robot to multiple configurations to measure the distances between holes, the Jacobian matrices will be different. Different Jacobian matrices used in the formulation will allow the mapping of the distances errors to the geometric links errors.

For the calibration process to take place, the robot has to be moved such that each hole on the jig is within the field of vision of the measuring device (i.e. camera). Hence, the errors measurements have to be obtained while the robot is in operation. In this scenario, the errors measurements is said to be on-line since the robot is operating/on-line. 
According to the self-calibration model proposed by Gong et al. [53] the whole model is based on the concept that the robot should have different configurations for measuring multiple targets. The purpose behind this idea is having different Jacobian matrices as following,

$$
\Delta X_{C}=J_{C} \Delta g_{C}
$$

where $\Delta \boldsymbol{X}_{\boldsymbol{C}}$ is the set of sensor (camera) position errors, $\Delta \boldsymbol{g}_{\boldsymbol{C}}$ is the set of links geometric errors.

By measuring two different targets the equation above could be written as follows,

$$
\begin{aligned}
& X_{C_{i}}^{\prime}-X_{C_{i}}=J_{C i} \Delta g_{C} \\
& X_{C_{j}}^{\prime}-X_{C_{j}}=J_{C j} \Delta g_{C}
\end{aligned}
$$

where

- $\boldsymbol{X}_{\boldsymbol{C}_{\boldsymbol{i}}}$ is the first target measured position

- $\quad \boldsymbol{X}_{\boldsymbol{C}_{\boldsymbol{j}}}$ is the second target measured position

- $\quad \boldsymbol{X}_{\boldsymbol{C}_{\boldsymbol{i}}}^{\prime}$ is the first target actual position

- $\quad \boldsymbol{X}_{\boldsymbol{C}_{\boldsymbol{j}}}^{\prime}$ is the second target actual position

By subtracting Equations 4.33 and 4.34, the following equation could be obtained,

$$
X_{C_{i}}^{\prime}-X_{C_{j}}^{\prime}=X_{C_{i}}-X_{C_{j}}+\left(J_{C_{i}}-J_{C_{j}}\right) \Delta g_{C}
$$

By squaring both sides of the equation, 


$$
\begin{aligned}
\left\|X_{C_{i}^{\prime}}^{\prime}-X_{C_{j}}^{\prime}\right\|^{2} & \\
& =\left\|X_{C_{i}}-X_{C_{j}}\right\|^{2}+2\left(X_{C_{i}}-X_{C_{j}}\right)^{T}\left(J_{C_{i}}-J_{C_{j}}\right) \Delta g_{C} \\
& +\left\|\left(J_{C_{i}}-J_{C_{j}}\right) \Delta g_{C}\right\|^{2}
\end{aligned}
$$

Assuming $\Delta \boldsymbol{g}_{C}$ is small, and then we could ignore the second order term of $\Delta \boldsymbol{g}_{C}$,

$$
\left\|X_{C_{i}}^{\prime}-X_{C_{j}^{\prime}}^{\prime}\right\|^{2}-\left\|X_{C_{i}}-X_{C_{j}}\right\|^{2}=2\left(X_{C_{i}}-X_{C_{j}}\right)^{T}\left(J_{C_{i}}-J_{C_{j}}\right) \Delta g_{C}
$$

Comparing this to the following form,

$$
\Delta X_{C_{i j}}=\boldsymbol{G}_{i j} \Delta \boldsymbol{g}_{C}
$$

where

$$
\begin{gathered}
\Delta X_{C_{i j}}=\left\|X_{C_{i_{i}}}^{\prime}-X_{C_{i_{j}}}{ }^{\prime}\right\|^{2}-\left\|X_{C_{i_{i}}}-X_{C_{i_{j}}}\right\|^{2} \\
G_{i j}=2\left(X_{C_{i_{i}}}-X_{C_{i_{j}}}\right)^{T}\left(J_{C_{i}}-J_{C_{j}}\right)
\end{gathered}
$$

where $\left\|\boldsymbol{X}_{\boldsymbol{C}_{\boldsymbol{i}}}^{\prime}-\boldsymbol{X}_{\boldsymbol{C}_{j}}^{\prime}\right\|^{2}$ is the actual distance between the two targets measured accurately using CMM and $\left\|\boldsymbol{X}_{\boldsymbol{C}_{\boldsymbol{i}}}-\boldsymbol{X}_{\boldsymbol{C}_{\boldsymbol{j}}}\right\|^{2}$ is the calculated distance between the two targets using on-line measurement technique via the robot-mounted camera.

Solving for the unknowns (links parameters errors),

$$
\Delta \boldsymbol{g}_{C}=\boldsymbol{G}_{i j}^{+} \Delta X_{C_{i j}}
$$

where $\boldsymbol{G}_{i j}{ }^{+}$is the pseudo-inverse of $\boldsymbol{G}_{\boldsymbol{i}}$, 


$$
G_{i j}^{+}=\left(G^{T} G\right)^{-1} G^{T}
$$

\subsubsection{On-line Error Measurement for Robotic Tooling Calibration}

The self-calibration model developed by Gong et al. [53] is the core technique used in the tooling self-calibration algorithm. The self-calibration system set up is composed of a robotic arm which requires calibration, an end-effector mounted sensor and a jig with number of holes. The sensor mounted on the end-effector can determine the center of any hole within its field of vision. The robotic arm places the sensor in front of the hole where the center's position is measured with respect to the robot's base frame. The process is repeated for all the holes on the jig. The distances between the holes' centers are accurately measured using a CMM machine. An error formulation is developed to compare the distances calculated using the robot - sensor system and the distances obtained using the CMM machine. The error formulation is used to calibrate the geometric parameters of the robot's links.

The same method was implemented in the robotic tooling self-calibration paper [74]; however, it was only used for calibrating the tool mounted on the robot instead of calibrating the robot itself. A new formulation had to be developed for applying the self-calibration technique for calibrating the tool. The system used for performing the robotic tooling self-calibration process is shown in Figure 4-4 below. 


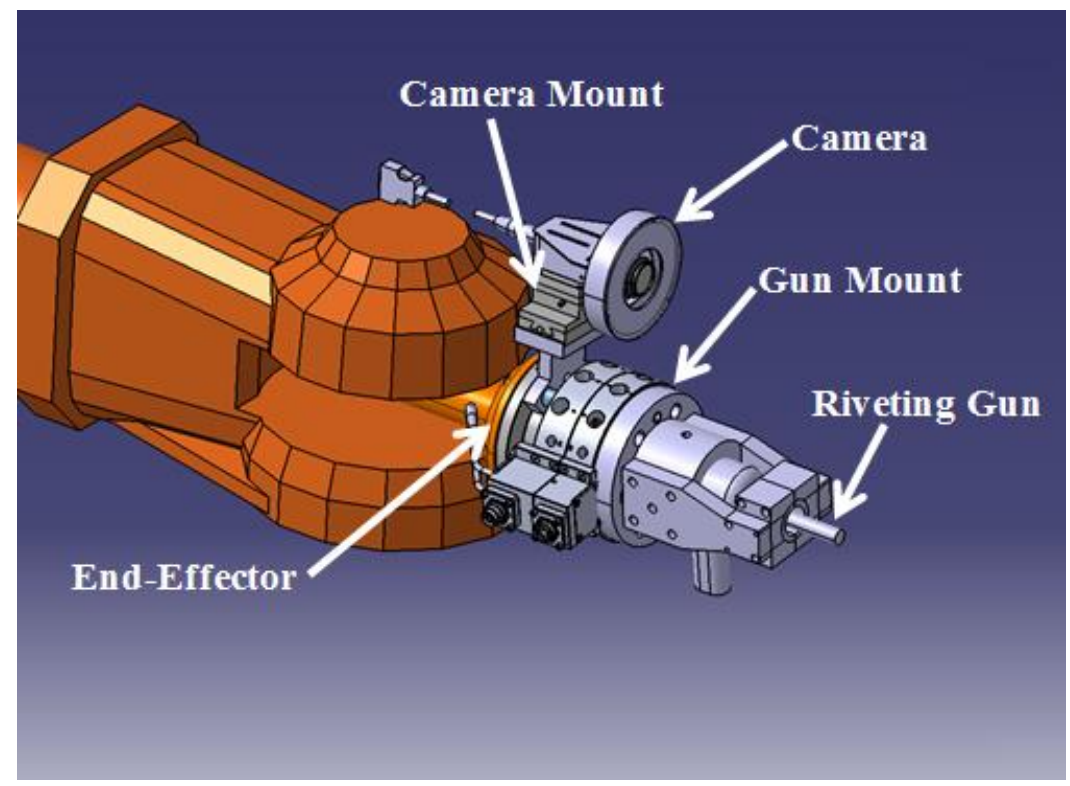

Figure 4-4: Robot Tool-Camera System CAD Model

The main idea of the new developed formulation is treating the tool as well as the camera as an extension of the kinematic model of the robotic arm. The theoretical model shown in Figure 4-5 was converted to the model shown in the diagram in Figure 4-6.

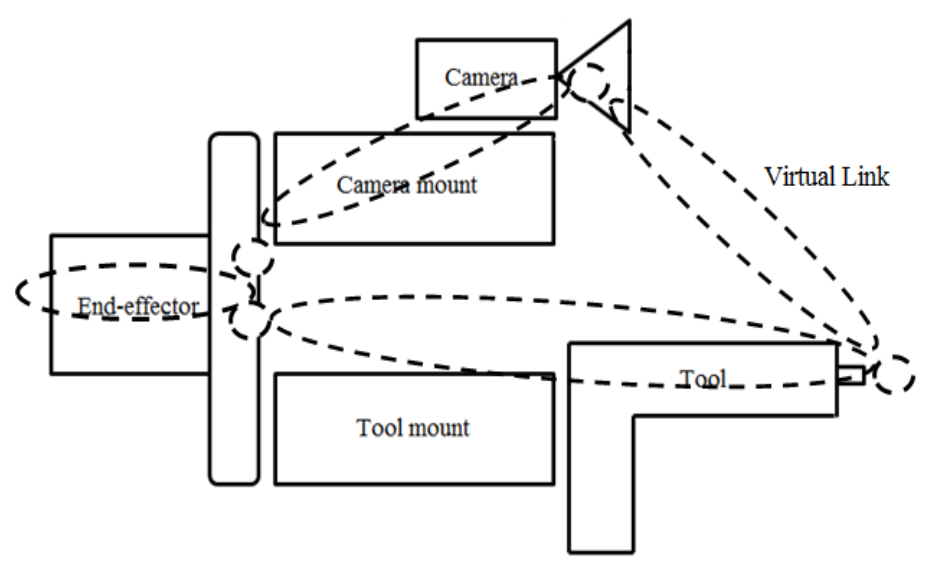

Figure 4-5: Superimposed Theoretical Robotic Links

The conversion from the model in Figure 4-5 to the model in Figure 4-6 is based on the transformation vector diagram of the system. It was concluded that the errors in the camera link will be eminently reflected through the tool link and the introduced virtual link. That led to the 
elimination of the camera link and achieving a two link extension of the robotic arm as shown in Figure 4-6.

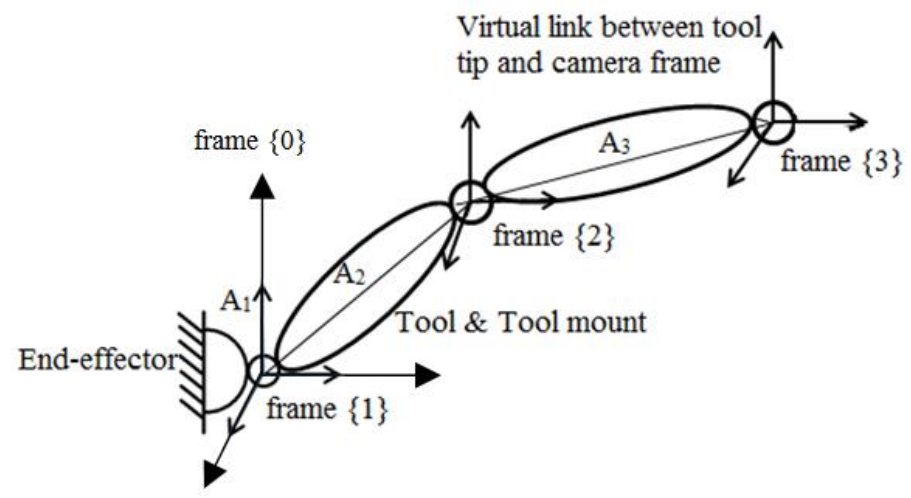

Figure 4-6: Final Theoretical model diagram.

The diagram shown in Figure 4-6 above is an extension of the robotic manipulator model. By considering that the manipulator was calibrated before the tooling calibration is performed, the errors at the tip are therefore due to the last two links. The self-calibration algorithm could then be applied to the robotic manipulator in addition to the tool and camera extension. The $\Delta \boldsymbol{g}$ in this case is a $12 \times 1$ vector since there are two links with six geometric errors for each.

The robotic tooling self-calibration algorithm is a technique where the measurement device (camera) is being held by the manipulator that requires calibration. This set-up is called eye-in-hand as discussed in the previous chapter. The errors due to the extension links are captured in the camera measurements of the holes distances which are compared against the actual holes distances.

An analysis of the limitations of the robotic tooling self-calibration could be verified and completely described by combining Equation 4.18 with Equation 4.41, 


$$
\begin{gathered}
G_{i j}=2\left(X_{C_{i}}-X_{C_{j}}\right)^{T}\left(R_{E_{i}}^{B} J_{E}-R_{E_{j}}^{B} J_{E}\right) \\
G_{i j}=2\left(X_{C_{i}}-X_{C_{j}}\right)^{T}\left(R_{E_{i}}^{B}-R_{E_{j}}^{B}\right) J_{E}
\end{gathered}
$$

For multiple measurements,

$$
G=\left[\begin{array}{c}
G_{i j, 1} \\
G_{i j, 2} \\
\vdots \\
G_{i j, n}
\end{array}\right]=\left[\begin{array}{c}
\left(X_{C_{i}}-X_{C_{j}}\right)_{1}^{T}\left(R_{E_{i}}^{B}-R_{E_{j}}^{B}\right)_{1} \\
\left(X_{C_{i}}-X_{C_{j}}\right)_{2}^{T}\left(R_{E_{i}}^{B}-R_{E_{j}}^{B}\right)_{2} \\
\vdots \\
\left(X_{C_{i}}-X_{C_{j}}\right)_{n}^{T}\left(R_{E_{i}}^{B}-R_{E_{j}}^{B}\right)_{n}
\end{array}\right] 2 J_{E}
$$

Since,

$$
\Delta \boldsymbol{g}=\boldsymbol{G}^{+} \Delta \boldsymbol{X}_{C_{\boldsymbol{A}}}
$$

Determining the pseudo inverse of $\boldsymbol{G}$ by combining the Equation 4.45 and Equation 4.42,

$$
\begin{aligned}
& \boldsymbol{G}^{\boldsymbol{T}} \boldsymbol{G}=2 \boldsymbol{J}_{\boldsymbol{E}}^{T}\left[( \boldsymbol { R } _ { \boldsymbol { E } _ { \boldsymbol { i } } } ^ { \boldsymbol { B } } - \boldsymbol { R } _ { \boldsymbol { E } _ { j } } ^ { \boldsymbol { B } } ) _ { 1 } ^ { T } ( \boldsymbol { X } _ { \boldsymbol { i } } - \boldsymbol { X } _ { \boldsymbol { j } } ) _ { 1 } ( \boldsymbol { R } _ { \boldsymbol { E } _ { \boldsymbol { i } } } ^ { \boldsymbol { B } } - \boldsymbol { R } _ { \boldsymbol { E } _ { \boldsymbol { j } } } ^ { \boldsymbol { B } } ) _ { 2 } ^ { T } \left(\boldsymbol{X}_{\boldsymbol{i}}\right.\right. \\
& \left.-\boldsymbol{X}_{\boldsymbol{j}}\right)_{2} \ldots\left(\boldsymbol{R}_{\boldsymbol{E}_{\boldsymbol{i}}}^{\boldsymbol{B}}-\boldsymbol{R}_{\boldsymbol{E}_{\boldsymbol{j}}}^{\boldsymbol{B}}\right)_{n}^{T}\left(\boldsymbol{X}_{\boldsymbol{i}}\right.
\end{aligned}
$$

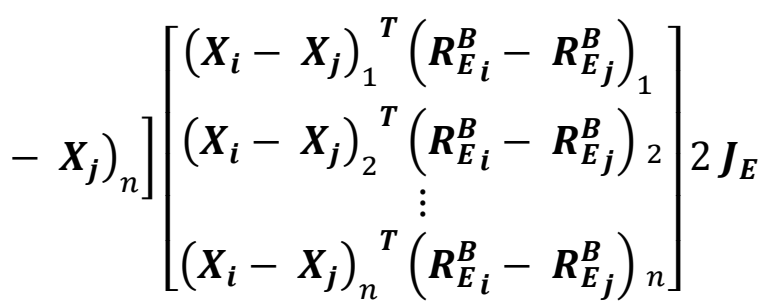




$$
\begin{aligned}
& \boldsymbol{G}^{\boldsymbol{T}} \boldsymbol{G}=2 \boldsymbol{J}_{\boldsymbol{E}}^{T}\left[( \boldsymbol { R } _ { \boldsymbol { E } _ { \boldsymbol { i } } } ^ { \boldsymbol { B } } - \boldsymbol { R } _ { \boldsymbol { E } _ { j } } ^ { \boldsymbol { B } } ) _ { 1 } ^ { T } ( \boldsymbol { X } _ { \boldsymbol { i } } - \boldsymbol { X } _ { \boldsymbol { j } } ) _ { 1 } ( \boldsymbol { R } _ { \boldsymbol { E } _ { \boldsymbol { i } } } ^ { \boldsymbol { B } } - \boldsymbol { R } _ { \boldsymbol { E } _ { j } } ^ { \boldsymbol { B } } ) _ { 2 } ^ { T } \left(\boldsymbol{X}_{\boldsymbol{i}}\right.\right. \\
& \left.-\boldsymbol{X}_{\boldsymbol{j}}\right)_{2} \ldots\left(\boldsymbol{R}_{\boldsymbol{E}_{\boldsymbol{i}}}^{\boldsymbol{B}}-\boldsymbol{R}_{\boldsymbol{E}_{\boldsymbol{j}}}^{\boldsymbol{B}}\right)_{n}^{T}\left(\boldsymbol{X}_{\boldsymbol{i}}\right. \\
& \left.\left.-\boldsymbol{X}_{j}\right)_{n}\right]\left[\begin{array}{c}
\left(\boldsymbol{X}_{\boldsymbol{i}}-\boldsymbol{X}_{\boldsymbol{j}}\right)_{1}{ }^{T}\left(\boldsymbol{R}_{E_{i}}^{\boldsymbol{B}}-\boldsymbol{R}_{E_{j}}^{\boldsymbol{B}}\right)_{1} \\
\left(\boldsymbol{X}_{\boldsymbol{i}}-\boldsymbol{X}_{\boldsymbol{j}}\right)_{2}{ }_{2}^{T}\left(\boldsymbol{R}_{E_{i}}^{\boldsymbol{B}}-\boldsymbol{R}_{\boldsymbol{E}_{j}}^{\boldsymbol{B}}\right)_{2} \\
\vdots \\
\left(\boldsymbol{X}_{\boldsymbol{i}}-\boldsymbol{X}_{\boldsymbol{j}}\right)_{n}{ }^{T}\left(\boldsymbol{R}_{E_{i}}^{\boldsymbol{B}}-\boldsymbol{R}_{\boldsymbol{E}_{j}}^{\boldsymbol{B}}\right)_{n}
\end{array}\right] 2 \boldsymbol{J}_{E}
\end{aligned}
$$

By simplifying the previous equation,

$$
\boldsymbol{G}^{\boldsymbol{T}} \boldsymbol{G}=4 \boldsymbol{J}_{\boldsymbol{E}}{ }^{T} \boldsymbol{D} \boldsymbol{J}_{\boldsymbol{E}}
$$

where $\boldsymbol{D}$ is a $3 \times 3$ matrix of the measurements as described in Equation 4.48 and $\boldsymbol{J}_{\boldsymbol{E}}$ is a $3 \times 12$ Jacobian matrix. Then the following condition must be true,

$$
\operatorname{rank}\left(\boldsymbol{J}_{\boldsymbol{E}}{ }^{T} \boldsymbol{D} \boldsymbol{J}_{\boldsymbol{E}}\right) \leq 3
$$

The root cause of the limitations of the self-calibration algorithm could be traced back to Equation 4.50. As illustrated using this equation, the rank of the $\boldsymbol{G}^{\boldsymbol{T}} \boldsymbol{G}$ which is the invertible portion of the pseudo inverse has a maximum rank of 3 while the size of the matrix is $12 \times 12$. Since the invertible portion is rank deficient (singular) given multiple measurements, the selfcalibration formulation is proven to be in adequate approach for the determination of the tool camera geometric errors. 


\subsubsection{Off-line Error Measurement for Non-linear Tool Calibration formulation}

The robotic tooling self-calibration algorithm is formulated such that the robotic manipulator has to be moved to different locations in the workspace. The movement is necessary for the determination of the distances between the holes on the mounted jig. Hence, the robot has to be operating/on-line during acquiring the measurements for performing the calibration process.

Contrary to the robotic tooling self-calibration algorithm, the nonlinear tool calibration formulation developed in section 4.3 has the advantage of being an off-line error measurement technique. The nonlinear tool calibration algorithm is formulated such that all the measurements could be acquired using the $3 \mathrm{D}$ vision system without moving the robotic manipulator. The measurements related to the end-effector position as well as the measurements related to the tool orientation are obtained while the robot is fully static/off-line.

The advantage of the off-line error measurement against the on-line error measurement is focused in the simplicity of its application. For the tool calibration, the required set-up is limited to the $3 \mathrm{D}$ vision system for digitizing the shapes required in addition to a computer. The data could be obtained by the user and fed into the calibration algorithm developed for determining the errors in each axis. The user can then correct the axes that require calibration based on the tool application. This process could be performed on the workshop floor for multiple robotic systems with a very short manufacturing down time compared to the current calibration processes that take a significant time and skill to be performed. 


\section{CHAPTER 5: MEASUREMENTS SIMULATIONS}

This chapter includes all the necessary simulations regarding every aspect of the measurement process. The simulations in this chapter are not only used to validate the developed approaches but also for determining the limitations and provide guidance for performing the final experiments. The first section of this chapter discusses the measurement system which will be used for obtaining the experimental data. The second and the third sections present the circle fitting and cylinder fitting methods which will be implemented for performing the tool calibration. The last two sections of this chapter provide the methodology for determining the minimum number of data points required during the experiment for achieving the required accuracy.

\subsection{Measurement System}

The measurement system used for the experiments is a $3 \mathrm{D}$ vision system which is capable of digitizing any point in the space within its field of vision. The system is 3D Creator FP7000 from Boulder Innovation Group. The system is capable of digitizing any point within 10 meters field of view with an RMS error of 100 microns. The 3D Creator behaves similar to a Coordinate Measurement Machine (CMM) but with the advantage of freedom of movement.

The measurement system is composed of two main parts. The first and the main part is the camera unit, shown in Figure 5-1 below, which includes three line CCD sensors. The second part accompanied with the system is the hand-held device, shown in Figure 5-2 below. The CCD line sensors receive infrared light from multiple LED's located on the hand-held device. 

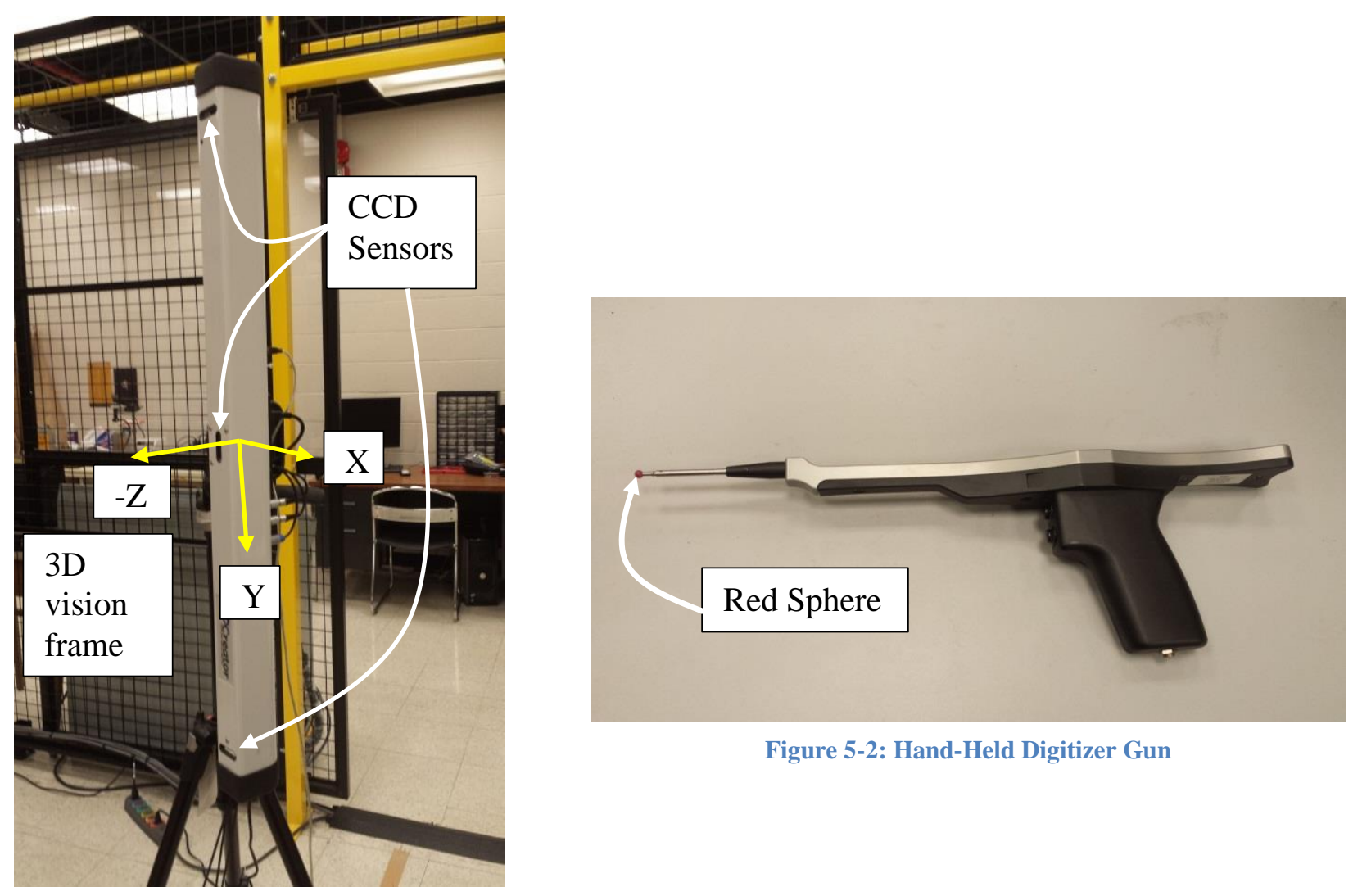

Figure 5-2: Hand-Held Digitizer Gun

Figure 5-1: Camera Unit

The measurement system operates by pointing the red sphere of the hand-held device to the required point. On pressing the button on the hand-held gun the point is digitized and captured by the camera unit. The camera unit uses a triangulation method based on the infrared LEDs and the three CCD sensors for the determination of the point. The point digitized is always located at the center of the red sphere of the hand-held gun and not at the surface of the sphere. The 3D vision system specifications are included in Appendix F. 


\subsection{End-Effector Position Estimation}

The first step of position calibration is the determination of the location of the center of the robot's end-effector. The robot's end-effector shown in Figure 5-3 below is a circular shaped disk that is designed for mounting the required tool. Using the 3D vision system to determine the center of the robot's end-effector, the following process was used.

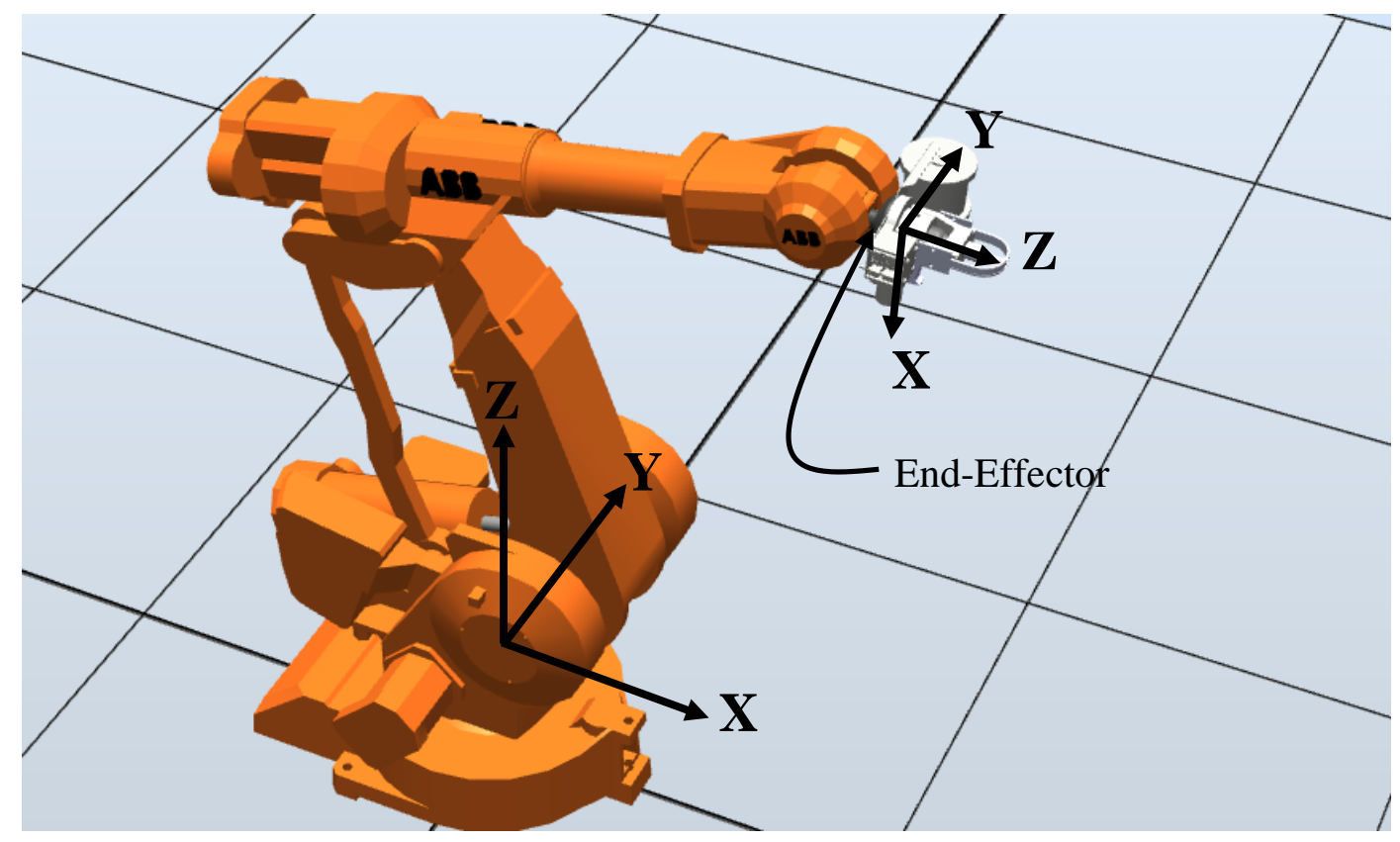

Figure 5-3: Robot's End-Effector

During the last few decades, there was a need for developing circle fitting methods and techniques for solving a range of problems in different fields. Physicists use circle fitting approaches for determining the energy of particles in an accelerator based on diameter of the circular path of the particles. Recently, in the field of robotics and mobile robotics, it was necessary to develop algorithms to detect circular objects using the aid of advanced circular laser systems. The determination of complex curves in computer vision is based on adding multiple circular arcs together which required developing circle fitting techniques [67]. 
Many circle fitting techniques were developed which could be mainly divided into two categories. The first category is the geometric circle fits while the second is the algebraic circle fits. Unlike the algebraic fits, all the geometric fits use an iterative procedure approach for improving the fitting accuracy. For complex applications, the normal approach followed for circle fitting is using an algebraic fit method to determine a good initial guess which is then followed by using an iterative geometric fit method to improve the accuracy. In general, the techniques used are mainly dependant on the application and the limitations of the problem.

Algebraic circle fitting techniques are usually simple, reliable and as accurate as geometric techniques if used under certain conditions. Two algebraic fit techniques were investigated for determining the center of the robot's end-effector with respect to the camera frame. The first technique is a method developed by Bullock [68] called "Least-Squares Circle Fit". The method is based on acquiring number of 2D points around the circumference of a circle in one frame followed by a transformation to different coordinates for solving a minimization problem. Finally, the solution has to be transformed back into the original coordinates.

The second technique is a simple algebraic fit technique called "Kasa method" [67]. Kasa method is considered to be the simplest algebraic method for circle fitting. Similar to the previous method, the Kasa method approach is to minimize the objective function given below,

$$
F=\sum f_{i}^{2}
$$

where

$$
f_{i}=\left(x_{i}-a\right)^{2}+\left(y_{i}-b\right)^{2}+r^{2}
$$


The following step is taking the derivative of the objective function with respect to each of $\mathrm{a}, \mathrm{b}$ and $\mathrm{r}$ parameters to minimize the errors between the acquired points and the circle parameters. The derivative of the objective function will lead to nonlinear equations which could be linearized using simple mathematical manipulation as explained in Appendix B.

The main advantage of the Kasa method in addition to its simplicity and reliability is that it is capable of determining the exact circle instantly if the measured points lie on the circle. There are some conditions that need to be taken into consideration for obtaining accurate results using the Kasa method:

1. The Kasa method was proven to be as accurate as any geometric method if the data points were taken along the circumference of a full circle. And it was determined to be significantly inaccurate if the data was taken along a small arc.

2. The data points acquired have to be located very close to the circle. In other words, the noise in the data points must be very small. The method was proven to provide an exact geometric results if the noise standard deviation is equal or less than 0.1 .

Both methods were tested by generating multiple simulations with different conditions. The first method was proven to be very sensitive to the location of the data points along the circle. The second method was proven to be very accurate and adequate for the experiment conditions. The results for both fitting techniques are shown in the following figures. 
Table 5-1: Circle Fitting Methods Results

\begin{tabular}{|c|c|c|c|}
\hline & X & Y & R \\
\hline Actual Values & 3 & 4 & 5 \\
\hline Least Square Method & 2.9438 & 4.397 & 4.988 \\
\hline Kasa Method & 2.9999 & 4.000 & 4.999 \\
\hline
\end{tabular}

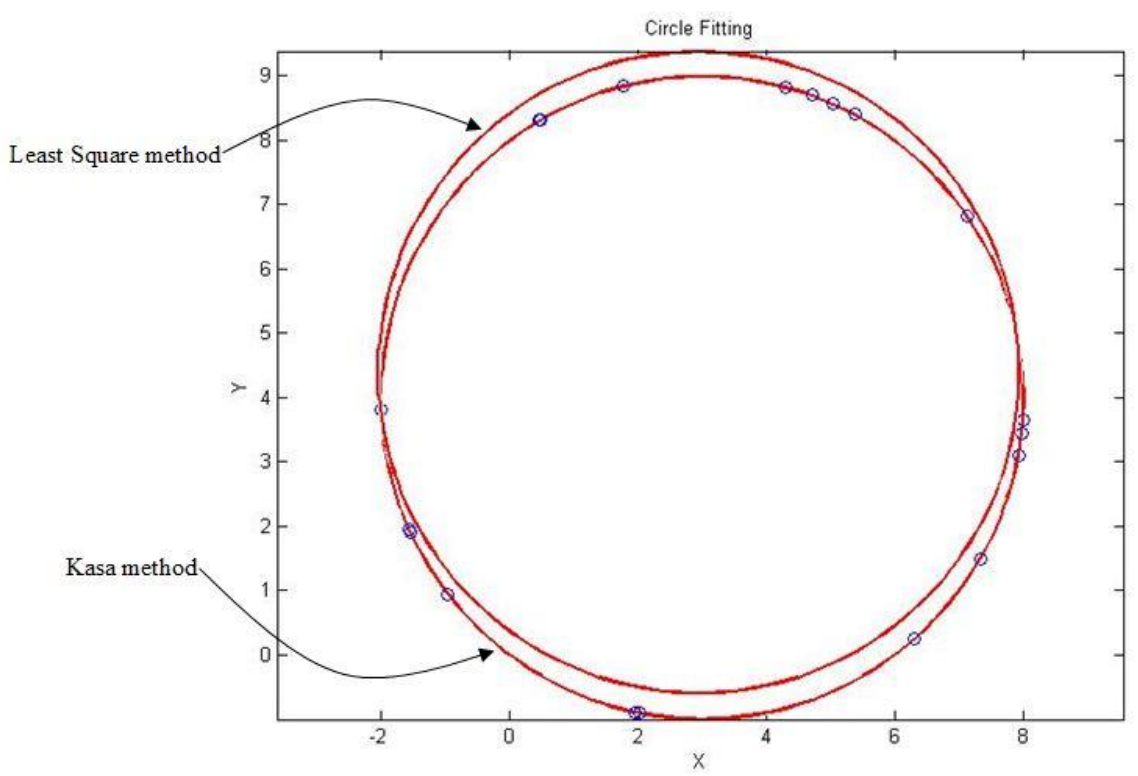

Figure 5-4: Circle Fitting using Kasa Method vs. Least Square Method

Both of the methods described above require 2D data points for determining the best fit circle parameters. However, the measurement system described in the previous section provides the $3 \mathrm{D}$ coordinates of any point within its field of vision. As a consequence, an algorithm has to be developed for fitting a circle in $3 \mathrm{D}$ space instead of $2 \mathrm{D}$ space.

An algorithm for fitting a circle in 3D space is shown in Figure 5-5. The algorithm starts by processing $3 \mathrm{D}$ data points obtained from the $3 \mathrm{D}$ vision system which resemble the shape of a circle in 3D space. The next step is generating a best fit plane by minimizing the perpendicular distances between all the points and the plane as explained in Appendix A. 


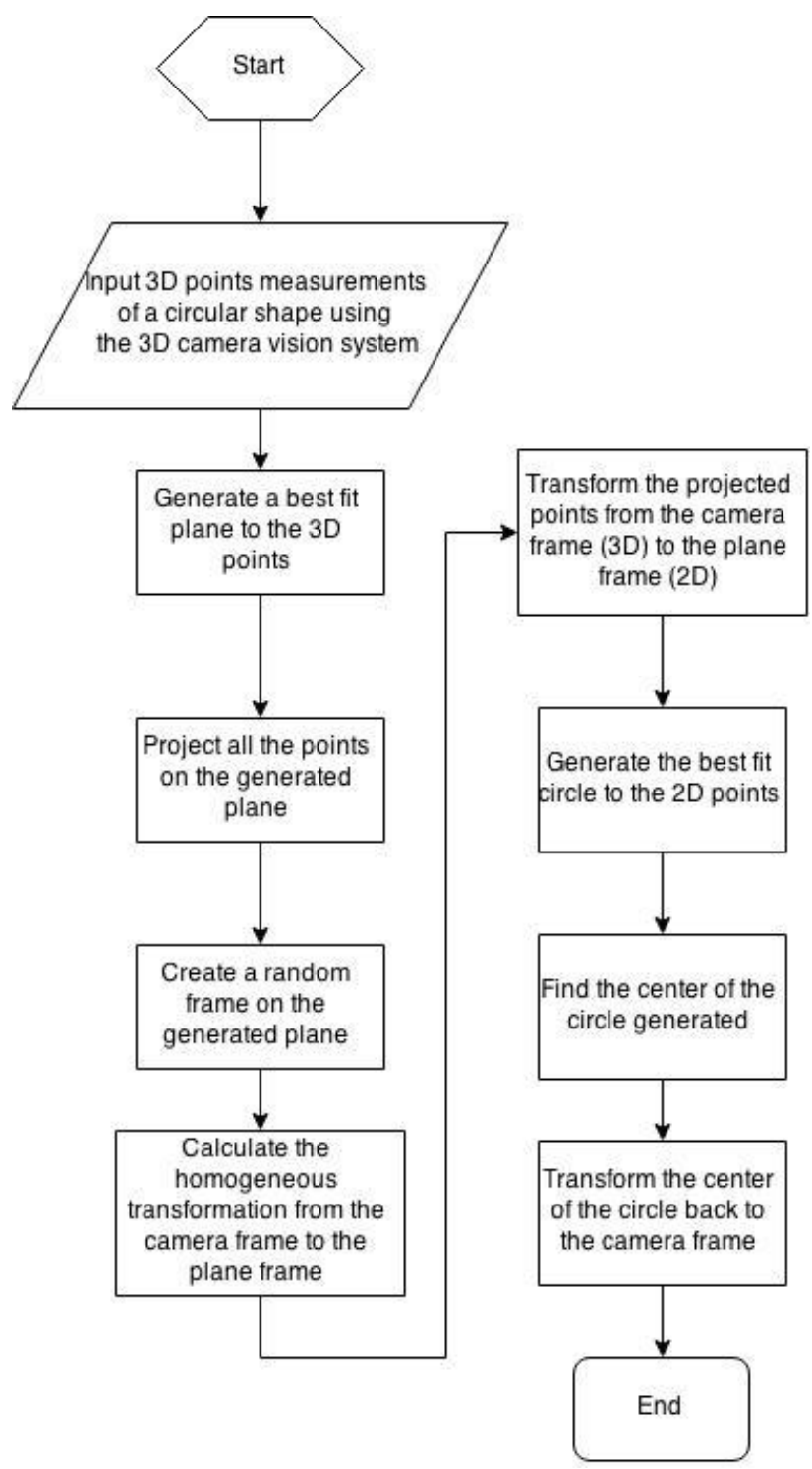

Figure 5-5: 3D Space Circle Fitting Algorithm

Afterwards, the 3D points are projected on the best fit plane and an arbitrary frame attached to the plane is created. The transformation from the camera frame to the plane frame is determined. Using the transformation, the projected points are transformed from the camera frame in which the points are $3 \mathrm{D}$ to the plane frame in which the points are $2 \mathrm{D}$. The circle fitting method (Kasa) is used for determining the best fit circle radius and center. Finally, the circle center is transformed back to the camera frame. 
The 3D circle fitting algorithm developed above along with Kasa method for 2D circle fitting were verified by simulation. As shown in Figure 5-6 below, circle data point generator was used to generate multiple data points generally oriented in $3 \mathrm{D}$ space. The data points are shown in Figure 5-6 as blue circular-shaped points. The points were used to generate the best fit plane which is then used for projecting the points on to it. The projected points are shown as the blue cross-shaped figures. The circle center was determined and transformed back to the 3D vision frame as represented by the large red cross-shaped figure.

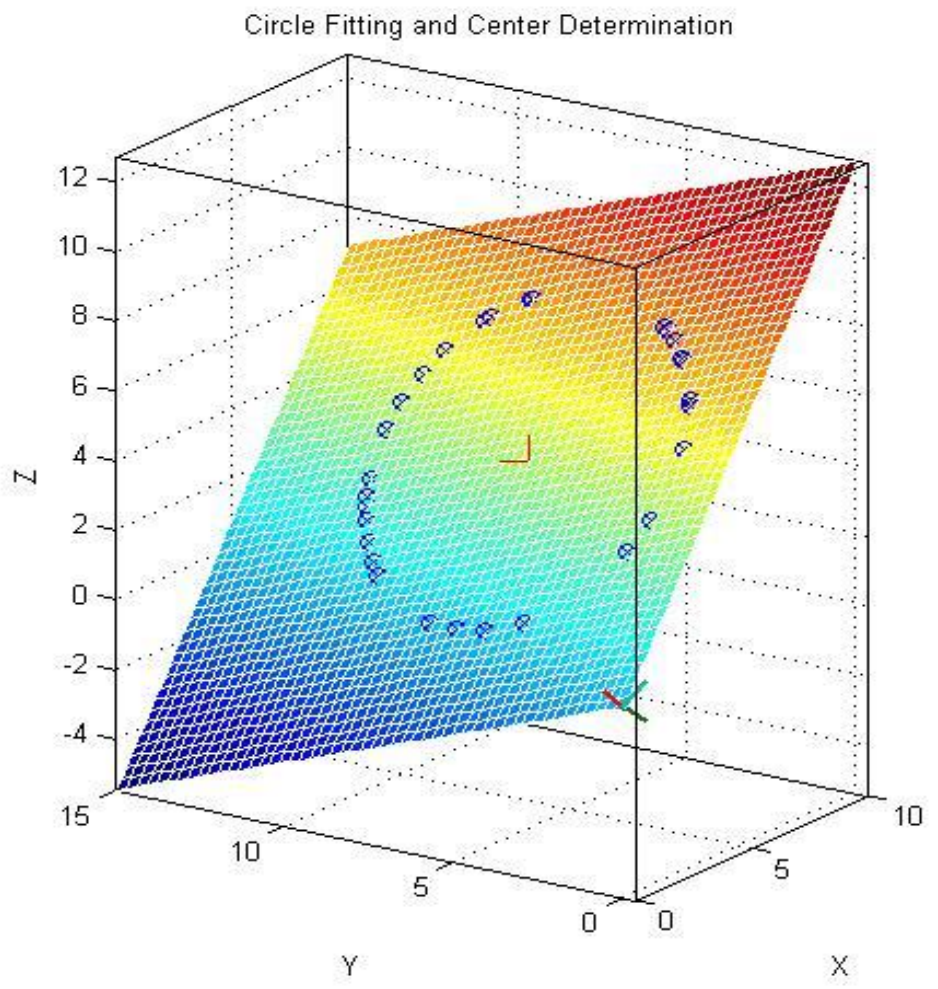

Figure 5-6: Simulated 3D Circle Fitting Algorithm

\subsection{Tool Pose Estimation}

For performing a full pose tooling calibration, the orientation of the tool must be known. The tool used in this robot set up as explained in the previous chapters is a pneumatic riveting 
gun as shown in Figure 5-7. The main problem is the determination of the orientation of the rivet gun. The first simple approach that could be used for determining the tool orientation is by obtaining only two points on the axis of the tool. The two points suggested were the end-effector center point and the tool center point (TCP). This approach was dismissed because of the following reasons:

1. The end-effector center point is definitely not on the tool axis due to the position errors of the tool mount assembly and manufacturing.

2. The tool center point (TCP) is very difficult to be determined accurately since the tool tip is movable and not rigid.

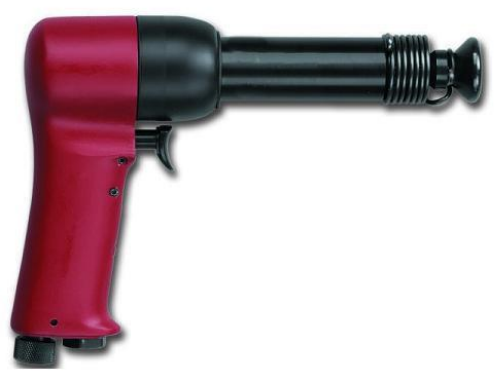

Figure 5-7: Pneumatic Rivet Gun

The second approach which is guaranteed to provide the tool orientation is fitting a cylinder to the cylindrical shaped rivet gun barrel. Determining the orientation axis of the fitted cylinder is the same as determining the orientation of the tool. The main idea is to use the 3D vision system to digitize multiple points on the surface of the rivet gun barrel. Afterwards, an optimization technique would be used to fit a cylinder shape to the obtained data points. The 
orientation of the cylinder axis with respect to the camera represents the orientation of the tool with respect to the camera.

Cylinder fitting algorithms were researched extensively for determining a simple cylinder fitting approach. Unfortunately, the problem of fitting a cylinder shape to 3D data points is considered to be a complicated problem due to the nonlinearity of the cylinder equations in general orientation in the space. A new method was formulated for cylinder fitting using genetic algorithm as a search method for determining the best fit cylinder parameters.

\subsubsection{Fitness Function}

The first step of using genetic algorithm for optimizing the cylinder parameters for obtaining the best fit cylinder is designing the fitness function. The fitness function is the cost function which will be minimized by the genetic algorithm by estimating the optimized parameters.

A generally oriented cylinder in the space could be completely described by seven parameters. Three parameters of which are used for describing the position of any point on the cylinder axis. Three more parameters are used for describing the orientation of the axis of the cylinder. The last parameter is used for describing the radius of the cylinder. The following assumptions were used for reducing the number of parameters to five,

1. The orientation of the cylinder axis will be described using the spherical coordinates. The spherical coordinate system uses only two angles which are the polar and azimuth angles. Using these two angles, the orientation of the cylinder axis could be easily determined. 
2. The axis of the cylinder is assumed to be in a general orientation in the space. Hence, the axis intersects the XY plane as shown in Figure 5-8. As a result, an easy point that lies on the axis of the cylinder is the intersection point with the $\mathrm{XY}$ plane where $\mathrm{Z}$ is equal to zero.

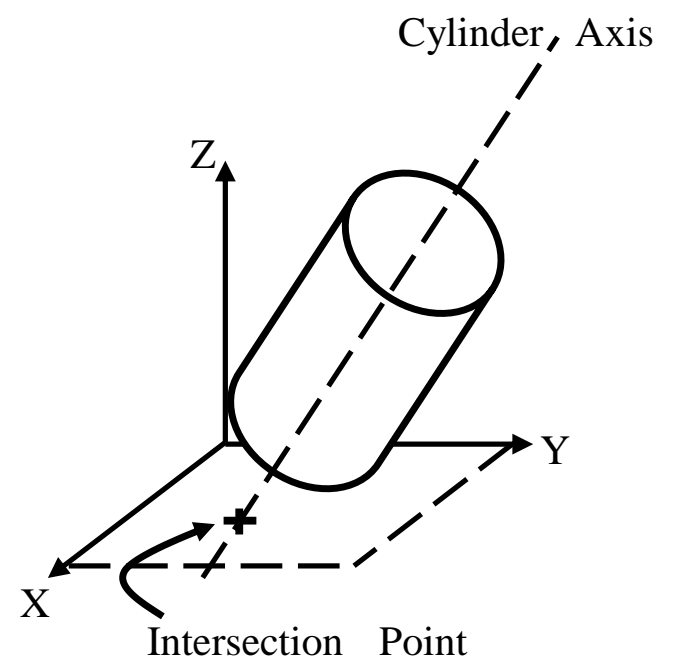

Figure 5-8: Cylinder in General Orientation

The equations that fully describe a cylinder which is generally oriented in space are given in Appendix C. The fitness function formulation is based on minimizing the errors between the radius of the cylinder and the distance from the axis to data points as described by the following equations,

$$
\begin{gathered}
\min (f(n)) \\
f(n)=\left\|{A_{i}}^{2}+{B_{i}}^{2}-r_{i}{ }^{2}\right\|_{2}
\end{gathered}
$$

where $r_{i}$ is the radius of the cylinder and $A_{i}$ and $B_{i}$ are given using the following equations,

$$
\begin{gathered}
A_{i}=\left(x_{i}-x_{c}\right) \operatorname{Cos}(\theta) \operatorname{Sin}(\varphi)+\left(y_{i}-y_{c}\right) \sin (\theta) \operatorname{Cos}(\varphi)-z_{i} \operatorname{Sin}(\varphi) \\
B_{i}=-\left(x_{i}-x_{c}\right) \operatorname{Sin}(\theta)-\left(y_{i}-y_{c}\right) \operatorname{Cos}(\theta)
\end{gathered}
$$


The developed cylinder fitting algorithm was verified by simulating 3D point cloud of a cylinder generally oriented in space as shown in Figure 5-9 below. The parameters of the generated data points are given in Table 5-2. The generated data points were used in the GA for estimating the best fit cylinder parameters. The best fitness value is $1.29647 \times 10^{-5}$, shown in Figure 5-10, given the GA was set to 500 generations and 500 population size. The GA cylinder estimated results are tabulated in Table 5-3 below.

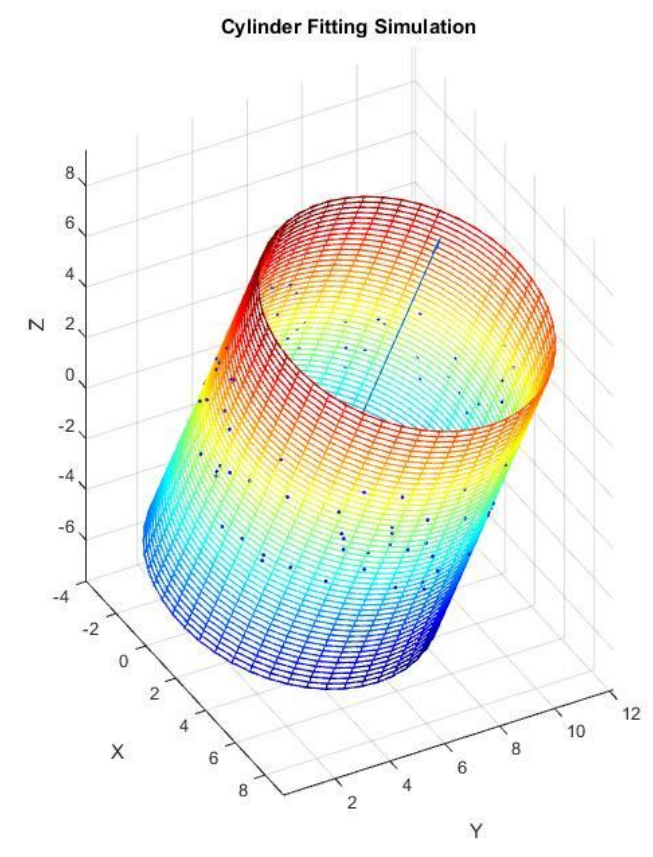

Figure 5-9: Generated Point Cloud and Best Fit Cylinder

Table 5-2: Simulated Cylinder Data Points Parameters

\begin{tabular}{|c|c|c|c|c|}
\hline $\mathbf{X}(\mathbf{m m})$ & $\mathbf{Y ( m m )}$ & Radius (mm) & $\boldsymbol{\theta}(\mathbf{d e g})$ & $\boldsymbol{\phi ( d e g )}$ \\
\hline 2.60802492 & 6.12454604 & 5 & 30 & 20 \\
\hline
\end{tabular}


Table 5-3: Estimated GA Cylinder Parameters

\begin{tabular}{|c|c|c|c|c|}
\hline $\mathbf{X}(\mathbf{m m})$ & $\mathbf{Y}(\mathbf{m m})$ & Radius (mm) & $\boldsymbol{\theta}(\mathbf{d e g})$ & $\boldsymbol{\phi}(\mathbf{d e g})$ \\
\hline 2.60802498 & 6.12454605 & 5 & 30.000012 & 19.9999937 \\
\hline
\end{tabular}

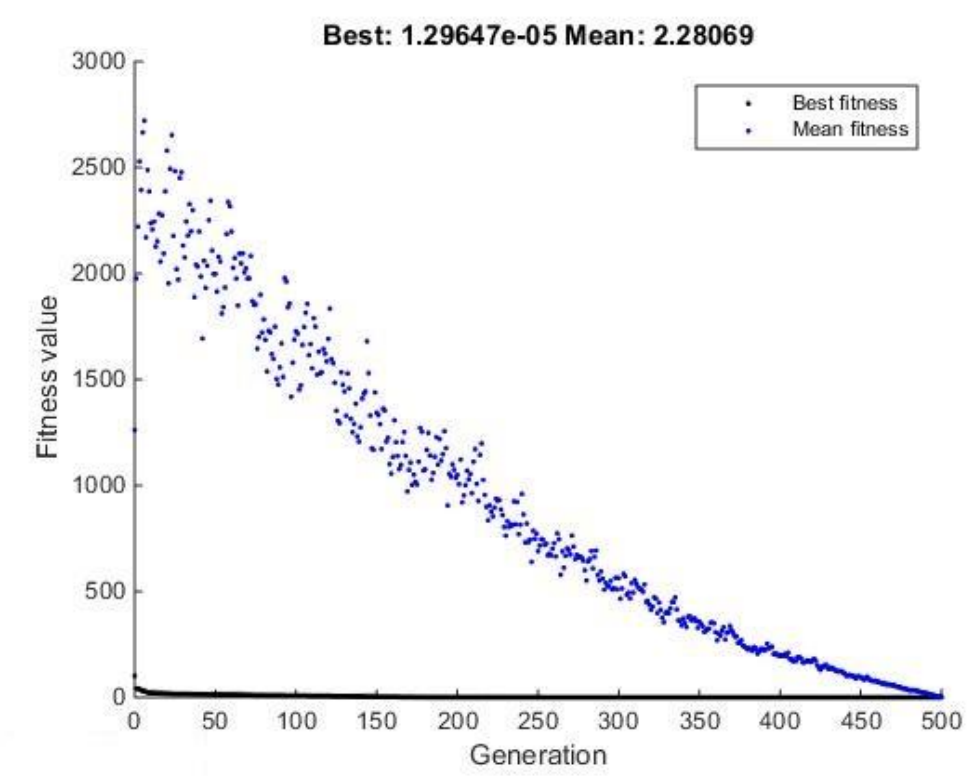

Figure 5-10: Cylinder Fitting GA with 500 Generations and 500 Population Size

\subsection{End-Effector Simulation}

A circular data point generator was created to simulate the measurement data points around the circumference of the end-effector disk. The simulation requires the number of data points needed by the user, the circle range which varies from 0 to $2 \pi$, the radius of the circle, the center of the circle before applying any transformation and finally the rotation matrix to transform the circle to an arbitrary position. 


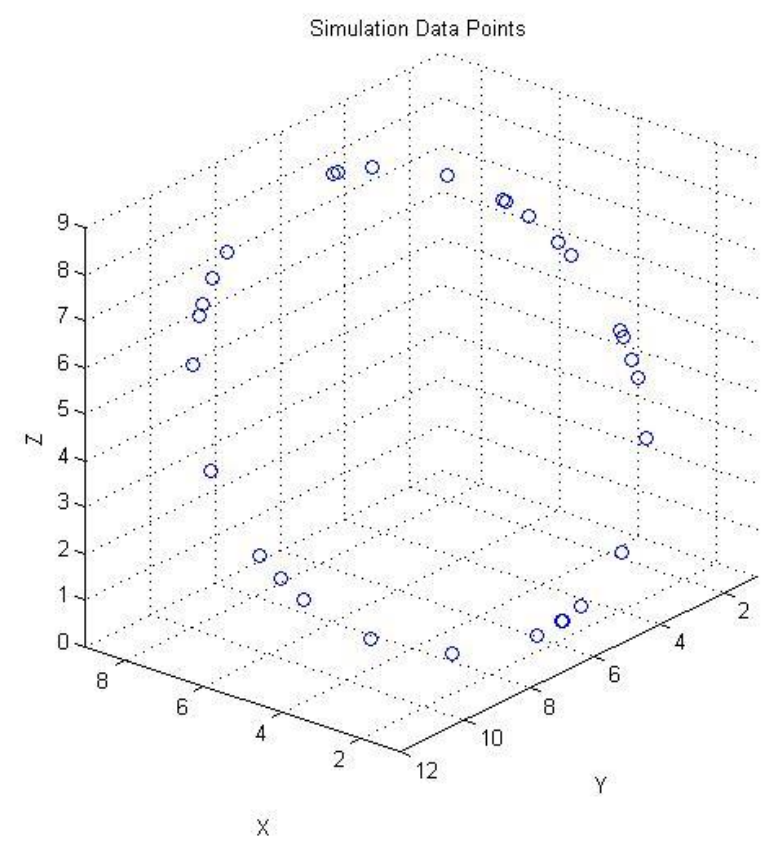

Figure 5-11: Circle Simualtion Data Points

The main purpose of this simulation is to test the effect of the number of data points, obtained randomly around the circumference of the end-effector, on the accuracy of the center location of the end-effector using the Kasa method for circle fitting. The generator was set to impose normally distributed noise with a variance of 0.1 to the data points. The variance was chosen to represent a system with less accuracy than the current used system. The graph in Figure 5-12 shows the effect of the number of points on the positional accuracy of the circle center location. The figure clearly shows that the errors drop from approximately 0.24 at 3 points to less than 0.05 at roughly 15 points.

It is concluded from this simulation that the minimum number of measurement points required for locating the center of the end-effector disk using the digitizer is 15 points. 


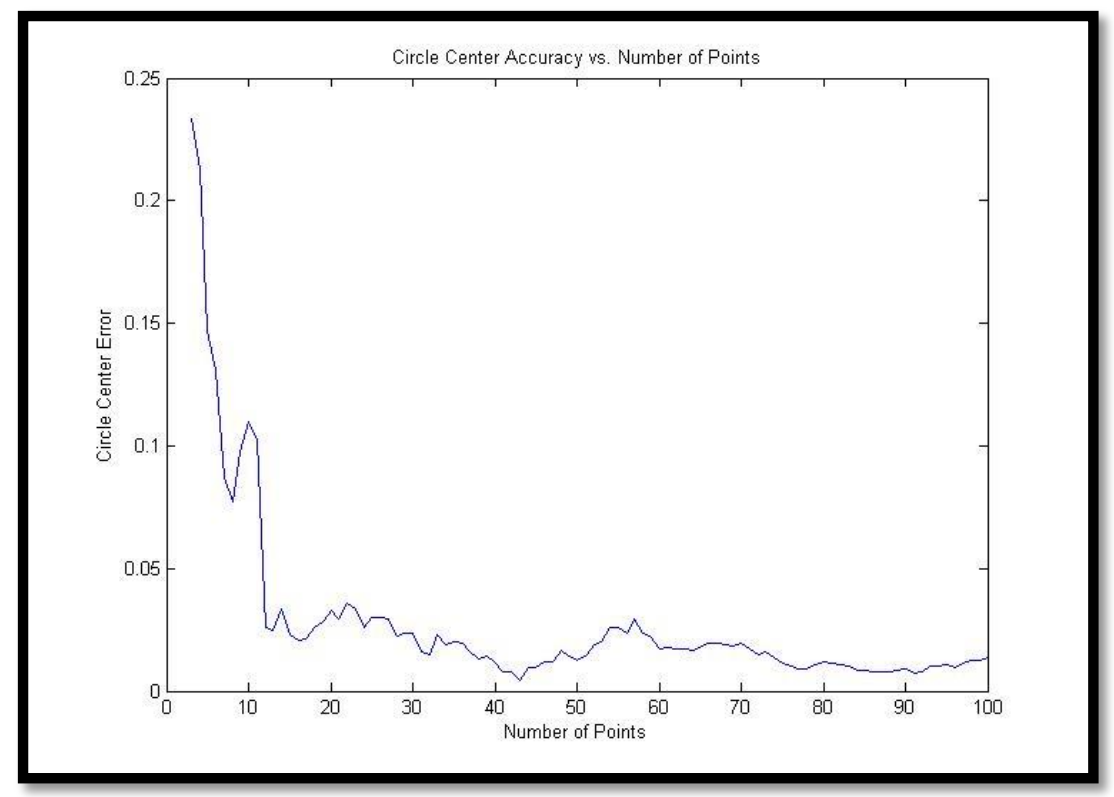

Figure 5-12: Circle Center Accuracy vs. Number of Points

\subsection{Tool Simulation}

A cylinder data point generator was created to simulate the $3 \mathrm{D}$ point cloud obtained by the $3 \mathrm{D}$ vision system. The generator could simulate a point cloud of a cylinder in a general orientation in the space. Multiple parameters could be specified using the generator such as the intersection of the axis of the cylinder with the $\mathrm{X}$ and $\mathrm{Y}$ plane before rotation. Other parameters include the radius of the cylinder, the range of the points generated around the cylinder's circumference as well as the range along the cylinder's axis. Also the number of points desired by the user and the rotation matrix for general placing the cylinder in a general orientation. A cylinder point cloud shown in Figure 5-13 was generated using 1000 points in a general orientation, a circumference range of $2 \pi$, an axis range from 0 to 5 units and a radius of 5 units. Normally distributed errors with variance of 0.1 were imposed on the data points to simulate the inaccuracies of the measurement system. 


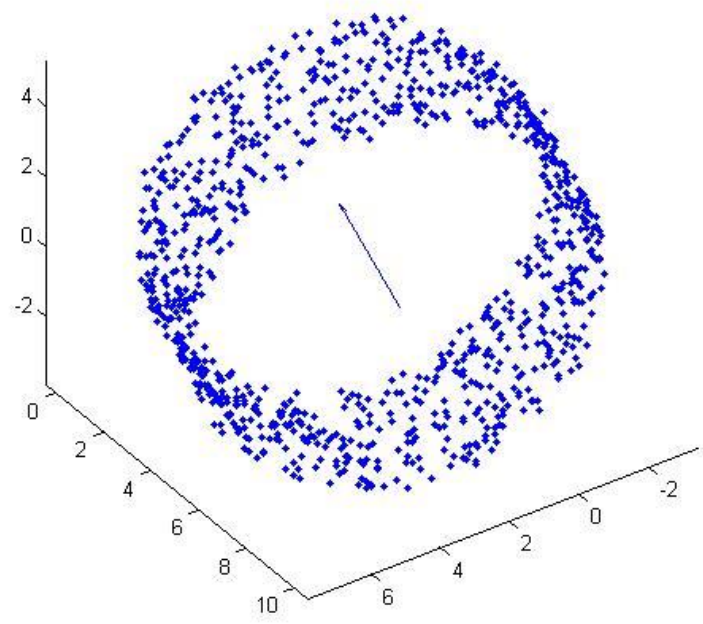

Figure 5-13: Cylinder Point Cloud with Noise

The 3D point was simulated using two different random generators. The first generator is used to simulate the points around the circumference of the cylinder as well as along its axis. This random generator must depict the operation of randomly registering points using the 3D vision system by a user. The generator used for this purpose uses a uniform distribution function. The second generator is used to create noise for each point of the data cloud. The noise must be simulated to replicate the accuracy of the $3 \mathrm{D}$ vision system. The generator used for creating the noise uses a normally distributed function. The difference between the two types of generators is shown in Figure 5-14 below.

The simulation was used to test the number of data points required for acquiring an accurate cylinder fit using genetic algorithm. The data point generator was used to create a point cloud for a randomly oriented cylinder. The following step was the execution of genetic algorithm multiple times for different numbers of data points. 


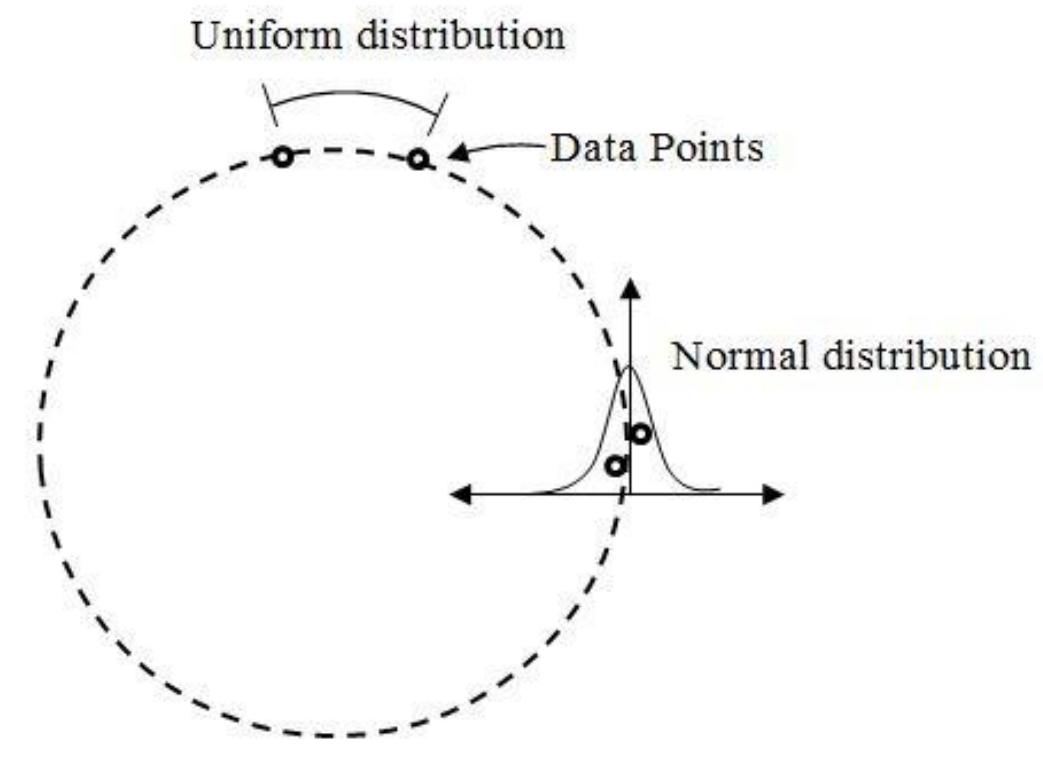

Figure 5-14: Normal Distribution and Uniform Distribution

The genetic algorithm was tested multiple times before determining the best set of parameters for the most accurate optimization results. The following results were obtained using the following set of genetic algorithm parameters which are a population size of 500 and 1000 generations and a stall limit of 1000 generations as well. Three main criteria were used for verifying the results of the fitting process which are, first the position errors, second is the orientation errors and finally radius errors. The position errors were determined by computing the Root Mean Square Position Error (RMSPE) of the point of intersection of the cylinder axis with the XY plane obtained from the fitting process and from the generated cylinder data points. The orientation errors were computed by determining the Root Mean Square Orientation Error (RMSOE) using the two spherical coordinates' angles $\theta$ and $\phi$. The radius error was computed for each set of number of points. 
The RMSPE versus the number of points determined from the simulation was plotted as shown in the Figure 5-15 below,

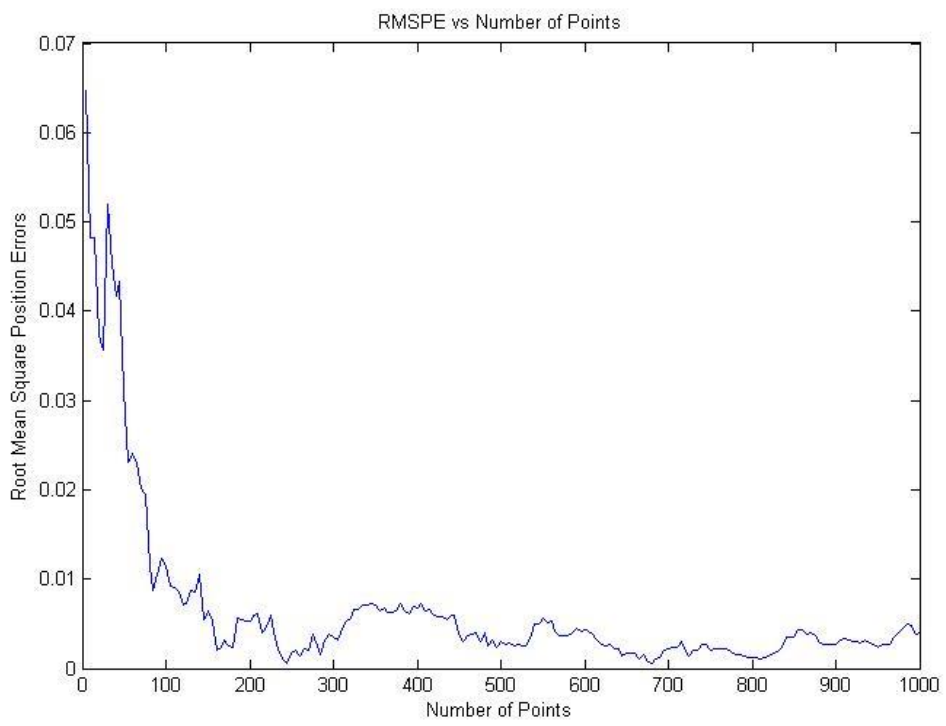

Figure 5-15: RMSPE vs. Number of Points

The RMSPE were noticed to be 0.07 by having only 5 data points. As shown in the figure, the RMSPE declines to less than 0.01 by increasing the number of data points to about 250 or 300 points and its average stays almost constant up to 1000 points.

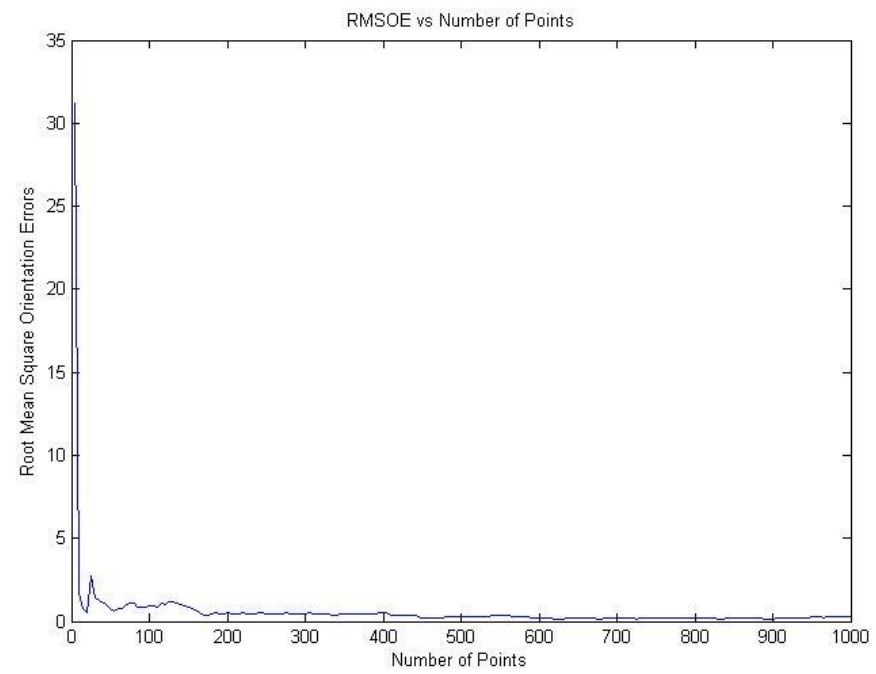

Figure 5-16: RMSOE vs. Number of Points 
The second criterion which is the RMSOE versus the number of points was plotted as shown in Figure 5-16.

The RMSOE was noticed to be above 30 with only 5 data points and it dropped sharply to less than 5 by increasing the data points to 20 . It gradually decreases to less than 1 by increasing the number of points to about 200.

Finally the radius error was plotted versus the number of points as shown in Figure 5-17 below. Similar to the RMSPE trend, the radius sharply reduced from 0.2 to less than 0.02 by increasing the number of data points from 5 to approximately 250 or 300 points.

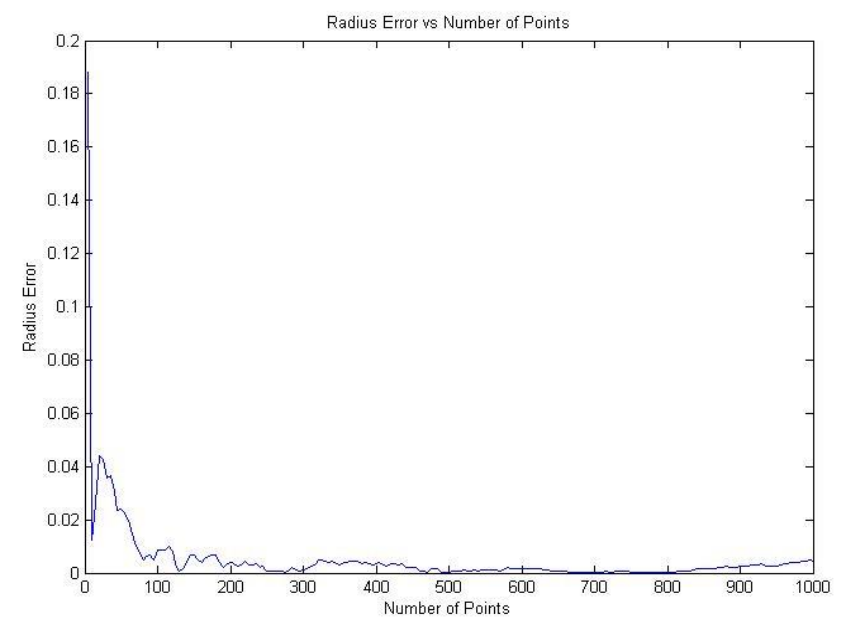

Figure 5-17: Radius Error vs. Number of Points

The simulation in this section was used to determine the minimum number of data points required by the user using the $3 \mathrm{D}$ vision system to obtain the optimum cylinder fit using genetic algorithm. A minimum number of 300 data points is required by the user to derive the errors to zero. 


\section{CHAPTER 6: EXPERIMENTS}

This chapter is dedicated to present and analyze the experimental application of the techniques developed in this thesis. The chapter is divided into five sections, two of which discuss the circle and cylinder fitting techniques while the other three discuss the position, orientation and full pose calibration respectively. The experiment was conducted based on the results and conclusions determined from the simulations presented in the previous chapter.

\subsection{End-Effector Pose Estimation}

The first step performed in the calibration process is locating the robot's end-effector position with respect to the camera frame. The process of determining the end-effector's frame starts by digitizing the circular end-effector plate. The camera's hand-held gun was used to determine multiple data points along the circumference of the end-effector. It was estimated based on the end-effector position estimation section that the minimum number of points required for fitting a circle to a number of points uniformly distributed on the circle is 15 points. For the experiment 58 points were used for ensuring better accuracy circle fitting, hence, better end-effector position estimation with respect to the camera frame. The data points were plotted and shown in Figure 6-1 below, 


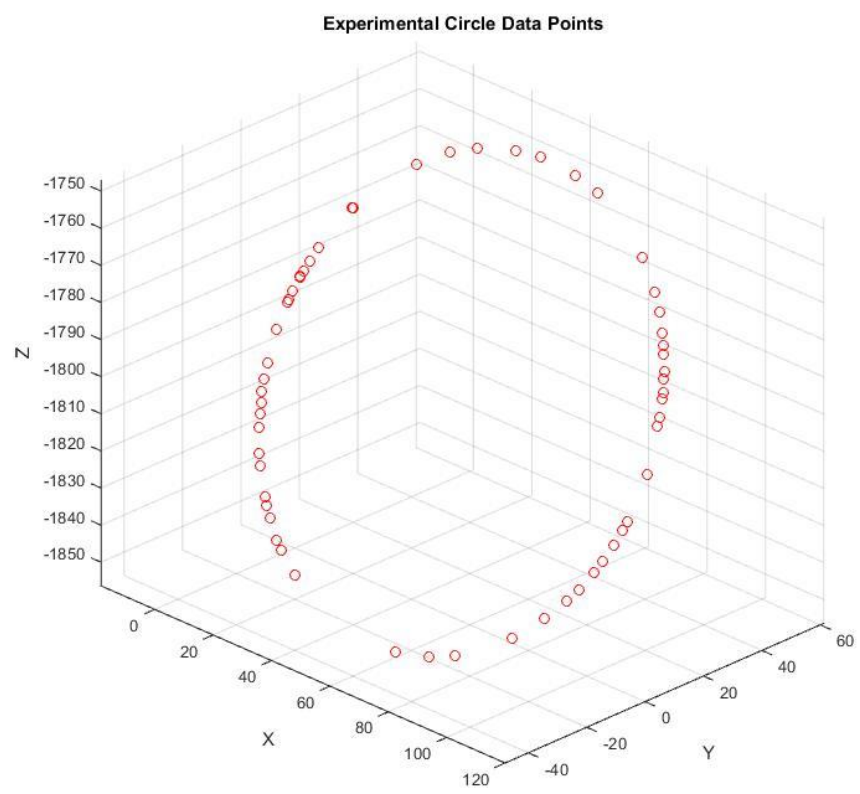

Figure 6-1: Experimental Circle Data Points (mm)

The circle fitting algorithm was applied on the experimental data points to determine the center of the circle. A plane was fitted to the data points; next the points were projected onto the plane. Finally, a circle that best fits the projected plane points was generated using Kasa method. The location of the circle center was then transformed back into the camera's frame. The location of the end-effector position with respect to the camera frame was tabulated in Table 6-1 below,

Table 6-1: End-Effector Location with Respect to the Camera Frame

\begin{tabular}{|c|c|c|}
\hline $\mathbf{X}(\mathbf{m m})$ & $\mathbf{Y}(\mathbf{m m})$ & $\mathbf{Z}(\mathbf{m m})$ \\
\hline $0.050797 \times 10^{3}$ & $0.006385 \times 10^{3}$ & $-1.80183 \times 10^{3}$ \\
\hline
\end{tabular}

According to the circle fitting algorithm described in the previous chapter, a frame has to be randomly constructed on the plane for transforming the data points from the camera frame to the plane frame. After the fitting process is complete, another frame has to be constructed which resembles the end-effector's frame. As explained in chapter 4, the robot's tool coordinates has its 
origin at the center of the end-effector plate. The $\mathrm{Z}$ axis is perpendicular to the end-effector's frame and the $\mathrm{X}$ axis is pointing downwards. Since only the center of the end-effector which is the origin of the frame was determined, an extra point is required for aligning the frame with the end-effector.

An alignment point located on the $\mathrm{X}$ axis of the end-effector's frame was obtained using the digitizer. Multiple measurements were taken for the point and an average was obtained. The $\mathrm{X}$ axis was then determined using the origin and the alignment point. Finally, the end-effector frame was constructed as shown in Figure 6-2 below,

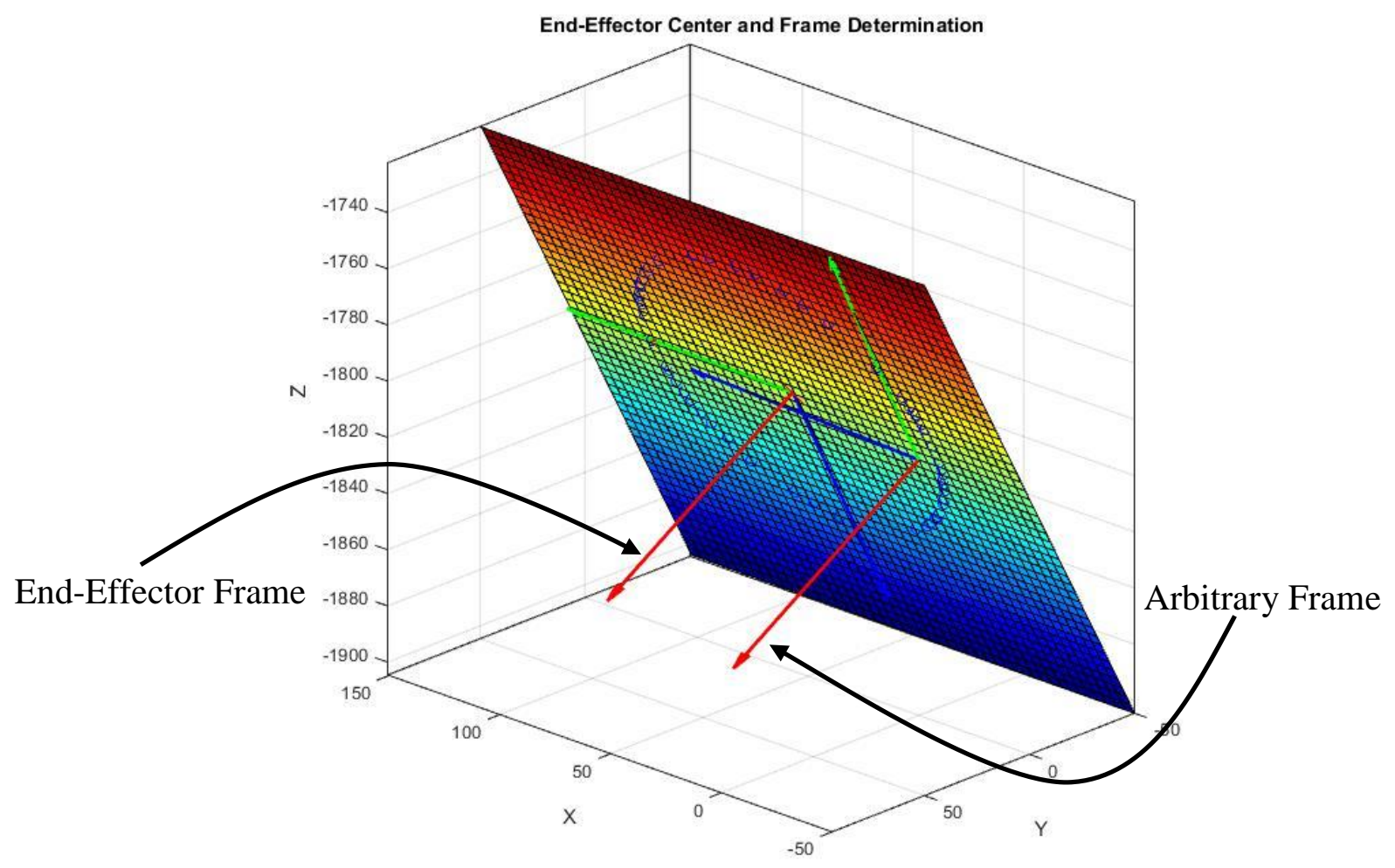

Figure 6-2: End-Effector Center and Frame Determination 


\subsection{Tool Pose Estimation}

The following step for performing the calibration process is the determination of the tool axis orientation and the tool center point location. The tool axis orientation is determined by obtaining a point cloud of the tool cylinder shape using the digitizer. It was concluded from the cylinder simulation that the minimum number of points required for obtaining the best cylinder fit is 300 points. The best cylinder fit was determined based on three criteria which are the position, orientation and the radius of the cylinder. For the experiment, a point cloud of 549 points, shown in Figure 6-3, were obtained for reducing the effect of noise on the optimization process.

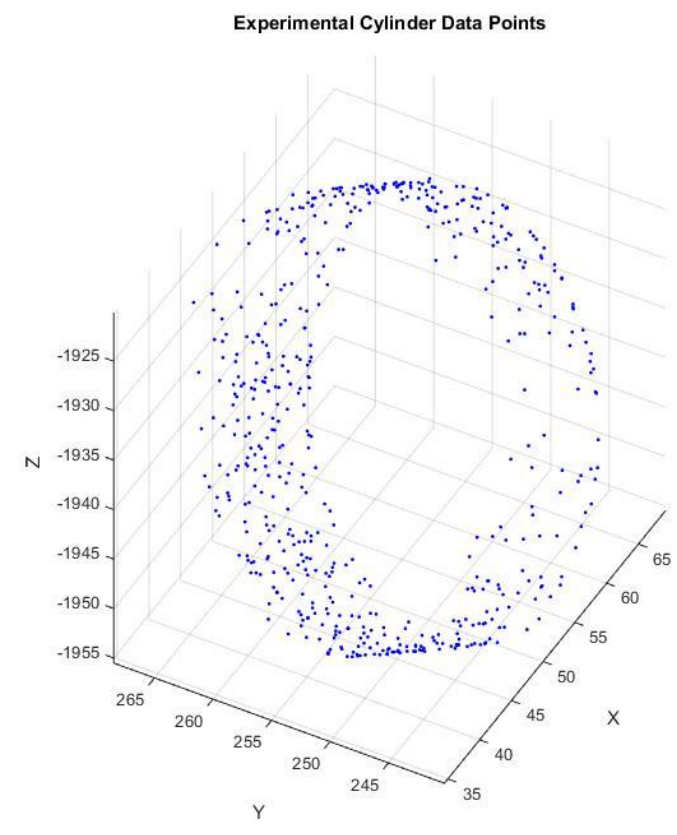

Figure 6-3: Experimental Cylinder Data Points (mm)

The next step is using genetic algorithm for optimizing the cylinder parameters for a best fit to the point cloud. The GA was set to 1000 generations and 500 population size. The results were obtained and compared to the actual cylinder parameters. The GA did not succeed in 
determining the best fit cylinder for the point cloud. The reason was found to be due to the position and orientation of the actual cylinder with respect to the camera frame. It was noticed that the position of the actual cylinder is very far from the camera frame compared to the simulation. It was also noticed that the cylinder axis orientation with respect to the camera's XY plane is close to being parallel. This is contrary to assumptions used for modelling the cylinder which states that the axis of the cylinder must not be parallel to the XY plane and that axis must intersect it. It was also verified using the simulation, that the GA does not return acceptable cylinder parameters values if the position of the cylinder is shifted to beyond $50 \mathrm{~mm}$ in any direction.

A proposed method for solving the given problem is to introduce an intermediate frame which is close to the data points and creates a better orientation angle with the cylinder axis. As shown in Figure 6-4, the Intermediate frame is a frame which is closer and better oriented with respect to the points. The points will be transformed to the intermediate frame where the cylinder fitting takes place. After the actual frame tool (rivet gun) frame is determined using the best fit cylinder parameters, the gun frame could be determined with respect to the camera frame using the following equation,

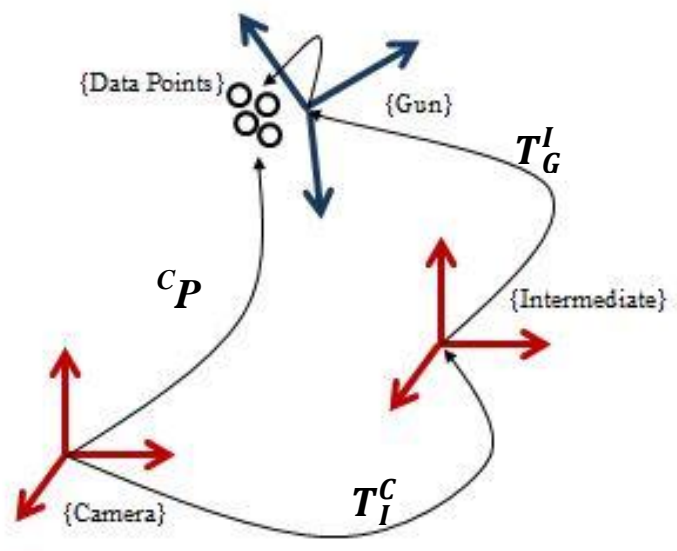

Figure 6-4: Introducing an Intermediate Frame Diagram 


$$
T_{G}^{C}=T_{I}^{C} T_{G}^{I}
$$

The intermediate frame position was determined by averaging all the cylinder data points and using the position of the averaged point as a position vector to the intermediate frame. The orientation was set to have the same orientation as the end-effector frame with respect to the camera frame. Genetic Algorithm was used again for fitting a cylinder to the data points with respect to the intermediate frame. For determining the best fit cylinder, the GA parameters were experimented with multiple times using 500 generations and different population sizes. The results could be found in Table 6-2 below,

Table 6-2: Cylinder GA Results with respect to the Intermediate Frame

\begin{tabular}{|c|c|c|c|c|c|}
\hline Population Size & $\mathbf{X}(\mathbf{m m})$ & $\mathbf{Y}(\mathbf{m m})$ & Radius $(\mathbf{m m})$ & $\boldsymbol{\theta}(\mathbf{d e g})$ & $\boldsymbol{\phi}(\mathbf{d e g})$ \\
\hline $\mathbf{1 0 0}$ & 2.9975 & 1.7335 & 17.4392 & 1.3776 & -0.05501 \\
\hline $\mathbf{2 0 0}$ & 2.9976 & 1.7335 & 17.4393 & 0.1932 & -0.04609 \\
\hline $\mathbf{3 0 0}$ & 2.9976 & 1.7334 & 17.4392 & 1.9190 & -0.06084 \\
\hline $\mathbf{4 0 0}$ & 2.9976 & 1.7335 & 17.4393 & 0.96771 & -0.05299 \\
\hline $\mathbf{5 0 0}$ & 2.9976 & 1.7336 & 17.4393 & -0.11928 & -0.04365 \\
\hline
\end{tabular}

The best fitness obtained is 131.784 using 500 Generations and 500 Population size shown in Figure 6-5. It was noticed that the fitness value does not change using more generations or bigger population sizes. 
By observing the results obtained in Table 6-2, it is clearly noticeable that the $\mathrm{X}$ and $\mathrm{Y}$ position as well as the Radius are stable and consistent given different population sizes. Unlike the $\mathrm{X}, \mathrm{Y}$ and Radius, the angles $\theta$ and $\varphi$ are fluctuating.

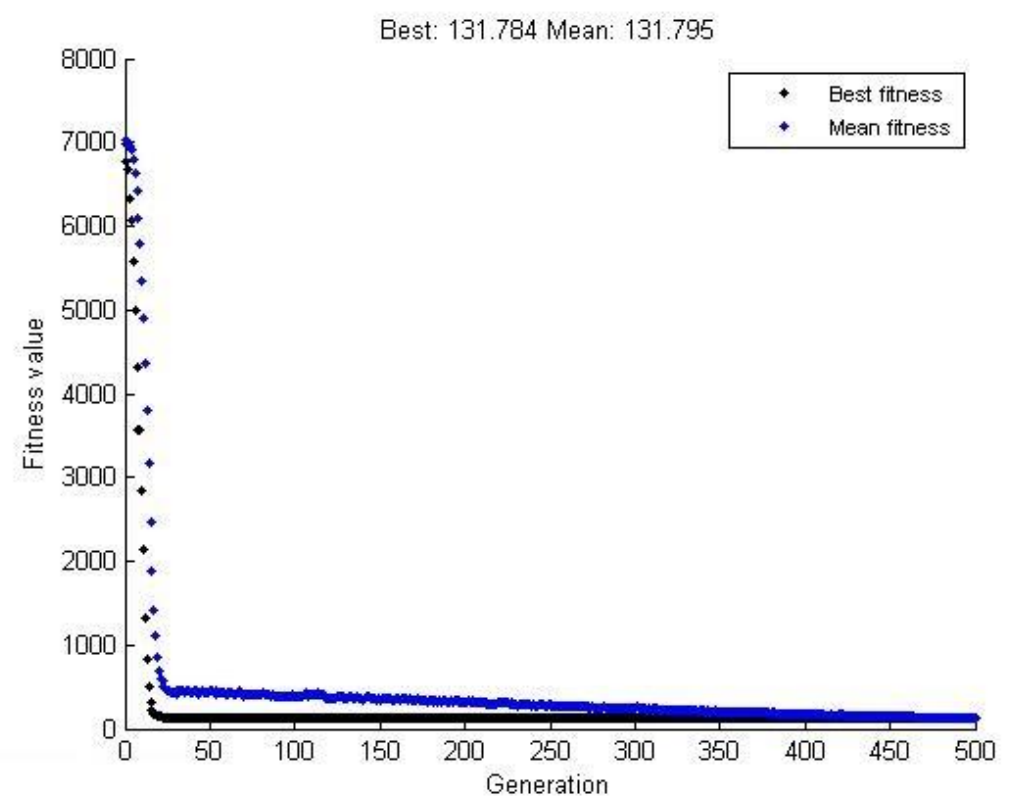

Figure 6-5: Cylinder Fitting GA with 500 Generations and 500 Population Size.

For acquiring a stable set of results for the angles, Monte Carlo estimate has to be applied for stabilizing the angles values. Monte Carlo estimate is a simple averaging method where the mean value of the estimated parameters is computed over multiple number of GA iterations as expressed in the following equation,

$$
\widetilde{g}(y)=\frac{1}{n} \sum_{i=1}^{n} g\left(y_{i}\right)
$$


Table 6-3: Monte Carlo Simulation Results

\begin{tabular}{|c|c|c|c|c|}
\hline $\mathbf{X}$ & $\mathbf{Y}$ & Radius (mm) & $\boldsymbol{\theta}(\mathbf{d e g})$ & $\boldsymbol{\phi}(\mathbf{d e g})$ \\
\hline 2.997638 & 1.733515 & 17.439245 & 1.314285 & -0.017301 \\
\hline
\end{tabular}

As shown in Figure 6-6, the obtained values were used to plot the cylinder and the experimental data points with respect to the intermediate frame.

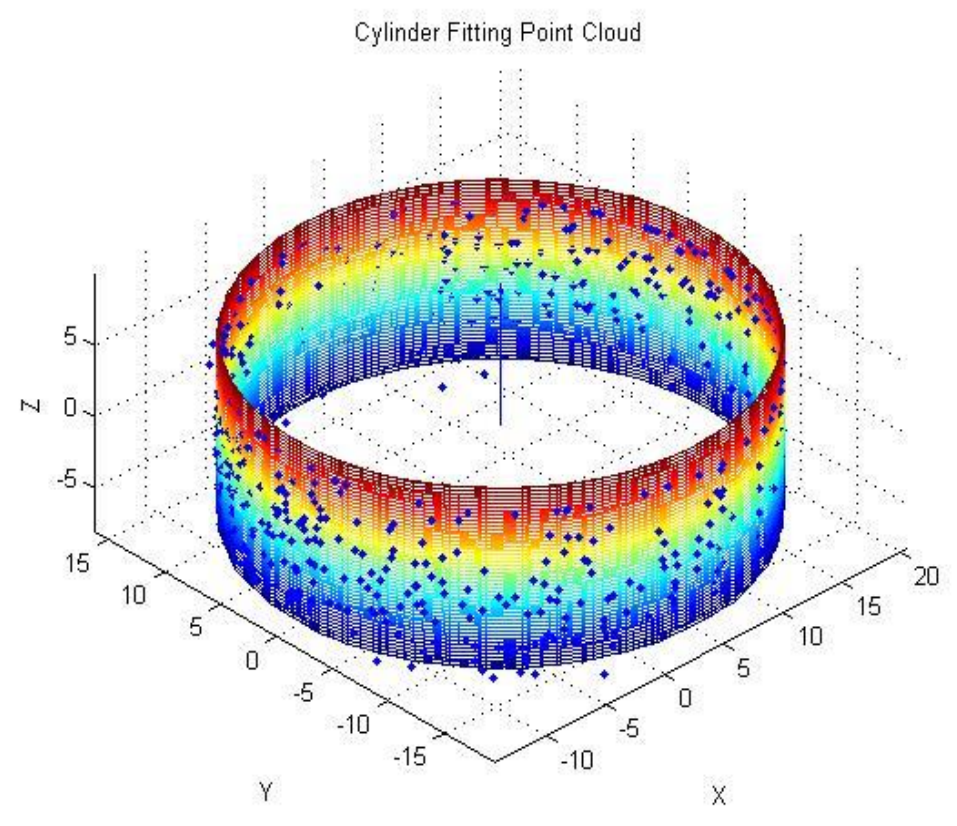

Figure 6-6: Cylinder Fitting Experimental Point Cloud

The cylinder parameters were used to determine the orientation of the tool (rivet gun) frame with respect to the end-effector. As shown in Figure 6-7, the diagram explains the camera, end-effector, gun set up with respect to each other. The orientation of the gun frame was determined with respect to the intermediate frame. The orientation of the end-effector frame was also determined with respect to the camera frame in the previous section. Using the following set 
of equations, the gun frame orientation could be easily determined with respect to the endeffector frame.

$$
\begin{aligned}
& R_{I}^{V} R_{G}^{I}=R_{E}^{V} R_{G}^{E} \\
& R_{G}^{E}=R_{E}^{V^{T}} R_{I}^{V} R_{G}^{I}
\end{aligned}
$$

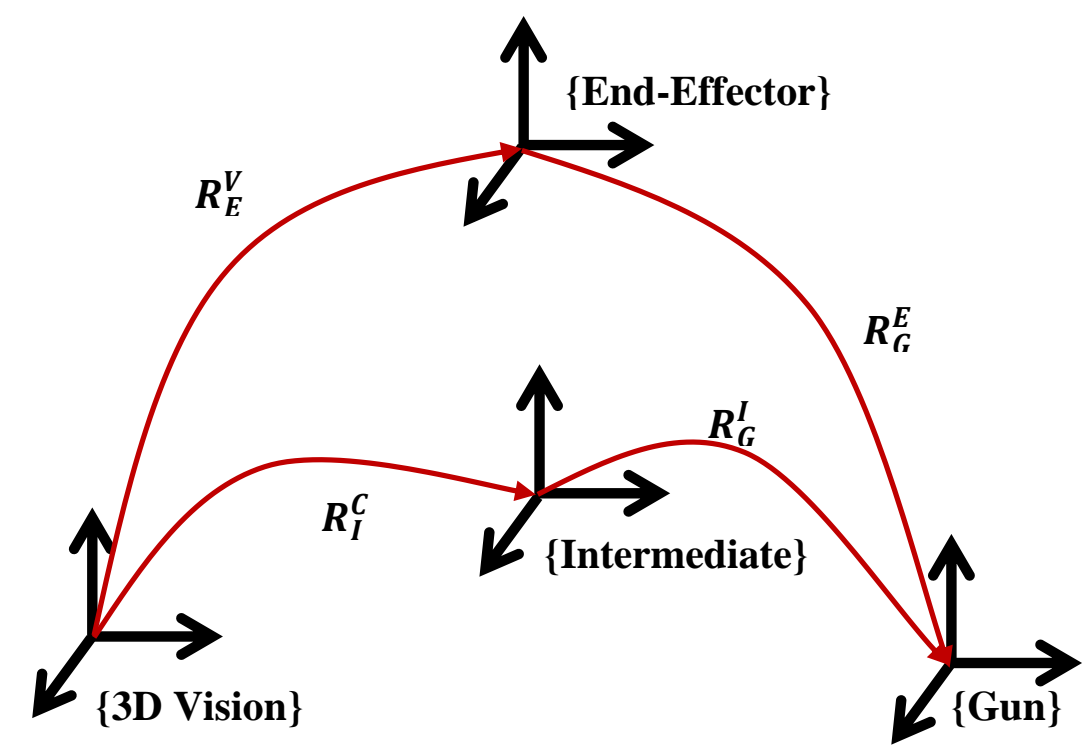

Figure 6-7: Camera, End-Effector and Gun Set-up Diagram

As described previously, the nominal orientation of the rivet gun frame perfectly matches the orientation of the end-effector frame based on the design CAD models. The orientation and position of both the measured frame and the nominal frame of the rivet gun with respect to the end-effector was compared and presented in Table 6-4.

Table 6-4: Measured and Nominal Rotation Matrices and Position Vectors

\begin{tabular}{|l|c|c|c|c|}
\hline & \multicolumn{3}{|c|}{ Rotation Matrix } & Position Vector (mm) \\
\hline \multirow{2}{*}{ Measured } & 0.99973901 & 0.02287969 & 0.00313807 & 50.71440250 \\
\cline { 2 - 5 } & 0.01369228 & -0.49987496 & 0.86606358 & 331.20808750 \\
\hline
\end{tabular}




\begin{tabular}{|l|c|c|c|c|}
\hline & 0.01864527 & -0.86578654 & -0.49992601 & -1980.231250 \\
\hline \multirow{3}{*}{ Nominal } & 0.99963924 & 0.02683525 & 0.00171706 & 51.433657 \\
\cline { 2 - 5 } & 0.01393145 & -0.48241207 & 0.87586855 & 330.970868 \\
\cline { 2 - 5 } & 0.02296312 & -0.87552865 & -0.48254672 & -1980.661388 \\
\hline
\end{tabular}

\subsection{Linear Full Pose Calibration}

By determining the tip pose errors using the nominal and the measured transformation of the tool frame with respect to the end-effector frame, the linear formulation for full pose calibration can be applied. As shown in Figure 6-8, the root mean square error (RMSE) converges from the value of approximately 3 to $1 \times 10^{-4}$ in only 4 iterations. The tool position and orientation geometric error is presented in Table 6-5. The calibrated rotation matrix and the position vector is also presented in Table 6-6 below.

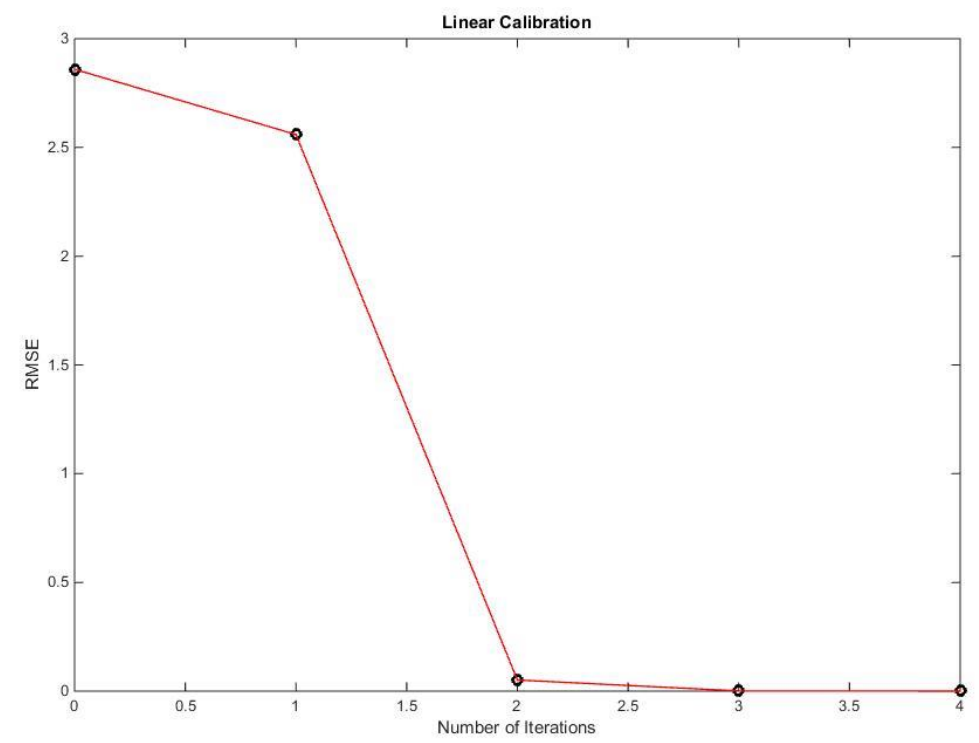

Figure 6-8: Linear Calibration RMSE vs. Number of Iterations 
Table 6-5: Linear Formulation Estimated Geometric Errors

\begin{tabular}{|c|c|c|c|c|c|}
\hline$\Delta \mathbf{x}(\mathbf{m m})$ & $\Delta \mathbf{y}(\mathbf{m m})$ & $\Delta \mathbf{z}(\mathbf{m m})$ & $\boldsymbol{\delta x}(\mathbf{d e g})$ & $\boldsymbol{\delta y}(\mathbf{d e g})$ & $\boldsymbol{\delta z}(\mathbf{d e g})$ \\
\hline-0.705815564 & -0.510370452 & 0.000462561 & -1.144895889 & 0.055203477 & 0.223368453 \\
\hline
\end{tabular}

Table 6-6: Calibrated Tool Pose Rotation Matrix and Position Vector

\begin{tabular}{|c|c|c|c|c|}
\hline & \multicolumn{3}{|c|}{ Rotation Matrix } & Position Vector (mm) \\
\hline \multirow{3}{*}{ Calibrated } & 0.998908940 & 0.046707348 & -0.001020997 & 51.040929326 \\
\cline { 2 - 5 } & 0.025909742 & -0.481775206 & 0.875946652 & 331.237715206 \\
\cline { 2 - 5 } & 0.039052347 & -0.875044604 & -0.482390830 & -1980.175216802 \\
\hline
\end{tabular}

\subsection{Nonlinear Position Calibration}

Using the measured and the nominal rivet gun frames results determined in the last section, the first nonlinear calibration process that will be applied is the position only calibration. The aim of this calibration is to correct the errors related to the position by excluding the orientation parameters from the fitness function.

The GA parameters were set to have 500 Generations and 100 Population size. The best fitness value determined under these conditions as shown in Figure 6-9 is $1.54239 \times 10^{-7}$. The position errors determined from the calibration process are tabulated in Table 6-7.

Table 6-7: Position Errors

\begin{tabular}{|c|c|c|}
\hline $\boldsymbol{\Delta \mathbf { X } ( \mathbf { m m } )}$ & $\boldsymbol{\Delta Y}(\mathbf{m m})$ & $\boldsymbol{\Delta Z}(\mathbf{m m})$ \\
\hline-0.705815507 & -0.51034175499 & 0.00097941593 \\
\hline
\end{tabular}


The position error was added to the nominal position vector to obtain the calibrated position vector presented in Table 6-8 below.

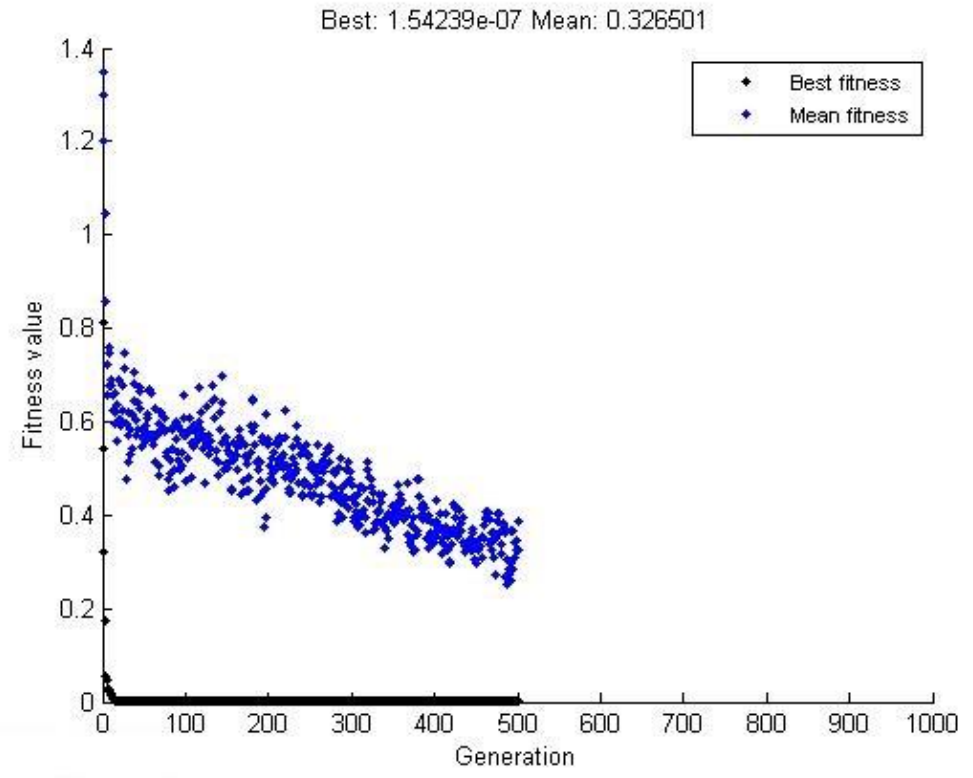

Figure 6-9: Position Only Calibration with 500 Generations and 100 Population Size

Table 6-8: Calibrated Position

\begin{tabular}{|c|c|c|}
\hline $\mathbf{X}(\mathbf{m m})$ & $\mathbf{Y ~ ( m m )}$ & $\mathbf{Z}(\mathbf{m m})$ \\
\hline 50.714403336 & 331.2080880877 & -1980.231250049 \\
\hline
\end{tabular}

\subsection{Nonlinear Orientation Calibration}

The next calibration process if the orientation only calibration which will be performed based on the nominal and measured rotation matrices of the rivet gun frame with respect to the camera's frame. By setting the weight factor of the position to zero and the weight factor of the orientation to one, the algorithm excludes the position parameters from the fitness function. 
The genetic algorithm parameters were set to multiple values for determining the best performance. The first trial was to assign the GA to run for 500 Generations and use a population size of 100 . The fitness value determined based on these conditions is 0.00141989 as shown in Figure 6-10 below.

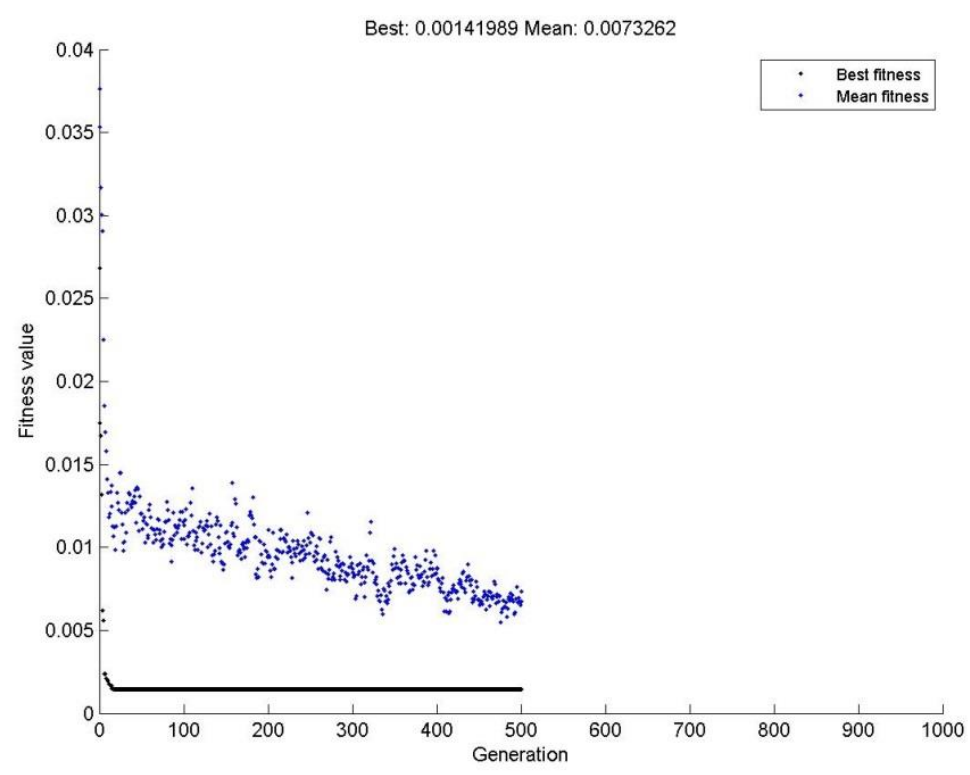

Figure 6-10: Orientation Only Calibration with 500 Generations and 100 Population Size

The orientation errors $\delta \mathrm{x}, \delta \mathrm{y}$ and $\delta \mathrm{z}$ values obtained from this calibration process are presented in Table 6-9 below,

Table 6-9: Orientation Errors for 500 Generations and 100 Population Size

\begin{tabular}{|c|c|c|}
\hline $\boldsymbol{\delta} \mathbf{x}(\mathbf{d e g})$ & $\boldsymbol{\delta y}(\mathbf{d e g})$ & $\boldsymbol{\delta z}(\mathbf{d e g})$ \\
\hline-1.14584428 & -0.02608992 & 0.22273761 \\
\hline
\end{tabular}


Using the orientation errors in the Table above, the calibrated rotation matrix was calculated and tabulated in Table 6-10 below along with the measured and the nominal rotation matrices for comparison.

Table 6-10: Measured, Nominal and Calibrated Rotation Matrices with respect to the Camera frame

\begin{tabular}{|c|c|c|c|}
\hline & \multicolumn{3}{|c|}{ Rotation Matrix } \\
\hline \multirow{3}{*}{ Measured } & 0.99973901 & 0.02287969 & 0.00313807 \\
\hline & 0.01369228 & -0.49987496 & 0.86606358 \\
\hline & 0.01864527 & -0.86578654 & -0.49992601 \\
\hline \multirow{3}{*}{ Nominal } & 0.99963924 & 0.02683525 & 0.00171706 \\
\hline & 0.01393145 & -0.48241207 & 0.87586855 \\
\hline & 0.02296312 & -0.87552865 & -0.48254672 \\
\hline \multirow{3}{*}{ Calibrated } & 0.99973669 & 0.02291913 & 0.00172049 \\
\hline & 0.01245480 & -0.49988111 & 0.86603981 \\
\hline & 0.01933960 & -0.86578634 & -0.49996905 \\
\hline
\end{tabular}

Since the rivet gun frame was designed to be perfectly aligned with the end-effector's frame with nominal angles of zeros, the calibrated values is the summation of the nominal values and the orientation errors presented in Table 6-10 above. As a result the calibrated angles are the same as the orientation errors.

The second trial of the orientation calibration was performed by increasing the number of the population size to 300 while maintaining the same number of 500 generations. It was observed that the best fitness value is the same as the previous trial and it stays constant as shown in Figure 6-11, even in the case of increasing the population size or the number of 
generations. The orientation errors obtained from the second calibration trial was tabulated in Table 6-11 below.

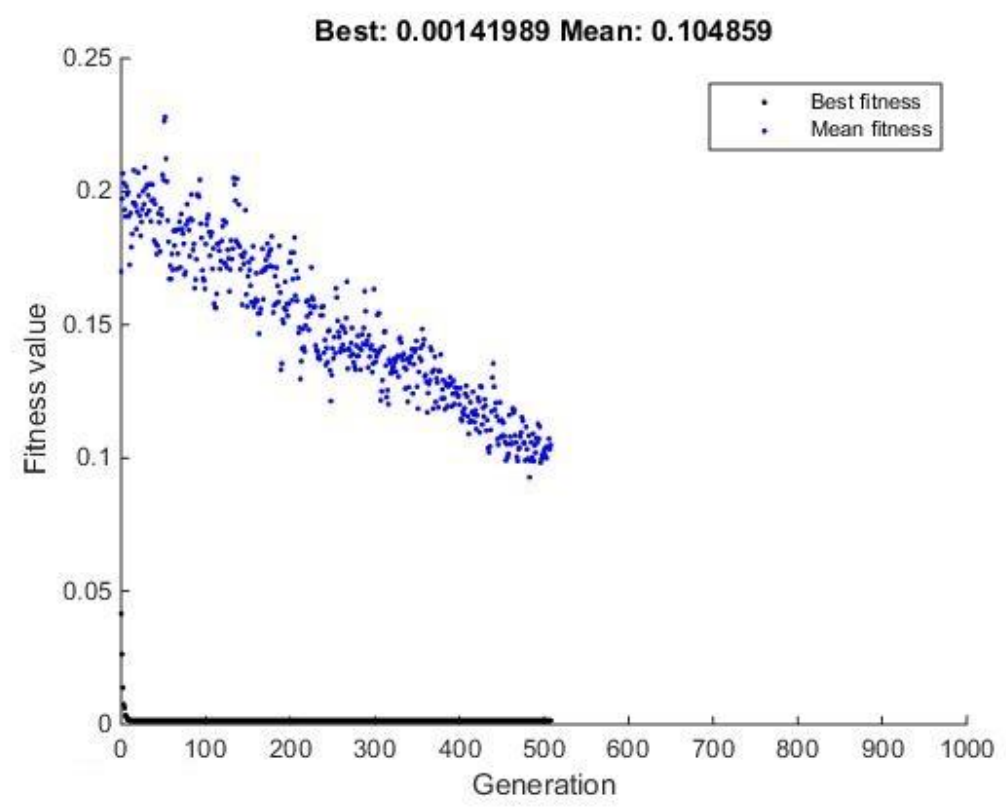

Figure 6-11: Orientation Only Calibration with 500 Generations and 300 Population Size

Table 6-11: Errors with 500 Generations and 300 Population Size

\begin{tabular}{|c|c|c|}
\hline $\boldsymbol{\delta} \mathbf{x}(\mathbf{d e g})$ & $\boldsymbol{\delta y} \mathbf{( d e g )}$ & $\boldsymbol{\delta z} \mathbf{( d e g})$ \\
\hline-1.14556673 & 0.03267025 & -1.53282557 \\
\hline
\end{tabular}

\subsection{Nonlinear Full Pose Calibration}

The final calibration process is the full pose calibration of the rivet gun. The full pose calibration was performed by setting the weight factor of the position parameters as well as the weight factor of the orientation parameters to one. This will include both the orientation and position parameters in the fitness function. 
Multiple trials were performed for obtaining the best full pose calibration performance. The GA parameters were set to 500 Generations and 100, 300 and 500 population sizes. The best fitness values corresponding to each of the population sizes are $0.00172163,0.00142031$ and 0.00141994 respectively. The results are shown in Figures 6-12, 6-13 and 6-14 below.

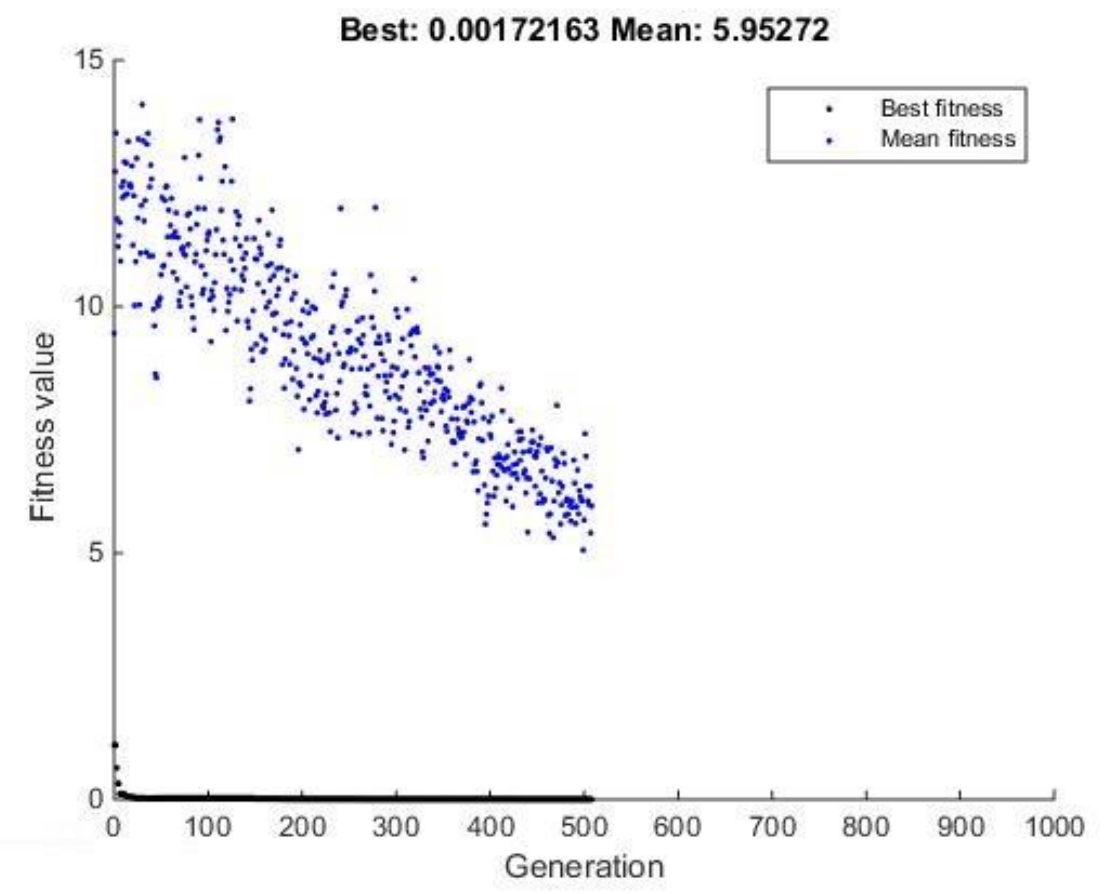

Figure 6-12: Full Pose Calibration with 500 Generations and 100 Population Size

The position and orientation errors as well as the calibrated rotation matrix and the position vector are tabulated in Tables 6-12 and 6-13.

Table 6-12: Position and Orientation Errors Based on 500 Generations and 100 Population Size

\begin{tabular}{|c|c|c|c|c|c|}
\hline$\Delta \mathbf{x}(\mathbf{m m})$ & $\Delta \mathbf{y}(\mathbf{m m})$ & $\Delta \mathbf{z}(\mathbf{m m})$ & $\boldsymbol{\delta x}(\mathbf{d e g})$ & $\boldsymbol{\delta y}(\mathbf{d e g})$ & $\boldsymbol{\delta z}(\mathbf{d e g})$ \\
\hline-0.70577135 & -0.51034633 & 0.00101015 & -1.11740481 & -0.03275695 & 0.24175341 \\
\hline
\end{tabular}


Table 6-13: Calibrated Rotation Matrix and Position Vector Based on 500 Generations and 100 Population size

\begin{tabular}{|c|c|c|c|c|}
\hline & \multicolumn{3}{|c|}{ Rotation Matrix } & Position Vector \\
\hline \multirow{3}{*}{ Calibrated } & 0.99974439 & 0.02259051 & 0.00158633 & 50.71444665 \\
\cline { 2 - 5 } & 0.01239659 & -0.49945512 & 0.86628639 & 331.20811731 \\
\cline { 2 - 5 } & 0.01899284 & -0.86604080 & -0.49954145 & -1980.23125971 \\
\hline
\end{tabular}

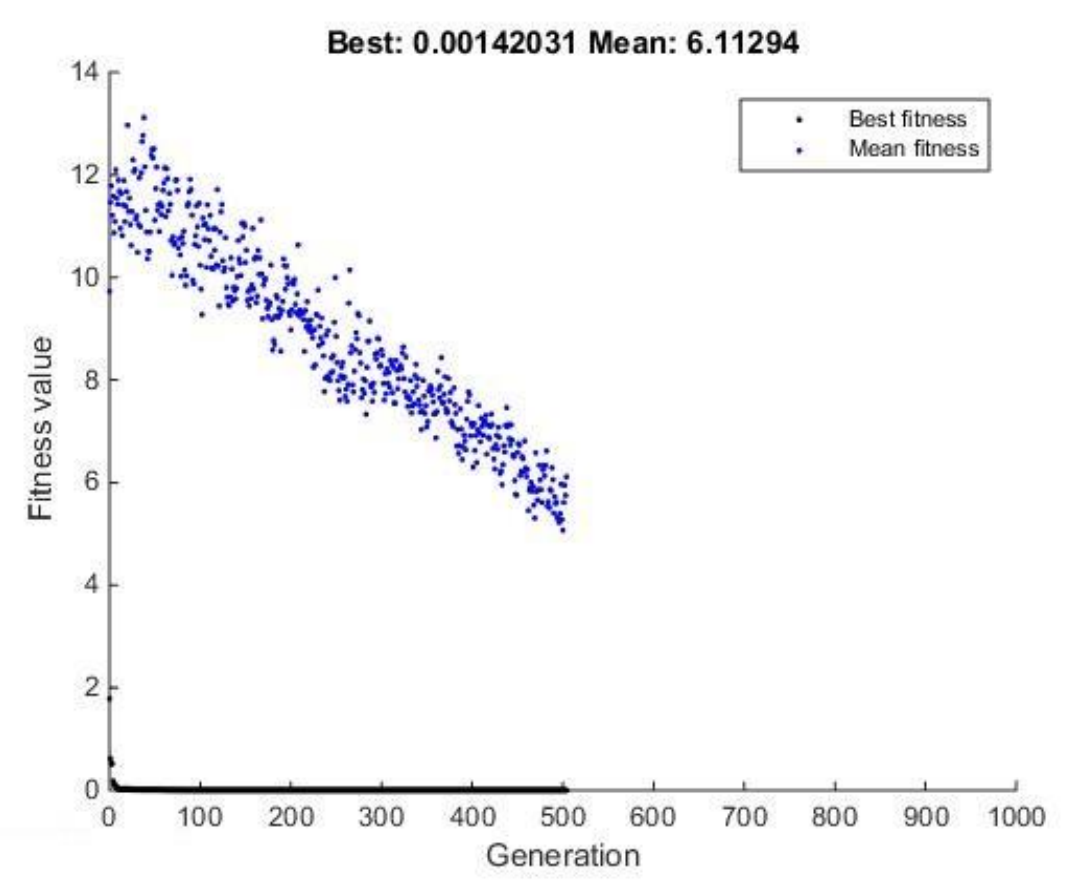

Figure 6-13: Full Pose Calibration with 500 Generations and 300 Population Size

Table 6-14: Position and Orientation Errors with 500 Generations and 300 Population Size

\begin{tabular}{|c|c|c|c|c|c|}
\hline$\Delta \mathbf{x}(\mathbf{m m})$ & $\Delta \mathbf{y}(\mathbf{m m})$ & $\Delta \mathbf{z}(\mathbf{m m})$ & $\boldsymbol{\delta x}(\mathbf{d e g})$ & $\boldsymbol{\delta y}(\mathbf{d e g})$ & $\boldsymbol{\delta z}(\mathbf{d e g})$ \\
\hline-0.70581548 & -0.51034168 & 0.00097945 & -1.14641395 & -0.02608559 & 0.22055166 \\
\hline
\end{tabular}


Table 6-15: Calibrated Rotation Matrix and Position Vector Based on 500 Generations and 300 Population Size

\begin{tabular}{|l|c|c|c|c|}
\hline & \multicolumn{3}{|c|}{ Rotation Matrix } & Position Vector \\
\hline \multirow{3}{*}{ Calibrated } & 0.99973581 & 0.02295725 & 0.00172156 & 50.71440262 \\
\cline { 2 - 5 } & 0.01247314 & -0.49988926 & 0.86603485 & 331.20808756 \\
\cline { 2 - 5 } & 0.01937304 & -0.86578062 & -0.49997766 & -1980.23124998 \\
\hline
\end{tabular}

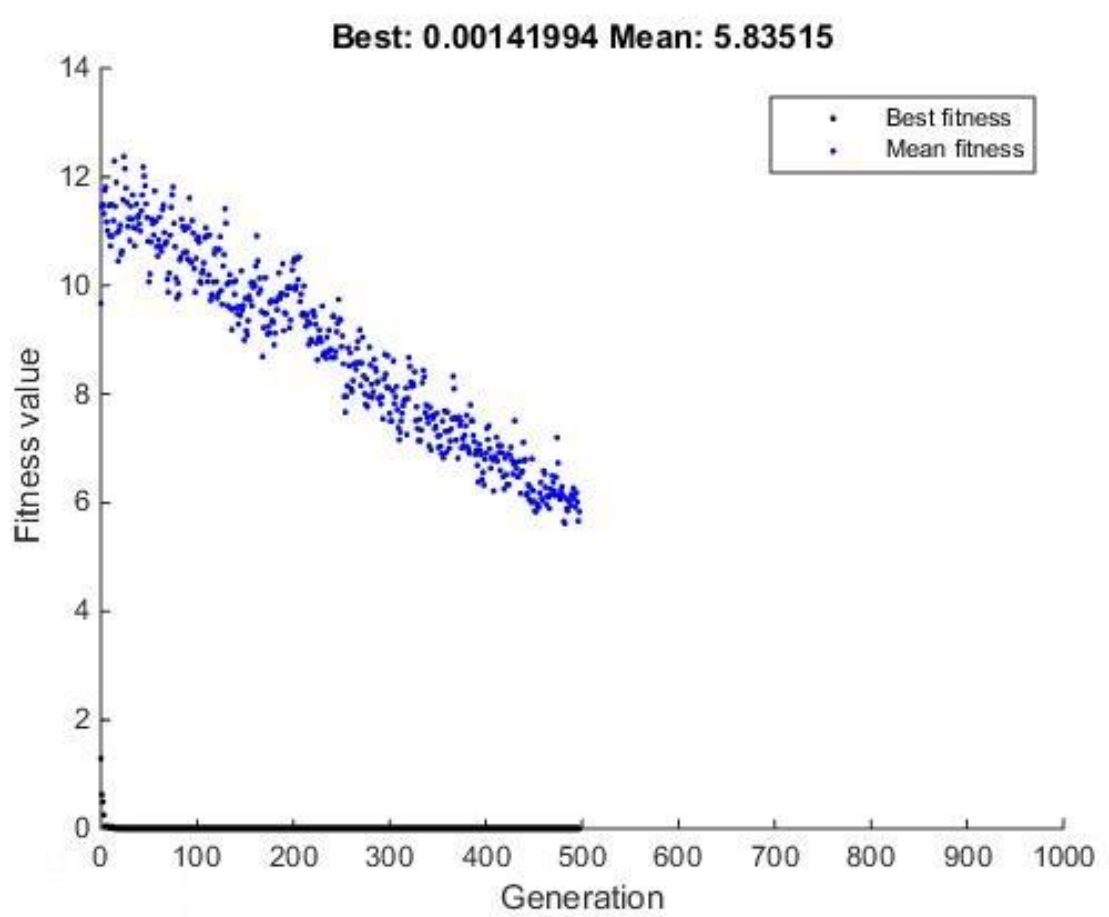

Figure 6-14: Full Pose Calibration with 500 Generations and 500 Population Size

Table 6-16: Position and Orientation Errors with 500 Generations and 500 Population Size

\begin{tabular}{|c|c|c|c|c|c|}
\hline$\Delta \mathbf{x}(\mathbf{m m})$ & $\boldsymbol{\Delta y}(\mathbf{m m})$ & $\boldsymbol{\Delta z}(\mathbf{m m})$ & $\boldsymbol{\delta x}(\mathbf{d e g})$ & $\boldsymbol{\delta y}(\mathbf{d e g})$ & $\boldsymbol{\delta} \mathbf{z}(\mathbf{d e g})$ \\
\hline-0.70581560 & -0.51034164 & 0.00097941 & -1.14692394 & -0.02608552 & 0.22104372 \\
\hline
\end{tabular}


Table 6-17: Calibrated Rotation Matrix and Position Vector Based on 500 Generations and 500 Population Size

\begin{tabular}{|c|c|c|c|c|}
\hline & \multicolumn{3}{|c|}{ Rotation Matrix } & Position Vector \\
\hline \multirow{3}{*}{ Calibrated } & 0.99973601 & 0.02294865 & 0.00172159 & 50.71440250 \\
\cline { 2 - 5 } & 0.01246900 & -0.49989707 & 0.86603040 & 331.20808750 \\
\cline { 2 - 5 } & 0.01936553 & -0.86577634 & -0.49998537 & -1980.23125000 \\
\hline
\end{tabular}

It was observed that the best fitness does not decrease below 0.00141994 by increasing the population sizes above 500 neither by increasing the number of generations above 500 . 


\section{CHAPTER 7: CONCLUSION, CONTRIBUTIONS AND FUTURE WORK}

\subsection{Conclusion}

Robotic Tooling Calibration is a very critical step in the automation process of manufacturing especially during the tasks that require high level of accuracy. Different tool calibration methods were investigated and developed in this thesis. First, the system required for the calibration method was designed for considering more than one calibration approach. The first formulation considered for the tooling calibration is the linear formulation. The linear formulation was extensively studied by developing two approaches; the first is based on the conventional calibration using least squares, while the second is based on self-calibration formulation. The linear method based on self-calibration was analyzed and proven to be an unsuccessful approach for tool calibration due to Jacobian rank deficiency. The conventional calibration formulation was analyzed and implemented on the experimental data. The second formulation for determining the errors as well as calibrating the tool is the nonlinear approach. A nonlinear formulation was developed in this thesis using genetic algorithm as a search method for determining the tool errors. The method was simulated and the limitations regarding the endeffector position and the tool orientation estimation were determined. The method was applied on the experimental data obtained from the actual robot system set-up and the errors were identified and the tool geometric parameters were calibrated.

\subsection{Contributions}

The contributions that were provided in this thesis could be summarized in the following points, 
1. A robotic tooling calibration approach was developed based on a nonlinear approach using genetic algorithm. The developed method is robust and simple to apply in a manufacturing environment. The method uses an off-line error measurement approach which does not require any robot movements and reduces the down time of the manufacturing line.

2. The linear formulation based on the self-calibration formulation was analyzed. The root causes of the ineffectiveness of the linear formulation based on self-calibration algorithm was determined and discussed. The causes are traced back to the Jacobian rank deficiency.

3. The Linear formulation based on the conventional calibration, using least squares, was analyzed and implemented on the experimental data.

4. A tool orientation estimation method was developed in this thesis to accurately measure the tool orientation. The method was developed by formulating a cylinder fitting algorithm which uses genetic algorithm for determining all the cylinder's parameters.

\subsection{Future Work}

Robotic tooling calibration is a crucial topic which requires a lot of research for covering all of its different aspects. Several recommendations are provided below based on the research conducted in this thesis for future work. 
First, the tool geometric errors obtained from both the linear formulation approach as well as the nonlinear formulation should be implemented for tool calibration. In other words, the errors determined should be inserted into the robot's controller. Multiple tests have to be conducted to determine whether the robot can be commanded to successfully insert a rivet into a predetermined hole.

Second, the robot was considered to be calibrated before tool calibration takes place. However, the effects of the tool weight on the accuracy of the robotic manipulator are eminent and should be extensively studied. A tool calibration model should be developed to account for the robot manipulator errors due to the tool weight.

Third, the method developed in this thesis could be expanded to include the calibration of the robotic manipulator as well. By locating the tool tip pose and the vision system location with respect to the base frame, a formulation could be developed for including the robot geometric parameters errors. Also a study has to be conducted to determine the effects and contribution of the robot geometric errors to the tool tip errors.

Fourth, the orientation estimation of different tool shapes must be considered. Different shapes fitting techniques must be developed for determining the location of different types of tools. Also, a laser scanner measurement tool is considered to be more appropriate for determining more complex shapes. For accurately determining the shape of a cylinder, around 300 points were required. Using a laser scanner could significantly provide a lot of points in a short period of time. 


\section{LIST OF APPENDICES}

\section{APPENDIX A-PLANE FITTING}

The equation of a plane could be described using the following equations,

$$
A x+B y+C z+D=0
$$

By reordering the terms,

$$
z=E x+F y+G
$$

Minimizing the distance between the plane and the points using the following equations,

$$
\begin{gathered}
e=z_{i}-(E x+F y+G) \\
\varepsilon=\sum\left(z_{i}-(E x+F y+G)\right)^{2}
\end{gathered}
$$

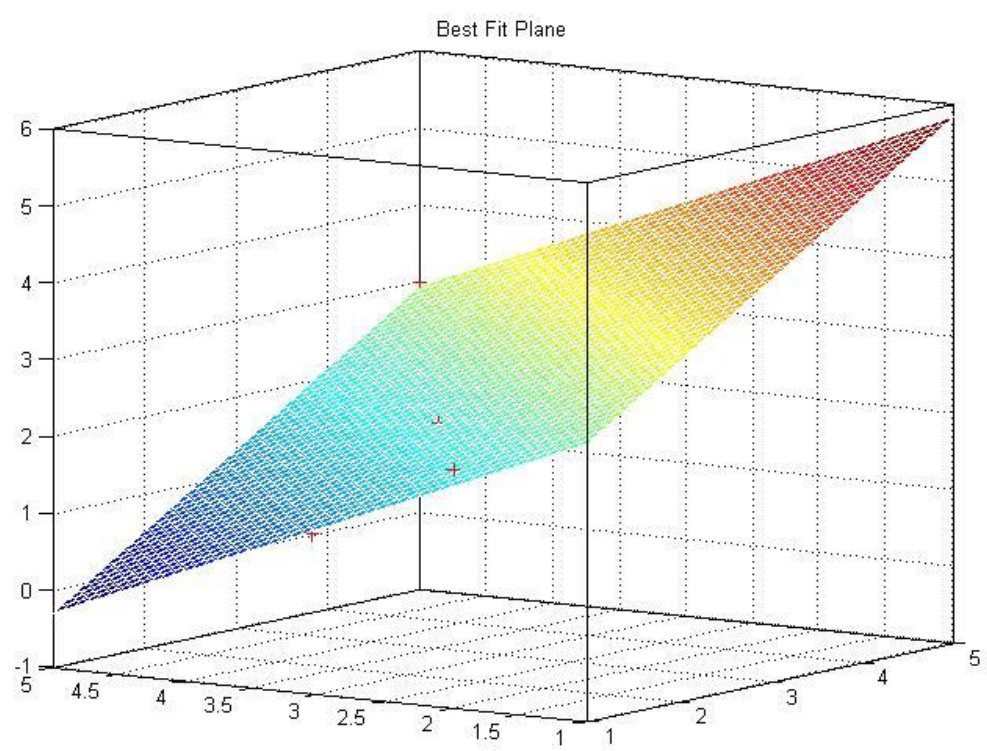

Figure A-1: Best Fit Plane Generated for Multiple Points 


\section{APPENDIX B - KASA METHOD}

A circle in a 2D plane could be expressed using the following equation,

$$
\left(x-x_{c}\right)^{2}+\left(y-y_{c}\right)^{2}=r^{2}
$$

By minimizing the errors between the points and the edge of the circle given the following equation,

$$
f_{i}=\left(x_{i}-x_{c}\right)^{2}+\left(y_{i}-y_{c}\right)^{2}-r^{2}
$$

By taking the derivative of the function above and linearizing the parameters using the following,

$$
B=-2 x_{c}, \quad C=-2 y_{c}, \quad D=x_{c}{ }^{2}+y_{c}{ }^{2}-r^{2}
$$

By expanding Equation A.6 and substituting the values in Equation A.7,

$$
\begin{gathered}
f_{i}=x_{i}^{2}+x_{c}^{2}-2 x_{i} x_{c}+y_{i}^{2}+y_{c}^{2}-2 y_{i} y_{c}-r^{2} \\
f_{i}=\left(x_{i}^{2}+y_{i}^{2}\right)+B x_{i}+C y_{i}+\mathrm{D} \\
f_{i}=z_{i}+B x_{i}+C y_{i}+D \\
\min \left(\sum f_{i}\right)
\end{gathered}
$$

Equation (A.B.7) is done by taking the differentiation with respect to B, C and D and then solving the system of equations. The parameters of the circle could be easily obtained using the following equations,

$$
x_{c}=-\frac{B}{2}, \quad y_{c}=-\frac{C}{2}, \quad r=\frac{\sqrt{B^{2}+C^{2}-4 D}}{2}
$$




\section{APPENDIX C-CYLINDER EQUATIONS}

A cylinder that has its axis aligned with the Z-axis of a frame 2 could be expressed using the following equations,

$$
\begin{gathered}
X=r \cos (\gamma) \\
Y=r \sin (\gamma) \\
Z=Z
\end{gathered}
$$

For expressing a general orientation cylinder the following sequence of rotations must be applied as shown in Figure A-2 and described in the following equations,

$$
R_{2}^{1}=R_{Z} R_{Y}
$$

where

$$
\begin{gathered}
\boldsymbol{R}_{\boldsymbol{Z}}(\theta)=\left[\begin{array}{ccc}
\operatorname{Cos} \theta & -\operatorname{Sin} \theta & 0 \\
\operatorname{Sin} \theta & \operatorname{Cos} \theta & 0 \\
0 & 0 & 1
\end{array}\right] \\
\boldsymbol{R}_{\boldsymbol{Y}}(\varphi)=\left[\begin{array}{ccc}
\operatorname{Cos} \varphi & 0 & \operatorname{Sin} \varphi \\
0 & 1 & 0 \\
-\operatorname{Sin} \varphi & 0 & \operatorname{Cos} \varphi
\end{array}\right] \\
\boldsymbol{R}_{2}^{1}=\boldsymbol{R}_{\boldsymbol{Z}}(\theta) \boldsymbol{R}_{\boldsymbol{Y}}(\varphi)=\left[\begin{array}{ccc}
\operatorname{C\theta S} \varphi & -S \theta & \operatorname{C\theta S} \varphi \\
\operatorname{S\theta C} \varphi & \operatorname{C\theta } & \operatorname{S\theta S} \varphi \\
-S \varphi & 0 & C \varphi
\end{array}\right]
\end{gathered}
$$

The cylinder equations expressed above could be transformed using the following equation,

$$
\left[\begin{array}{l}
A \\
B \\
C
\end{array}\right]=\boldsymbol{R}_{1}^{2}\left[\begin{array}{l}
X \\
Y \\
Z
\end{array}\right]
$$




$$
\begin{gathered}
{\left[\begin{array}{l}
A \\
B \\
C
\end{array}\right]=\left[\begin{array}{ccc}
C \theta S \varphi & S \theta C \varphi & -S \varphi \\
-S \theta & C \theta & 0 \\
C \theta S \varphi & S \theta S \varphi & C \varphi
\end{array}\right]\left[\begin{array}{l}
X \\
Y \\
Z
\end{array}\right]} \\
A=X \operatorname{Cos}(\theta) \operatorname{Sin}(\varphi)+Y \sin (\theta) \operatorname{Cos}(\varphi)-Z \operatorname{Sin}(\varphi) \\
B=-X \operatorname{Sin}(\theta)-Y \operatorname{Cos}(\theta) \\
C=X \operatorname{Cos}(\theta) \operatorname{Sin}(\varphi)+Y \operatorname{Sin}(\theta) \operatorname{Sin}(\varphi)+Z \operatorname{Cos}(\varphi)
\end{gathered}
$$
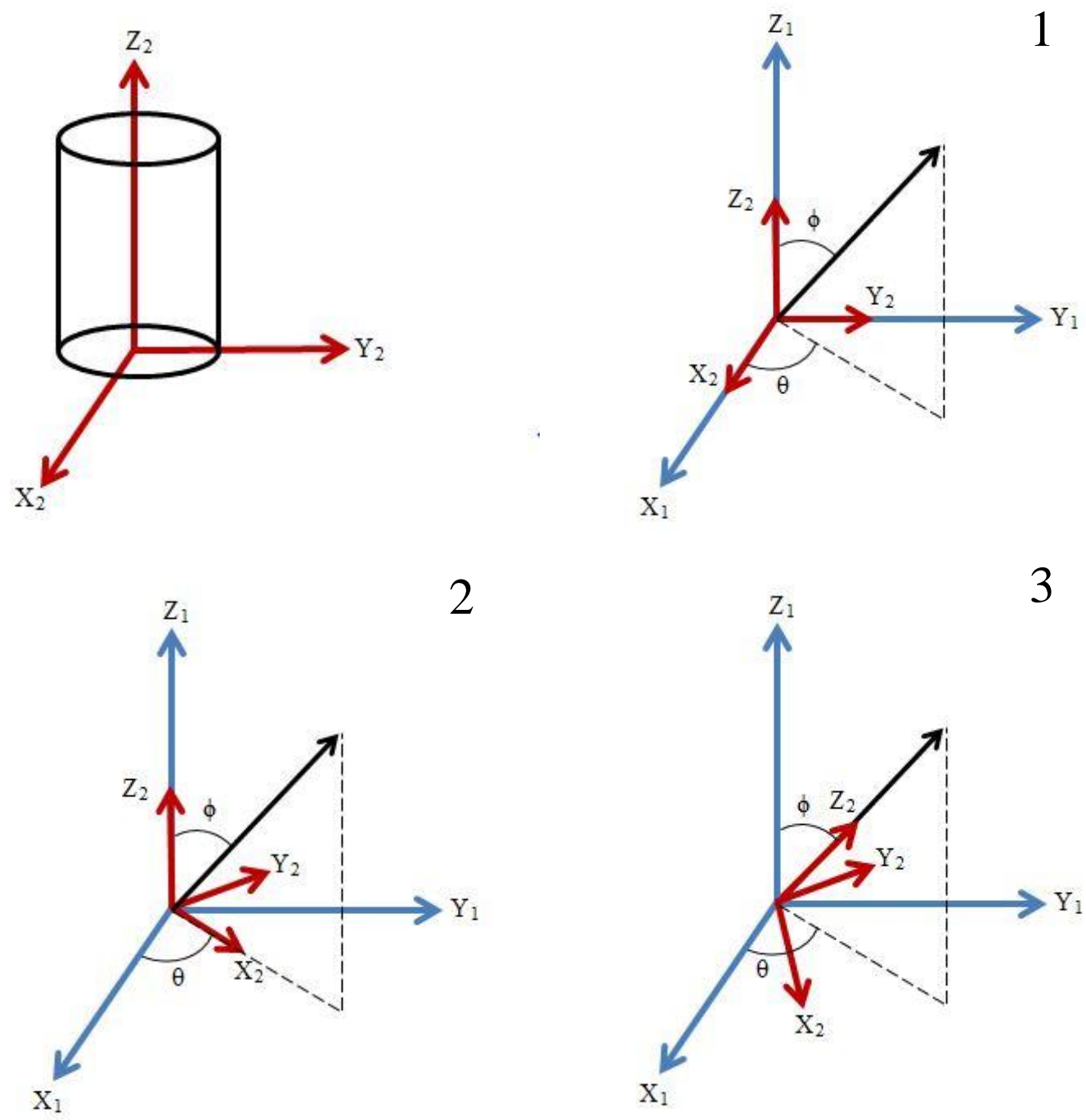

Figure A-2: Cylinder Rotation Sequence 


\section{APPENDIX D-ROBOT END-EFFECTOR IN BASE FRAME}

The robot's end-effector pose with respect to the base frame could be easily obtained from the robot's controller as presented in Table A-1 below. Using the parameters obtained from the controller, the homogeneous transformation from the end-effector to the base frame could be calculated as presented in Table A-2 below.

Table A-1: End-Effector Parameters with respect to the Base Frame

\begin{tabular}{|c|c|c|c|c|c|}
\hline $\mathbf{X}$ & $\mathbf{Y}$ & $\mathbf{Z}$ & $\mathbf{E Z}$ & $\mathbf{E Y}$ & $\mathbf{E X}$ \\
\hline 1150.8 & 28.7 & 1324.9 & 0 & 90 & 0 \\
\hline
\end{tabular}

Table A-2: Rotation Matrix and Position Vector of the End-Effector with respect to the Base Frame

\begin{tabular}{|c|c|c|c|}
\hline \multicolumn{3}{|c|}{ Rotation Matrix } & $\begin{array}{c}\text { Position Vector } \\
\text { (mm) }\end{array}$ \\
\hline 0 & 1 & 0 & 1150.8 \\
\hline 0 & 0 & 1 & 28.7 \\
\hline-1 & 0 & 0 & 1324.9 \\
\hline
\end{tabular}

\section{APPENDIX E-JACOBIAN MATRIX}

The calculated Jacobian Matrix $\boldsymbol{J}_{E}$,

$$
J_{E}=\left[\begin{array}{cccccc}
1 & 0 & 0 & 0 & 370.5 & 0 \\
0 & 1 & 0 & -370.5 & 0 & 0 \\
0 & 0 & 1 & 0 & 0 & 0 \\
0 & 0 & 0 & 1 & 0 & 0 \\
0 & 0 & 0 & 0 & 1 & 0 \\
0 & 0 & 0 & 0 & 0 & 1
\end{array}\right]
$$




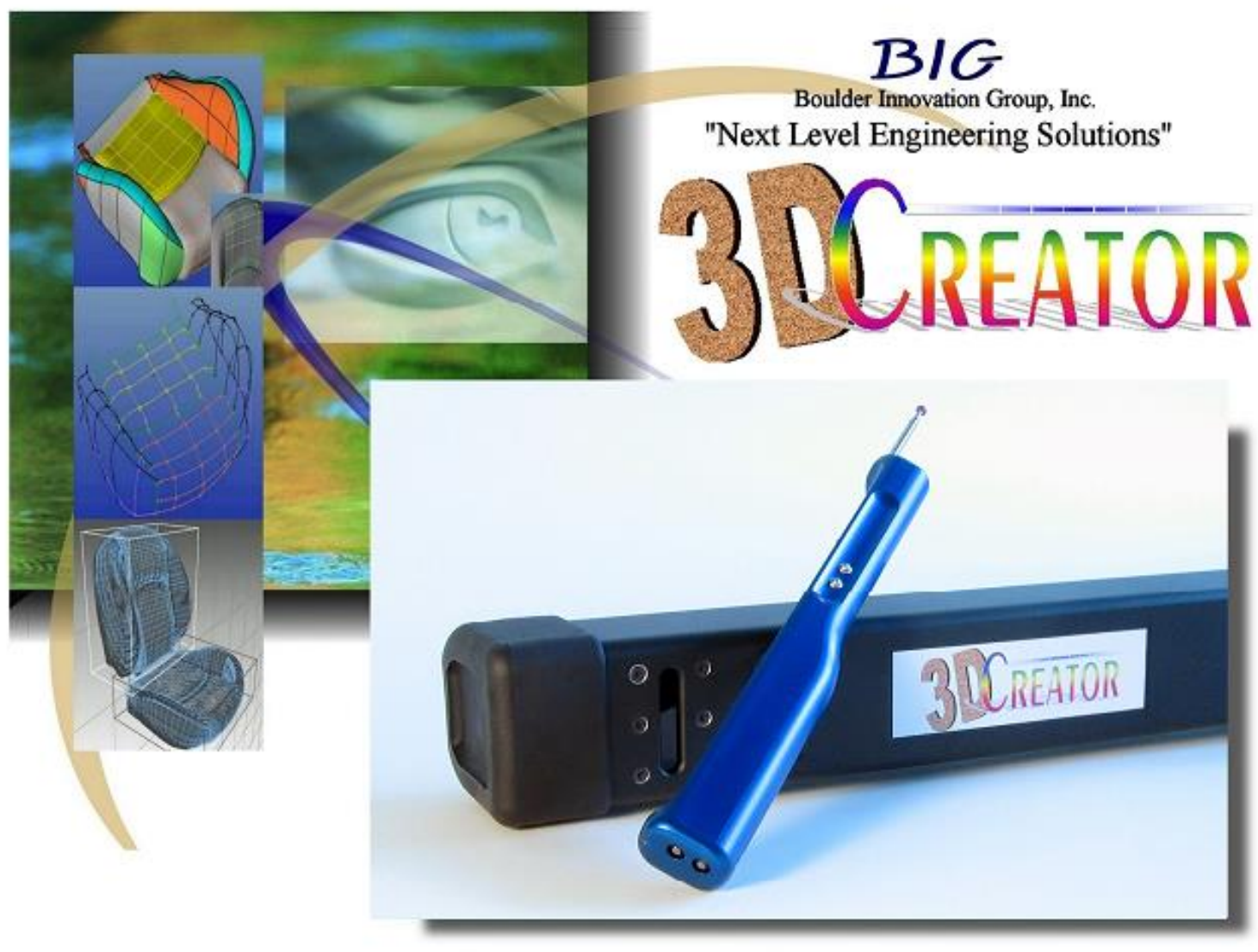

Enables precise 3-Dimensional measurement

Allows contour, seam and surface capture

The ultimate in a portable CMM

Time saved will pay for itself

Provides real-time tracking of instruments providing $X, Y, Z$ and vector information

The productivity enhancer

\section{The Path to Productivity}

\section{Superior 3-D digitizing for the ultimate in object capture}

The affordable 3D-Creator Optical Digitizer provides the best value proposition in the digitizing market today. Offering the utmost in flexibility, without mechanical arms, and the empowerment to concentrate on the task at hand: Being Productive. The 3D-Creator's free-hand wireless operation is unique in its ability to measure object details without mechanical restrictions. The 3D-Creator is ideal for measuring objects big and small. It is well suited for parts inspection, general digitizing and reverse engineering. Flexibility in measuring makes the 3D-Creator suitable for applications in automotive, boating, 2D pattern cutting and precision parts inspection.

\section{Innovative engineering from a global technology leader}

The time and money saving 3D-Creator features wireless instrumentation. Boulder Innovation Group, Inc. provides some of the world's most advanced technologies for accuracy and versatility of application. Truly, Next level engineering solutions.

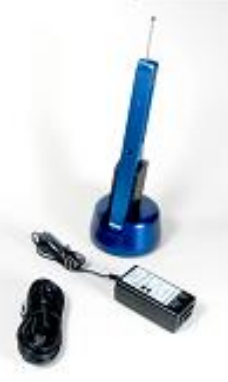




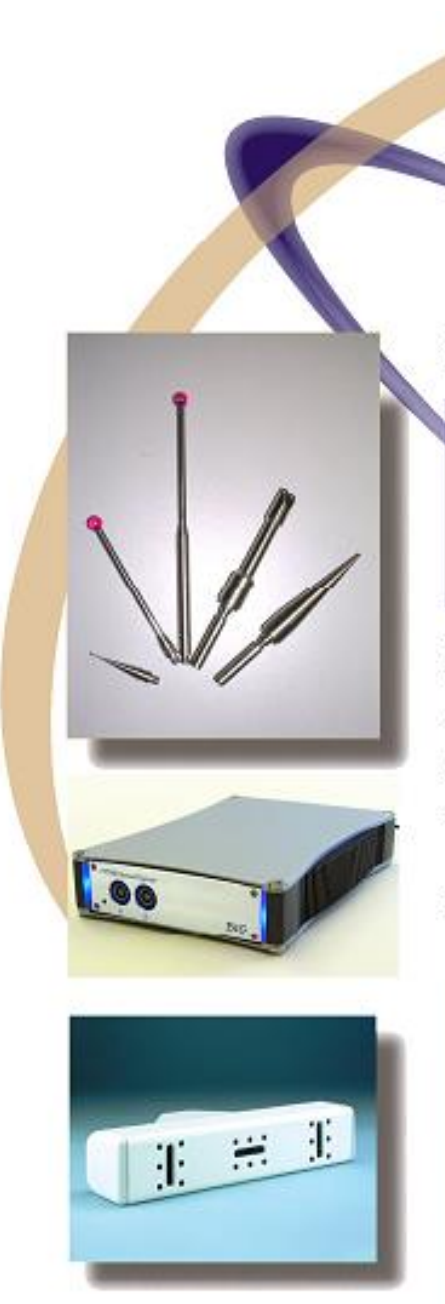

\section{D Creator System Technical Data}

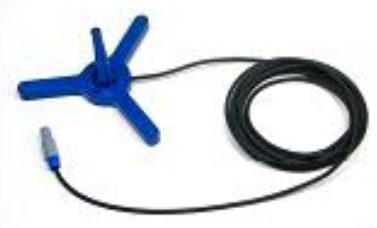

Tracking:

Tracks 1 to 256 LEDs in the field at the same time

Data output: $x, y, z$ coordinates and $u, v$ vectors via serial ASCII and binary (user-configurable format)

Throughput:

Up to $300 \mathrm{hz}$

\section{D Creator Includes:}

- Control Unit w/ Power Supply

$.800 \mathrm{~mm}$ Sensor Array

- 15 Ft Sensor Cable

- Carrying Case

- Wireless Probe

- Probe Charger Cradle

- USB to Serial Adapter

-Serial Cable
- 2 Probe Tips

- $140 \mathrm{~mm}$ DRF

- Tripod w/ Head

\section{D Creator Accuracy Data}

\section{$B / G$}

Boulder Innovation Group, Inc. 4824 Sterling Drive

Boulder, CO 80301, USA

Telephone: (303) 447-0248

Fax: (303) 447-3905

www.boulderinnovators.com

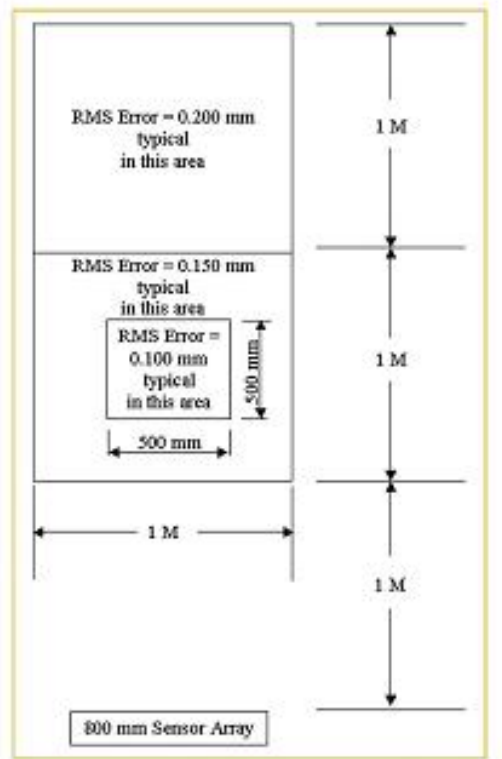

Boulder Innovation Group, Inc. All Rights Reserved. P/N 140274 Rev. F
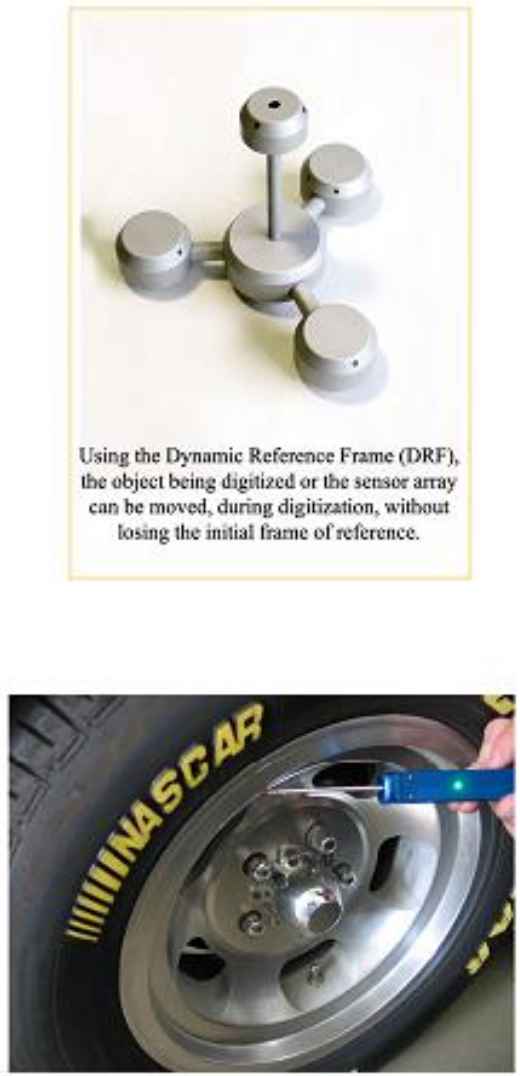


\section{REFERENCES}

[1] Wallén, J. (2008). The history of the industrial robot. Technical Report from Automatic Control at Linkopings University, Linkoping, Sweden.

[2] Craig, J. J. (2005). Introduction to robotics: mechanics and control Vol. 3. Upper Saddle River: Pearson Prentice Hall.

[3] Lin, Y. (2008). Robot calibration based on nonlinear formulation for modular reconfigurable robots (MRRS) (Master's Thesis). Ryerson University. School of Graduate Studies. Program of Mechanical Engineering.

[4] Schneider, U., Ansaloni, M., Drust, M., Leali, F., \& Verl, A. (2013). Experimental Investigation of Sources of Error in Robot Machining. In Robotics in Smart Manufacturing (pp. 14-26). Berlin: Springer.

[5] Khalil, W., \& Dombre, E. (2004). Modeling, identification and control of robots. Oxford: Butterworth-Heinemann.

[6] Everett, L., Driels, M., \& Mooring, B. (1987). Kinematic modelling for robot calibration. In IEEE International Conference on Robotics and Automation. Proceedings. Vol. 4, pp. $183-189$.

[7] Denavit, J. (1955). A kinematic notation for lower-pair mechanisms based on matrices. Trans. of the ASME. Journal of Applied Mechanics, 22, 215-221.

[8] Paul, R. P., \& Shimano, B. (1979). Kinematic control equations for simple manipulators. In IEEE Conference on Decision and Control including the 17th Symposium on Adaptive Processes, pp. 1398-1406.

[9] Ibarra, R., \& Perreira, N. D. (1985). Determination of Linkage Parameter and Pair Variable Errors in Open Chain Kinematic Linkages Using a Minimal Set of Position 
Measurement Data. ASME Paper, 85-DET, 51.

[10] Wu, C. (1984). A Kinematic CAD tool for the design and control of a robot manipulator. The International Journal of Robotics Research, 3(1), 58-67.

[11] Wu, C. H. (1983). The kinematic error model for the design of robot manipulator. In IEEE on American Control Conference, pp. 497-502.

[12] Zhen, H. (1985). Error analysis of robot manipulators and error transmission functions. Proc. of the 15th ISIR, Tokyo, pp. 873-878.

[13] Payannet, D., Aldon, M. J., \& Liegeois, A. (1985). Identification and compensation of mechanical errors for industrial robots. Proc.15th Int.Symp.on Industrial Robots, 857864.

[14] Mooring, B. W. (1983). The effect of joint axis misalignment on robot positioning accuracy. Proceedings of the ASME International Computers in Engineering Conference, 151-156.

[15] Hayati, S. A. (1983). Robot arm geometric link parameter estimation. In The 22nd IEEE Conference on Decision and Control, 1983. Vol. 22, pp. 1477-1483.

[16] Mooring, B. W., Roth, Z. S., \& Driels, M. R. (1991). Fundamentals of manipulator calibration. Toronto: John Wiley \& Sons, Inc.

[17] Hayati, S. A., \& Mirmirani, M. (1984). A software for robot geometry parameter estimation. In Robots West Conference, Anaheim, California.

[18] Stone, H., Sanderson, A., \& Neuman, C. (1986). Arm signature identification. In IEEE International Conference on Robotics and Automation. Proceedings. Vol. 3, pp. 41-48.

[19] Hsu, T. W. (1987). Robot accuracy improvement through kinematic parameter identification (Doctoral dissertation). ProQuest, UMI Dissertation Publishing. 
[20] Paul, R. P. (1981). Robot manipulators: mathematics, programming, and control: the computer control of robot manipulators. Cambridge: MIT Press.

[21] Whitney, D. E., Lozinski, C. A., \& Rourke, J. M. (1986). Industrial robot forward calibration method and results. Journal of Dynamic Systems, Measurement, and Control, $108(1), 1-8$.

[22] Hollerbach, J. M. (1989). A survey of kinematic calibration. In The robotics review 1, pp. 207-242. Cambridge: MIT Press.

[23] Chen, J., \& Chao, L.-M. (1987). Positioning error analysis for robot manipulators with all rotary joints. IEEE Journal on Robotics and Automation, 3(6), 539-545.

[24] Gatla, C. S., Lumia, R., Wood, J., \& Starr, G. (2007). An automated method to calibrate industrial robots using a virtual closed kinematic chain. Robotics, IEEE Transactions on Robotics, 23(6), 1105-1116.

[25] Lightcap, C., Hamner, S., Schmitz, T., \& Banks, S. (2008). Improved positioning accuracy of the PA10-6CE robot with geometric and flexibility calibration. IEEE Transactions on Robotics, 22(2), 452-456.

[26] Yurttagul, B. (2010). Kinematic calibration of industrial robots using full pose measurements and optimal pose selection. Ankara, Turkey: METU.

[27] Joubair, A., Nubiola, A., \& Bonev, I. (2013). Calibration efficiency analysis based on five observability indices and two calibration models for a six-axis industrial robot. $S A E$ International Journal of Aerospace, 6(1), 161-168.

[28] Doebelin, E. O. (2004). Measurement systems: application and design. Boston: McGraw-Hill.

[29] Gong, C., Yuan, J., \& Ni, J. (2000). Nongeometric error identification and compensation 
for robotic system by inverse calibration. International Journal of Machine Tools and Manufacture, 40(14), 2119-2137.

[30] Majarena, A. C., Santolaria, J., Samper, D., \& Aguilar, J. J. (2011). Modelling and calibration of parallel mechanisms using linear optical sensors and a coordinate measuring machine. Measurement Science and Technology, 22(10), 105101.

[31] Meng, G., Tiemin, L., \& Wensheng, Y. (2003). Calibration method and experiment of Stewart platform using a laser tracker. In IEEE International Conference on Systems, Man and Cybernetics, Vol. 3, pp. 2797-2802.

[32] TP200 co-ordinate measuring machines, photograph (2012), Retrieved 29 May 2015, http://www.th.all.biz/img/th/catalog/32617.jpeg.

[33] Mooring, B. W., \& Padavala, S. S. (1989). The effect of kinematic model complexity on manipulator accuracy. In IEEE International Conference on Robotics and Automation. Proceedings, pp. 593-598.

[34] Zhuang, H., Wang, L. K., \& Roth, Z. S. (1993). Error-model-based robot calibration using a modified CPC model. Robotics and Computer Integrated Manufacturing, 10(4), 287-299.

[35] Cooper, M. A. R. (1982). Modern theodolites and levels. Oxford: BSP Professional Books.

[36] Remy, S., Dhome, M., Lavest, J. M., \& Daucher, N. (1997). Hand-eye calibration. In Proceedings of the IEEE/RSJ International Conference on Intelligent Robots and Systems, Vol. 2, pp. 1057-1065.

[37] Motta, J. M. S. T., Carvalho, G. C. de, \& McMaster, R. S. (2001). Robot calibration using a 3D vision-based measurement system with a single camera. Robotics and Computer 
Integrated Manufacturing, 17(6), 487-497.

[38] Visual servoing: uncalibrated hand-eye coordination through differential visual feedback, photograph, viewed 20 May 2015, https://www.cs.rochester.edu/u/jag/PercAct/dvfb.html.

[39] Pathre, U. S., \& Driels, M. R. (1990). Simulation experiments in parameter identification for robot calibration. The International Journal of Advanced Manufacturing Technology, $5(1), 13-33$.

[40] Siciliano, B., \& Khatib, O. (2008). Springer handbook of robotics. Stanford: Springer Science \& Business Media.

[41] Levenberg, K. (1944). A method for the solution of certain non-linear problems in least squares. Quarterly Journal of Applied Mathmatics, II (2), 164-168.

[42] Marquardt, D. W. (1963). An algorithm for least-squares estimation of nonlinear parameters. Journal of the Society for Industrial and Applied Mathematics, 11(2), 431441.

[43] Chao, L. M., \& Yang, J. C. S. (1986). Development and implementation of a kinematic parameter identification technique to improve the positioning accuracy of robots. Society of Manufacturing Engineers.

[44] Judd, R. P., \& Knasinski, A. B. (1990). A technique to calibrate industrial robots with experimental verification. IEEE Transactions on Robotics and Automation, 6(1), 20-30.

[45] Gan, Y., \& Dai, X. (2011). Base frame calibration for coordinated industrial robots. Robotics and Autonomous Systems, 59(7), 563-570.

[46] Wang, H., Shen, S., \& Lu, X. (2012). A screw axis identification method for serial robot calibration based on the POE model. Industrial Robot: An International Journal, 39(2), 
146-153.

[47] Lin, Y., Xi, F., Mohamed, R., \& Tu, X. W. (2010). Calibration of modular reconfigurable robots based on a hybrid search method. Journal of manufacturing science and engineering, 132(6), 061002.

[48] Zhuang, H., Wang, K., \& Roth, Z. S. (1994). Optimal selection of measurement configurations for robot calibration using simulated annealing. In IEEE International Conference on Robotics and Automation, pp. 393-398.

[49] Sun, Y., \& Hollerbach, J. M. (2008). Observability index selection for robot calibration. In IEEE International Conference on Robotics and Automation, pp. 831-836.

[50] Driels, M. R., \& Pathre, U. S. (1990). Significance of observation strategy on the design of robot calibration experiments. Journal of Robotic Systems, 7(2), 197-223.

[51] Watanabe, A., Sakakibara, S., Ban, K., Yamada, M., Shen, G., \& Arai, T. (2006). A Kinematic Calibration Method for Industrial Robots Using Autonomous Visual Measurement. CIRP Annals - Manufacturing Technology, 55(1), 1-6.

[52] Khalil, W., Besnard, S., \& Lemoine, P. (2000). Comparison study of the geometric parameters calibration methods. International Journal of Robotics and Automation, 15(2), pp-56.

[53] Gong, C., Yuan, J., \& Ni, J. (2000). A Self-Calibration Method for Robotic Measurement System. Journal of Manufacturing Science and Engineering, 122(1), 174.

[54] Zhuang, H., Motaghedi, S. H., \& Roth, Z. S. (1999). Robot calibration with planar constraints. In IEEE International Conference on Robotics and Automation. Vol. 1, pp. 805-810.

[55] Dornaika, F., \& Horaud, R. (1998). Simultaneous robot-world and hand-eye calibration. 
IEEE Transactions on Robotics and Automation, 14(4), 617-622.

[56] Zhan, Q., \& Wang, X. (2012). Hand-eye calibration and positioning for a robot drilling system. The International Journal of Advanced Manufacturing Technology, 61(5), 691701.

[57] Wang, H., Fan, X., \& Lu, X. (2013). Application of a hand-eye self-calibration technique in robot vision. In Control and Decision Conference (CCDC), 25th Chinese on pp. 37653769.

[58] Axelrod, B., \& Huang, W. H. (2012). Improving hand-eye calibration for robotic grasping and manipulation. In IEEE International Conference on Technologies for Practical Robot Applications (TePRA), 2012 pp. 121-126.

[59] Hallenberg, J. (2007). Robot tool center point calibration using computer vision (Master's Thesis). Linköping, Sweden: University of Linkoping.

[60] Ayadi, A., Nicolau, S., Bayle, B., Graebling, P., \& Gangloff, J. (2007). Fully automatic needle calibration for robotic-assisted puncture on small animals. In IEEE/NIH on Life Science Systems and Applications Workshop, pp. 85-88.

[61] Cheng, F. S. (2007). The method of recovering robot TCP positions in industrial robot application programs. In International Conference on Mechatronics and Automation. pp. 805-810.

[62] Shiu, Y. C., \& Ahmad, S. (1989). Calibration of wrist-mounted robotic sensors by solving homogeneous transform equations of the form $\mathrm{AX}=\mathrm{XB}$. IEEE Transactions on Robotics and Automation, 5(1), 16-29.

[63] Park, F. C., \& Martin, B. J. (1994). Robot sensor calibration: solving AX=XB on the Euclidean group. IEEE Transactions on Robotics and Automation, 10(5), 717-721. 
[64] Zuang, H., \& Shiu, Y. C. (1993). A noise-tolerant algorithm for robotic hand-eye calibration with or without sensor orientation measurement. IEEE Transactions on Systems, Man, and Cybernetics, 23(4), 1168-1175.

[65] Daniilidis, K. (1999). Hand-eye calibration using dual quaternions. The International Journal of Robotics Research, 18(3), 286-298.

[66] Helal, M., Xi, F., \& Lin, Y. (2013). Robotic Tooling Self-Calibration. SAE Technical Paper. (No. 2013-01-2118).

[67] Chernov, N. (2010). Circular and linear regression: fitting circles and lines by least squares. Florida: CRC Press.

[68] Bullock, R., 2006. Least-Squares Circle Fit. Retrieved 29 May 2015, http://www.dtcenter.org/met/users/docs/write_ups/circle_fit.pdf.

[69] Xi, F., Lin, Y., Dakdouk, D., Helal, M., \& East, B. (2013). Patent No. WO 2013152440 A1. Canada.

[70] Holland, J. H. (1975). Adaptation in natural and artificial systems: an introductory analysis with applications to biology, control, and artificial intelligence. Oxford, England: U Michigan Press.

[71] McMaster, R., \& Ribeiro, F. M. (1994). Cell calibration and robot tracking. In Next Steps for Industrial Robotics, IEE Colloquium on pp. 3-1. 